\author{
Raúl Chiu Nazaralá
}

\title{
Análisis y desarrollo de observador empleando LMI aplicado a bioprocesos
}

26 de noviembre de 2009 



\section{Resumen}

El desarrollo de observadores para la estimación de variables y parámetros en un proceso biotecnológico es el tema central del presente trabajo. Se aborda así el desarrollo de un observador de estados que considera en su diseño las incertidumbres presentes en el modelado del sistema, definidas estas dentro de un politopo cuyos vértices son los límites de operación del sistema.

Este desarrollo se presenta en dos vertientes. Una es la aplicación del concepto a un observador Luenberger clásico. Este desarrollo tiene como principal característica que la matriz de ganancia es obtenida empleando el procedimiento de desigualdades matriciales lineales (LMI).

La otra aplicación emplea un observador híbrido, que se compone de un observador Luenberger, desarrollado bajo el esquema mencionado anteriormente, y un observador asintótico. La selección del observador más adecuado se realiza por medio de un índice de funcionamiento que monitoriza la validez de los observadores utilizados.

Además se desarrolla una extensión del observador híbrido para aplicarse a un esquema multifrecuencial, utilizando las medidas disponibles en línea y las realizadas fuera de línea y con retardo.

Estas propuestas se comparan con un observador asintótico clásico y un filtro Kalman extendido, mostrándose los resultados de la misma bajo condiciones de simulación. 


\section{Resum}

El desenvolupament d'observadors per a l'estimació de variables i paràmetres en un procés biotecnològic és el tema central del present treball. S'aborda així el desenvolupament d'un observador d'estats que considera en el seu disseny les incerteses presents en el modelatge del sistema, definides estes dins d'un politop on els vèrtexs són els límits d'operació del sistema.

Aquest desenvolupament es presenta en dos vessants. Una és l'aplicació del concepte a un observador Luenberger clàssic, que té com a principal característica que la matriu de guany és determinat emprant el procediment de desigualtats matricials lineals (LMI).

L'altra aplicació és un observador híbrid, que es compon d'un observador Luenberger, desenvolupat davall l'esquema mencionat anteriorment, i un observador asimptòtic, que per mitjà d'un paràmetre que depèn del model fa que l'observador trie entre un o altre tipus.

A més, es desenvolupa una extensió de l'observador híbrid per a aplicarse a un esquema multifreqüencial, el quin es definix per mitjà de les mesures disponibles en línia i les mesures realitzades fora de línia i amb retard.

Estes propostes es comparen amb un observador asimptòtic clàssic i un filtre de Kalman estès, mostrant-se els resultats de la mateixa baix condicions de simulació. 


\section{Abstract}

The development of the estimation observers of variables or parameters in a biotechnological process is the main topic in the present work. It talks about the development of a state observer that considers in its design the uncertainty of the model in the system, which are defined inside of the bidimensional polygon or tridimensional polyhedral sides that limit the operation of this system.

This development shows itself in two facets. The first one, is the application of a concept to a Luenberger classic observer, which has as its main characteristic that the gain matrix is determined by following the procedure of linear matrix inequality (LMI).

The second facet is about a hybrid observer that is made up by a Luenberger observer developed like the previous one I have mentioned, and also by a curved observer that helps the observer transit freely among other observers by a parameter. Besides, it will develop an extension of the hybrid observer to be applied to a multifrequency scheme, defined by available linear measures and measures out of the line and delayed.

These theories can be compared to a classic asymptotic observer and an extended Kalman filter, showing results under the same simulation conditions. 


\section{Agradecimientos}

A las instituciones:

De España, la Universidad Politécnica de Valencia (UPV), de México, la Dirección General de Educación Superior Tecnológica (DGEST), el Programa SUPERA de la Asociación Nacional de Universidades e Instituciones de Educación Superior (ANUIES) y el Instituto Tecnológico de Mérida (ITM) por su apoyo económico y administrativo para el desarrollo de este proyecto.

A mis maestros:

Especialmente al Dr. José Luis Navarro Herrero por su apoyo y amistad, al Dr. Jesús Pico Marco por sus acertadas colaboraciones para el trabajo, al Dr. Pedro Albertos y el Dr. Julián Salt por sus consejos y atenciones.

A quienes me apoyaron moralmente:

A mi madre, Consuelo, mis hermanos, Ruby, Luis y Julia, mis cuñados, sobrinos y sobrinas, a la familia Chiu, la familia Nazaralá, la familia Cervera Flores, Don Juan y Doña Alicia, por su grandísimo apoyo moral y por todo aquello que nunca podré pagarles y les estaré eternamente agradecidos.

A quienes son la principal razón de mi vida:

Alis, mi amada esposa, Karen Faride y Raúl Esteban, mis queridos hijos, a quienes les robe vacaciones, fines de semana, descansos, por seguir con este sueño. Los amo.P.

Y gracias a Dios, a Jesús y al Espirítu Santo, que son el soporte de mi vida, de mi ser y de mi corazón. 
Las oportunidades se dan una vez en la vida, tómala o déjala ir, y sigue siempre adelante

Luis Chiu Ruiz $(\dagger)$

En memoria, gracias por la vida. 



\section{Índice general}

Pag.

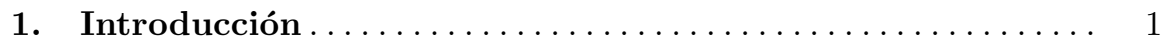

1.1. Principios básicos de operación de un biorreactor ......... 1

1.2. Motivación ............................ 2

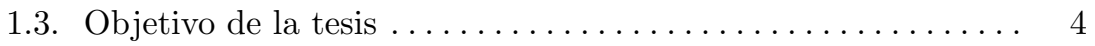

1.3.1. Objetivo principal ...................... 4

1.3.2. Objetivos particulares..................... 4

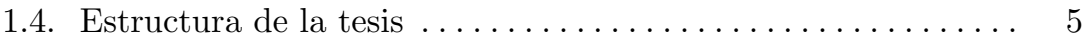

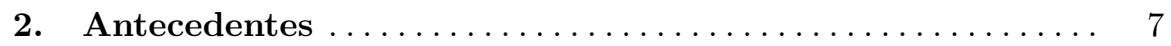

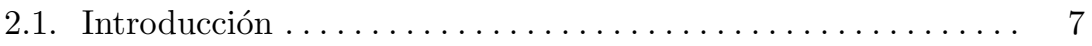

2.2. Modelado de biorreactores .................... 8

2.2.1. Modelo dinámico general . . . . . . . . . . . . . 10

2.3. Observabilidad ............................ 11

2.4. Modelo general de un observador $\ldots \ldots \ldots \ldots \ldots \ldots \ldots \ldots . \ldots \ldots$

2.4.1. Observador de Luenberger ................. 15

2.4.2. Filtro Kalman ....................... 17

2.4.3. Observadores no lineales ..................... 19

2.5. Observador Asintótico ...................... 20

2.5.1. Observador asintótico para bioprocesos . . . . . . . . . 20

2.5.2. Observador asintótico de bucle cerrado .......... 22

2.5.3. Observador híbrido ................... 23

2.6. Otros esquemas de observadores ................. 26

2.6.1. Desigualdades Matriciales Lineales (LMI)-Principios

básicos ............................... 26

2.6.2. Observador empleando LMI _............... 29

2.6.3. Observador de intervalo .................... 31

2.6.4. Estimadores de Horizonte Móvil ............... 32

2.6.5. Observadores multifrecuenciales .............. 33

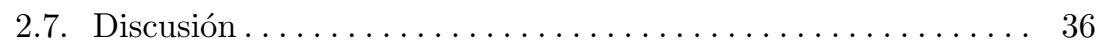

3. Observador para bioprocesos empleando LMI ......... 37

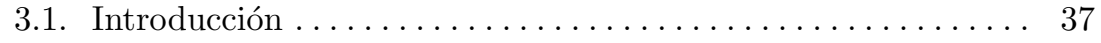

3.2. Análisis del modelo ......................... 38 
XII Índice general

3.3. Teorema del valor medio diferencial . . . . . . . . . . . . . . 40

3.3.1. Aplicación de DMVT al modelo dinámico general . . . 42

3.4. Análisis de convergencia . . . . . . . . . . . . . . . . 43

3.5. Análisis del Algoritmo del Observador . . . . . . . . . . . . . . . 44

3.6. Discusión ........................... 47

4. Observador multifrecuencial empleando LMI . . . . . . . . 49

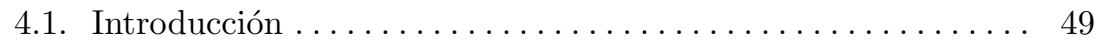

4.2. Antecedentes . . . . . . . . . . . . . . . . . . . 49

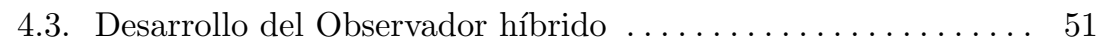

4.3.1. Resolución mediante DMVT .............. 52

4.4. Observador híbrido multifrecuencial . . . . . . . . . . . . 53

4.4.1. Observador híbrido durante las medidas periódicas

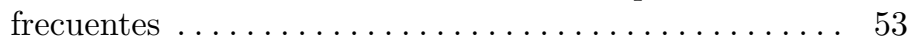

4.4.2. Observador híbrido durante las medidas esporádicas . . 54

4.4.3. Análisis de estabilidad ................. 55

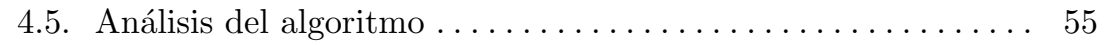

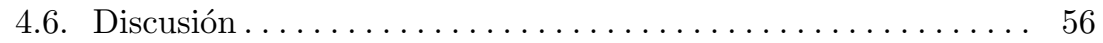

5. Casos de Estudio $\ldots \ldots \ldots \ldots \ldots \ldots \ldots \ldots \ldots \ldots \ldots$

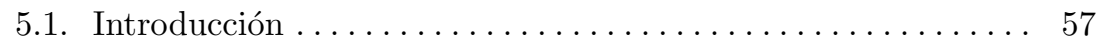

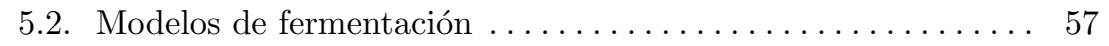

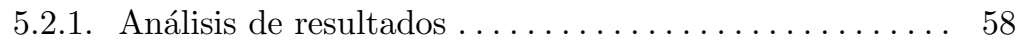

5.3. Modelo de fermentación - Sistema Productor de Biomasa . . . 60

5.3.1. Análisis de Observabilidad . . . . . . . . . . . . . 61

5.3.2. Observador Luenberger empleando LMI . . . . . . . . . 61

5.3.3. Observador Kalman Extendido............... 62

5.3.4. Observador Asintótico ................. 62

5.3.5. Resultados ...................... 63

5.4. Modelo de fermentación - Sistema productor metabólico . . . . 64

5.4.1. Descripción del modelo................... 65

5.4.2. Análisis de observabilidad . . . . . . . . . . . . . 66

5.4.3. Análisis de las Jacobianas . . . . . . . . . . . . . 68

5.4.4. Observador Luenberger empleando LMI . . . . . . . . . 69

5.4.5. Observador asintótico ................. 70

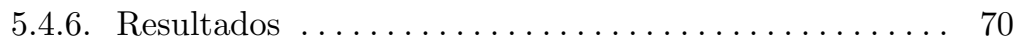

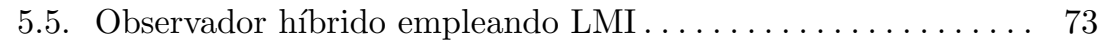

5.6. Modelo de fermentación - Sistema multifrecuencial . . . . . . . . 75

5.6.1. Descripción del observador híbrido multifrecuencial . . 77

5.6.2. Resultados ..................... 79

5.7. Discusión ......................... 81

6. Conclusiones y Trabajos futuros $\ldots \ldots \ldots \ldots \ldots \ldots \ldots \ldots$

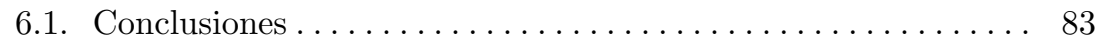

6.2. Trabajos futuros $\ldots \ldots \ldots \ldots \ldots \ldots \ldots \ldots \ldots \ldots \ldots \ldots$ 
Índice general XIII

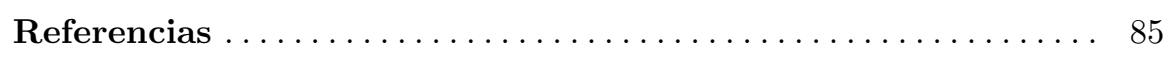





\section{Índice de figuras}

Figura Pag.

5.1. Evolución del error de estimación de la biomasa $\ldots \ldots \ldots \ldots 63$

5.2. Evolución del error de estimación de la biomasa cuando se simula que $\mu_{m}=0,3 h^{-1} \ldots \ldots \ldots \ldots \ldots \ldots \ldots \ldots \ldots \ldots \ldots$

5.3. Simulación del bioproceso . .................. 66

5.4. Comportamiento del error relativo en la estimación del sustrato. $e_{O L}$ : error empleando un observador Luenberger, $e_{O A}$ : error empleando un Observador Asintótico, $e_{O K}$ : error empleando un Filtro Kalman ................... 71

5.5. Evolución del error relativo de observación ante la presencia de ruido en la medida ....................... 71

5.6. Evolución del error relativo de observación cuando existe variación en el parámetro $\mu_{\max } \ldots \ldots \ldots \ldots \ldots \ldots \ldots \ldots \ldots \ldots$

5.7. Evolución del error relativo de observación cuando la tasa de disolución $\mathrm{D}$ es un escalón de 0.1 a $0.2 \mathrm{l} / \mathrm{h} \ldots \ldots \ldots \ldots \ldots . \ldots 73$

5.8. Evolución del error relativo de observación. Error del observador Luenberger extendido empleando LMI $(O L)$. Error del observador híbrido empleando LMI $(O L A)$. Error del observador asintótico $(O A) \quad \ldots \ldots \ldots \ldots \ldots \ldots \ldots \ldots \ldots \ldots \ldots$

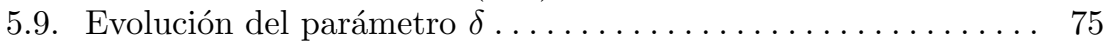

5.10. Evolución del error absoluto ante la presencia de variaciones en $\mu_{\max }$. Error del observador Luenberger extendido empleando LMI $(O L)$. Error del observador híbrido empleando LMI $(O L A)$. Error del observador asintótico $(O A) \quad 76$

5.11. Información disponibles del modelo .............. 77

5.12. Estimación de Sustrato y Etanol empleando Observador Asintótico (línea azul) y un Observador Híbrido (línea negra) . 80

5.13. Valores de la medida y de la estimación en instantes esporádicos , (.) Medida real (+) Observador Asintótico, (*) Observador Híbrido ............................ 80 



\section{Introducción}

Se que Dios no me dará algo que yo no pueda manejar, solo deseo que El no confié demasiado en mí

Madre Teresa

La industria biotecnológica se ha expandido rápidamente debido a los avances en la comprensión de los sistemas biológicos complejos y a la alta demanda de sus productos en las áreas de alimentación, farmacéutica, especialidades químicas, etc.

Un proceso bioquímico (o bioproceso) típico consiste en una etapa de reacción, en la cuál un gran número de células son empleadas para sintetizar el producto deseado, seguido por una serie de etapas de separación, en la cuál el producto es recuperado. Un requerimiento clave para el proceso de bioproducción es la identificación de un microorganismo capaz de producir el producto bioquímico deseado.

El control de procesos juega un importe papel en ésta industria. La escasez de los sensores en línea que permita el monitoreo en tiempo real de los estados del proceso representa uno de los principales obstáculos en el control de bioprocesos.

El desarrollo de sensores por software ha permitido sustituir parcialmente la necesidad de los sensores en línea. Elemento importante de estos es el diseño de observadores, siendo sobre esta área se enfoca el trabajo de la tesis. En la misma se propone un observador, el cuál mejora la convergencia respecto a los desarrollos de observadores clásicos, con la capacidad de manejar la incertidumbre existente en la medida y en el modelado del bioproceso.

\subsection{Principios básicos de operación de un biorreactor}

Un reactor bioquímico (o biorreactor) es un recipiente en el que se desarrolla una población de microorganismos vivos, cuya densidad deberá ser lo suficientemente grande $\left(\sim 10^{13}\right.$ celulas/l) para garantizar su crecimiento, [Henson, 2006]. La masa de estos organismos (bacterias, levaduras, hongos, etc) se denomina biomasa.

Las células son alimentadas continuamente con ciertos nutrientes, que contienen carbono, nitrógeno, sales, minerales, etc. que deberán de representar 
el ambiente de crecimiento adecuado del microorganismo para favorecer su desarrollo o la producción de sustancias de interés para el sistema. Estos nutrientes se denominan substrato.

El flujo de salida del biorreactor contendrá componentes que no fueron consumidos durante el proceso, biomasa celular que se desarrolló, así como el producto que las células excretaron si se dieron las condiciones ambientales de operación adecuadas. En caso contrario el producto se quedará dentro de la célula siendo el escenario poco favorable para la bioproducción, aun cuando en proceso posterior sea posible recuperarlo.

El producto deseado consiste en el desarrollo de las células en sí mismas o un producto del metabolismo (por ejemplo el etanol), que deberá de separarse del resto de los componentes a través de diversos procesos de recuperación y/o purificación posteriores.

Otro flujo de salida también presente son los gases emitidos por el metabolismo, como por ejemplo el dióxido de carbono.

La manera en que se suministre el substrato $\left(F_{\text {ent }}\right)$ y de extracción del producto $\left(F_{\text {sal }}\right)$ determina el tipo de proceso de producción, siendo estos:

- Por lotes $\left(F_{\text {ent }}=F_{\text {sal }}=0\right)$

- Semi-lote, es decir por lotes con alimentación de substrato $\left(F_{\text {ent }}>0, F_{\text {sal }}=\right.$ $0)$

- tipo continuo $\left(F_{\text {ent }}=F_{\text {sal }}>0\right)$

La operación efectiva del biorreactor requiere además que las condiciones ambientales de desarrollo del metabolismo sean las adecuadas, incluyendo condiciones de esterilidad, agitación adecuada de la mezcla, condiciones de temperatura y $\mathrm{pH}$ controlados, control del flujo de nutrientes, etc.

Todas estas condiciones representan el ámbito de medición y control de un bioproceso, siendo fundamental la medida de las variables de estado que caracterizan el comportamiento del proceso: las concentraciones de biomasa, substrato y producto.

Las medidas disponibles de las variables ambientales pueden dar en forma indirecta información adicional de las condiciones en que evoluciona el proceso, dado que se cuenta con sensores para determinar en línea el valor de la temperatura, $\mathrm{pH}$, el oxígeno disuelto, $\mathrm{CO}_{2}$ en los gases de salida, velocidad de agitación, entre otros.

\subsection{Motivación}

La instrumentación y sensores disponibles para bioprocesos no siempre cubren todas las medidas deseables o aún las necesarias. En estos procesos, las variables internas importantes que presentan dificultades de medición, pero que caracterizan al estado y progreso de la fermentación, son las concentraciones de biomasa, substrato y producto secundario. 
A pesar de los recientes desarrollos en sensores de ion selectivo y enzimáticos, métodos ópticos y por medida de la impedancia [Johnson, 1987],[Kammona et $\sim a l ., 1999]$ muchas de las variables de concentración en una fermentación de fase líquida no pueden medirse en línea con exactitud y confiabilidad. Por consiguiente, los análisis y ensayos de laboratorio son requeridos para soportar las acciones de control y supervisión de las fermentaciones.

Cuando combinamos un sensor que permite la medida en línea de alguna de las variables de importancia en el bioproceso, junto con la medida de la señal de entrada y un modelo del proceso, entonces se puede estimar en línea, bajo ciertas condiciones y mediante un algoritmo, las variables de estado y/o parámetros cuyo acceso en línea no es sencillo.

Este es el mecanismo subyacente al concepto denominado sensor por software ("softsensor").

Si el sensor por software es empleado para la estimación de variables de estado, entonces se denomina observador de estado o simplemente observador, y si se emplea para la estimación de los parámetros internos del modelo, entonces se denomina estimador de parámetros [Bastin and Dochain, 1990].

Muchos trabajos emplean un mecanismo de observación basado en la disponibilidad de un modelo, como por ejemplo el filtro Kalman, el observador Luenberger y los observadores de alta ganancia. Todos ellos son observadores exponenciales que permiten el uso de parámetros de sintonización para manejar la tasa de convergencia de la estimación del estado al estado real.

$\mathrm{Su}$ principal inconveniente es que sus resultados requieren la disponibilidad de un modelo bioquímico de la fermentación lo suficientemente exacto. Además requieren que el proceso sea completamente observable a partir de las salidas secundarias.

Debido a las características no lineales de las dinámicas de los bioprocesos es de interés extender estos conceptos y explotar estructuras particulares para aplicaciones a problemas de ingeniería bioquímica.

Las versiones linealizadas (el modelo de linealización tangencial) de las dinámicas de los procesos se calcula a partir de una expansión en serie de Taylor de un modelo de espacio de estado alrededor de algún punto de equilibrio. La teoría del observador lineal entonces puede ser aplicada sobre estos modelos linealizados.

El Filtro Kalman es uno de los algoritmos mas populares, ya que es fácil de implementar, dado que el algoritmo puede obtenerse directamente del modelo de espacio de estado. Sin embargo, dado que está basado en un modelo linealizado del proceso (al igual que el observador de Luenberger extendido), las propiedades de estabilidad y convergencia son esencialmente locales y validas alrededor del punto de equilibrio.

Esta condición hace que se dificulte garantizar la estabilidad sobre un amplio rango de operación. Así, si el filtro Kalman no se inicializa adecuadamente, la estimación puede desviarse o, más aún, divergir.

Por otra parte, G. Bastin y D. Dochain [1990], desarrollaron un observador 
asintótico que es considerado un método que se diferencia de los observadores clásicos (Luenberger y filtro Kalman) los cuales requieren un conocimiento amplio del modelo del proceso, y del observador adaptativo, el cuál incluye un estimador de estados y parámetros dentro del mismo esquema.

El observador asintótico está basado en el modelo dinámico no lineal del proceso y, junto con un numero de medidas en línea que dependen del número de reacciones presentes, se emplea para estimar los estados no medidos y los parámetros desconocidos del proceso.

$\mathrm{Su}$ principal desventaja recae en que la velocidad de convergencia depende de los condiciones de operación establecidas por la tasa de disolución.

Ahora bien, existen otros esquemas de desarrollo de algoritmos de estimación que consideran las incertidumbres en el modelo del proceso, incertidumbre en la medida, rangos permitidos de variación del error en la estimación, intervalos de variación de los parámetros, etc. Todos y cada uno de ellos buscan mejorar la velocidad de convergencia y optimizar los tiempos de cómputo.

La motivación principal de la tesis es desarrollar un observador de estados cuyo algoritmo este basado en un modelo dinámico no lineal acotado por un politopo, cuyos intervalos se definen en base a las cotas de los estados y parámetros conocidos.

\subsection{Objetivo de la tesis}

\subsubsection{Objetivo principal}

Desarrollar un observador de estados para procesos biotecnológicos que permita considerar dentro de su diseño la incertidumbre en el modelo del proceso y los intervalos de operación de los parámetros y estados que lo definen, permitiendo con ello contar con un observador cuya velocidad de convergencia se mejore en forma sustancial y soporte una mayor gama de variaciones en los parámetros.

\subsubsection{Objetivos particulares}

1. Analizar del estado del arte de los algoritmos observadores de estados y parámetros aplicados a los bioprocesos considerando sus principales ventajas y desventajas.

2. Desarrollar un observador de estados que incluya las incertidumbres en el modelo, acotado mediante un politopo que se define en base a los intervalos de operación, y aplicarlo a bioprocesos.

3. Establecer y diseñar de un observador multifrecuencial, el cuál considere las medidas fuera de línea para corregir la convergencia del observador monofrecuencia, empleando para ello un algoritmo basado en un modelo acotado por un politopo. 


\subsection{Estructura de la tesis}

La presente tesis se encuentra estructurada de la siguiente manera: En el capítulo 2 se realiza el análisis de algoritmos de estimación empleados para la estimación de estados y parámetros. Los observadores clásicos (filtro Kalman, observador Luenberger, observador de alta ganancia), el observador asintótico típico y algunas de sus variantes (observador asintótico de bucle cerrado y observadores híbridos) y por último otros observadores que tienen aplicación en bioprocesos (observadores que emplean la resolución mediante LMI, observadores de intervalo) son analizados.

El capítulo 3 aborda el desarrollo del observador para bioprocesos basado en un observador Luenberger extendido en el cuál el modelo del bioproceso está acotado por un politopo.La determinación del mismo se basa en el Teorema del Valor Medio Diferencial, y el uso de LMI. Esto permite considerar incertidumbre en el modelo, caracterizada como intervalos que acotan los estados del bioproceso.

En el capítulo 4 se extiende el concepto anterior aplicándolo a un observador híbrido. Este se forma por un observador Luenberger extendido y un asintótico, considerando ahora el comportamiento multivariable del sistema. Es decir, ciertas medidas se disponen mediante sensores en línea y otras se disponen fuera de línea analizadas en el laboratorio. Estas últimas son las que se estiman mediante el sensor por software y cuando se dispongan de los resultados del análisis fuera de línea, permitirá la corrección en la estimación y así garantizar la convergencia y estabilidad del sensor por software.

La aplicación de estos casos se muestra en el capítulo 5 mostrando sus resultados y comparándolos con los resultados que se obtienen en la implementación de otros algoritmos clásicos, exponiendo las ventajas y desventajas del desarrollo.

Por último, el capítulo 6 describe las conclusiones del trabajo así como los trabajos futuros que se pueden desarrollar a partir de la presente propuesta. 



\section{Antecedentes}

Las ciencias aplicadas no existen, solo las aplicaciones de la ciencia

Louis Pasteur

\subsection{Introducción}

La medición de la concentración de los principales componentes en los bioprocesos es de gran utilidad, ya que permiten la monitorización y control en línea del proceso. Pero en muchos casos, estas mediciones involucran diversos problemas. Por ejemplo:

- Los sensores son muy caros.

- Estos deben de esterilizarse.

- Pueden degradar las propiedades hidrodinámicas de biorreactores complejos.

- Las técnicas de medición son frecuentemente destructivas

- A menudo están disponibles solamente muestras discretas, no necesariamente periódicas, y algunas veces con un tiempo largo de análisis fuera de línea.

Debido a que diversas variables se encuentran involucradas y no todas pueden determinarse en línea, el uso de sensores por software es una de las herramientas más empleadas en la actualidad.

Un sensor por software, [Chéruy, 1997], [Bogaerts, 1999], emplea mediciones en línea (en forma continua) empleando sensores físicos y un algoritmo de estimación. El algoritmo es llamado un observador de estado cuyo acierto es proporcionar una estimación de estado incluyendo el valor real del estado del proceso.

Este algoritmo requiere conocer el modelo matemático del proceso, así como un modelo de medición que relacione la variable medida con las variables del proceso.

El presente capítulo presenta una revisión del modelo dinámico general, siendo el modelo basado en balance de masas que más se emplea para el modelado de bioprocesos. Este modelo es del tipo no estructurado-no segregado, es decir un modelo simplificado que no refleja todos los detalles, de manera tal que se asumen errores en el modelado y en los parámetros. 
También se presenta una revisión del esquema general de un observador y de los algoritmos de estimación que comúnmente se emplean para la estimación de estados. Estos son los observadores exponenciales, observadores asintóticos y sus variantes, así como otros esquemas de observadores.

\subsection{Modelado de biorreactores}

El desarrollo de diversos productos por medio de fermentación requiere que el proceso sea monitoreado y controlado de forma adecuada, logrando con ello un producto de calidad. El fermentador o reactor biológico es un tanque en el cuál varias reacciones químicas ocurren simultáneamente en un medio líquido. Estas reacciones pueden ser de dos tipos, [Bastin and Impe, 1997]:

Reacciones de crecimiento microbiano: Estas consisten en el crecimiento de microorganismos o generación de producto $(\mathrm{P})$ proveniente del consumo de nutrientes apropiados o sustrato (S). La masa de microorganismos vivos en el reactor es llamado la biomasa (X).

Reacciones de catálisis enzimática: El sustrato (S) es transformado en un producto $(\mathrm{P})$ por medio de la acción catalítica de una enzima $(\mathrm{E})$, la cuál es una proteína producida por microorganismos vivos:

- enzimas extracelulares secretadas al medio líquido: $S+E \rightarrow P+E$

- enzimas intracelulares que se quedan en la célula, de tal forma que la biomasa trabaja como catalizador: $S+X \rightarrow P+X$

Estas reacciones que ocurren dentro del fermentador se definen mediante modelos matemáticos, los cuales son de singular importancia para el diseño, optimización, monitorización y control de sistemas químicos y bioquímicos.

El modelado matemático de biorreactores es un problema cambiante debido a la complejidad del metabolismo celular. El grado de complejidad está determinado por factores tales como la cantidad de conocimiento de su comportamiento, los datos requeridos para construcción del modelo y validación, requerimientos computacionales de procesamiento y uso del modelo.

Una clasificación de los modelos de biorreactores se plantea a nivel de descripción de la fase biótica, [Romero, 2003]:

\section{Nivel fisiológico}

- Modelo estructurado. Expresan la fisiología del proceso de crecimiento biológico, generalmente de forma descriptiva debido a su complejidad .

- Modelo no estructurado.Describen las características de crecimiento respecto a la concentración de una o varias sustancias y su transformación a otras.

Nivel población 
- Modelo segregado. Se caracteriza la población por algún rasgo que lo diferencie, considerando la estructura poblacional de los microorganismos.

- Modelo no segregado. Se asume que el estado fisiológico es el mismo para todos los microorganismos.

Los modelos más empleados en aplicaciones de control corresponden a los modelos no estructurado-no segregados debido a su sencillez. El modelado de sistemas de reacción de estos modelos está basado en conceptos de balance de masas y energía, así como en leyes de termodinámica de la forma ${ }^{1}$ [King et $\sim$ al., 2002] :

$$
\frac{d x_{j}}{d t}=\sum_{i=1}^{n r} \nu_{i j} r_{i}+\frac{F_{i}}{V}, j=1, \ldots, n c
$$

Esta expresión describe la evolución temporal de las concentraciones $\left(x_{j}\right)$ de los nc componentes en una reacción, las cuales cambian debido a las alimentaciones $\left(F_{i}\right)$ y a las $n r$ reacciones $\left(r_{i}\right)$ que ocurren dentro de las sustancias, ponderadas por los coeficientes pseudo-estequiométricos $\left(\nu_{i j}\right)$.

Las tasas de reacción tienen una alta dependencias entre concentraciones, diferentes trabajos se han dedicado a su definición y un esquema general de estas se muestra el cuadro 2.1.

Una de las expresiones más usadas para las tasas de reacción es la número

Cuadro 2.1. Algunas dependencias entre concentraciones de expresiones de tasas de reacción. Las cinéticas 6 a 16 son típicas en bioprocesos

\begin{tabular}{|l|l|l|l|}
1 & $x_{1}^{a 1}$ & 9 & $\frac{a_{2} x_{1} x_{3}}{\left(a_{1}+x_{1}\right)\left(a_{2}+x_{2}\right)}$ \\
2 & $x_{1} x_{2}$ & 10 & $\frac{a_{2} x_{3} x_{4}}{\left(a_{1}+x_{1}\right)\left(a_{2}+x_{2}\right)\left(a_{4}+x_{4}\right)}$ \\
3 & $x_{1}^{a 1} x_{2}^{a 2}$ & 11 & $\frac{x_{1} x_{2} x_{3}}{\left(a_{1}+x_{1}\right)\left(a_{3}+x_{3}\right)}$ \\
4 & $x_{1}^{a 1} x_{2}^{a 2} x_{3}^{a 3}$ & 12 & $\frac{x_{1} x_{2} x_{3}}{a_{1}+x_{1}}$ \\
5 & $\frac{x_{1}^{a 1} x_{2}^{a 2}}{1+a_{3} x_{1}^{a}}$ & 13 & $\frac{a_{2} x_{1} x_{3} x_{4}}{\left(a_{1}+x_{1}\right)\left(a_{2}+x_{2}\right)}$ \\
6 & $\frac{x_{1} x_{2}}{a_{1}+x_{1}}$ & 14 & $\frac{x_{1} x_{2}}{a_{1}+x_{1}+a_{2} x_{2}^{a 3}}$ \\
7 & $\frac{x_{1} x_{2}}{a_{1}+x_{1}+a_{2} x_{2}}$ & 15 & $\frac{x_{1} x_{2}}{a_{1} x_{1}+x_{2}}$ \\
8 & $\frac{x_{x_{1} x_{2}}}{a_{1}+x_{1}+\left(x_{1} / a_{2}\right)^{2}}$ & 16 & $a_{1} x_{2}+\frac{x_{1} x_{2}}{a_{2}+x_{1}}$ \\
\hline
\end{tabular}

6 denominado cinética de Monod así como la expresión número 8 que se denomina de Haldane.

Para lograr el potencial biológico completo, las condiciones ambientales deberán mantenerse en valores óptimos. Involucrados en el proceso, es requerida

\footnotetext{
${ }^{1}$ No son las ecuaciones desde un punto de vista pseudo-estequiométrico estricto, solo aparecen las ecuaciones de las concentraciones de los compuestos de interés en el estudio
} 
la medición en línea de diversas variables como $\mathrm{pH}$, Oxígeno disuelto, Oxígeno liberado $\left(O_{2}\right)$, flujo de aire, flujo de sustancias para controlar el $\mathrm{pH}$, velocidad de agitación, temperatura, $\mathrm{CO}_{2}$, densidad óptica (DO), así como el control del nivel de espuma para evitar la contaminación del medio.

Además, también es deseable determinar el estado actual de la concentración de la biomasa, sustrato y producto. Algunos métodos de medición empleados por los sensores más comunes para estos últimos se muestra en la tabla 2.2. Cuando no se cuenta con el sensor específico para la medida deseada, es

Cuadro 2.2. Algunos métodos de medición empleados por los sensores para fermentadores

\begin{tabular}{ll}
\hline Variable & Método \\
\hline Biomasa & Densidad óptica \\
Sustrato & Electrodo enzimático, microbial \\
Etanol & Tubing de Teflon \\
Oxígeno Disuelto & Celdas galvánicas \\
Dióxido de Carbono Infrarojo
\end{tabular}

común emplear variables relacionadas para estimarlas [Stephanopoulos and San, 1984]. Por ejemplo, considerando un modelo de fermentación de levadura, medir la fracción parcial del oxígeno y del dióxido de carbono junto con la medida del flujo de entrada de aire permiten determinar la tasa de consumo de oxígeno (OUR, oxygen uptake rate) y la tasa de producción de dióxido de carbono (CER, carbon dioxide evolution rate).

Ambos parámetros, junto con la tasa de alimentación de glucosa (GFR, glucose feedrate), permiten estimar la tasa de producción de biomasa (BPR, biomass production rate), [Johnson, 1987]:

$$
B P R=2,17 * O U R-3,26 * C E R+6,52 * G F R
$$

\subsubsection{Modelo dinámico general}

La dinámica del proceso biotecnológico (o bioproceso) se obtiene del balance de masas y energía y puede representarse mediante el modelo de espacio de estado no lineal introducido en [Bastin and Dochain, 1990]:

$$
\dot{\xi}=K \varphi(\xi(t))-D(t) \xi(t)+F(t)-Q(t)
$$

donde

- $\xi(t) \in \mathbb{R}^{n}$ son los componentes o variables de estado,

- $K \varphi(\xi(t))$ describe la cinética de las reacciones bioquímicas y microbiológicas, siendo $K \in \mathbb{R}^{n x m}$ la matriz de los coeficientes de producción y $\varphi \in \mathbb{R}^{m}$ el vector de las tasas de reacción 
- $D \in \mathbb{R}^{n}$ es la tasa de dilución, definida por la velocidad del flujo de entrada

$\left(F_{i}\right)$ dividida por el volumen del sistema $(\mathrm{V})$

- $F(t) \in \mathbb{R}^{n}$ es el vector de la tasa de alimentación externa

- $Q(t)$ representa los flujos de salida en forma gaseosa

Los bioprocesos tienen el inconveniente de que los sensores físicos para la medida en línea de todos los estados no siempre se tienen disponibles. Algunas medidas están disponibles pero estas deben de ser analizadas en el laboratorio antes de obtener el valor de la variable deseada.

Schügerl [2001] presenta una revisión de los desarrollos en ingeniería de bioprocesos, incluyendo la monitorización del proceso de formación del producto por análisis de inyección de flujo, cromatografía, espectroscopía así como los biosensores, entre otros.

Así, en el contexto de la observación de los estados de un bioproceso podemos dividir estos en un grupo de estados que se miden en línea y en forma frecuente $\xi_{1} \in \mathbb{R}^{q}(q<n)$ y los estados restantes de los cuales tenemos su medida fuera de línea y en forma esporádica $\xi_{2} \in \mathbb{R}^{n-q}$ :

$$
\begin{aligned}
\xi & =\left[\begin{array}{ll}
\xi_{1} & \xi_{2}
\end{array}\right]^{T} \\
\xi_{1} & =L \xi
\end{aligned}
$$

donde $L \in \mathbb{R}^{n}$ es la matriz que selecciona los componentes medidos de $\xi$. A efectos de este trabajo, se consideran las siguientes condiciones respecto a los sistemas a analizar:

Condición 1 (C1):

- $u(t)=F(t)-Q(t)$ son las entradas/salidas del proceso.

- $y(t)=L \xi(t)=\xi_{1}(t)$ : se cuenta con sensores que permiten determinar la evolución de uno o más estados en línea.

- $m \leq n$ el número de reacciones es menor o igual al número de componentes.

- $\operatorname{rank}(K)=r_{k}$ la matriz $K \in \mathbb{R}^{m}$ es de rango completo.

\subsection{Observabilidad}

Por definición, un sistema se dice que es observable si y solo si es posible determinar cualquier estado (con condición inicial arbitraria) $x(t)=x_{i}$ usando solamente un registro finito de la salida, $y(\tau)$ para $t \leq \tau \leq T$, [Friedland, 1987]. Considerando un sistema no lineal del tipo:

$$
\begin{aligned}
& \dot{x}=f(x, u) \\
& y=h(x, u)
\end{aligned}
$$

donde $x(t) \in \mathbb{R}_{w}^{n}$, es el estado del sistema y $y(t) \in \mathbb{R}^{m}, u(t) \in \mathbb{R}^{l}$, son la salida medida y la entrada exógena, cuyas funciones $f$ y $h$ son funciones suaves de tiempo $t$. Consideremos que $\varrho(t, x, u)$ es la trayectoria de la solución del estado correspondiente a la entrada $u$ y estado inicial $x$ evaluada en pequeños 
intervalos $t$.

Si tenemos dos puntos $x_{1}$ y $x_{2}$ dentro del universo del sistema, $x_{1}, x_{2} \in \mathbb{R}^{n}$, y tomamos una entrada $u$ entonces podemos tener las siguientes definiciones, [Wang and Sontag]:

- $x_{1}$ y $x_{2}$ son indistinguibles para $u, x_{1} \sim x_{2}$ si

$$
h\left(\varrho\left(t, x_{1}, u\right), u(t)\right)=h\left(\varrho\left(t, x_{2}, u\right), u(t)\right)
$$

para cualquier entrada $u$ y cualquier tiempo $t \geq 0$. En caso contrario, se dice que $x_{1}$ y $x_{2}$ son distinguibles para $u, x_{1} \nsim x_{2}$.

- Si para cualesquiera dos estados, $x_{1} \sim x_{2}$ implica que $x_{1}=x_{2}$ entonces el sistema es observable.

El objetivo es determinar las condiciones del sistema (2.5) que permitan la reconstrucción de $x$, a través de la observación de $y$, [Vecchio and Murray, 2003]. Consideremos los siguientes conceptos:

- $\mathcal{U}=\left(u_{0}, u_{1}, u_{2}, \ldots\right)$ la secuencia infinita de control $u=u_{0}$ y sus derivadas

$$
\begin{gathered}
\dot{u}=u_{1} \\
\dot{u}_{1}=u_{2}
\end{gathered}
$$

:

$$
\dot{u}_{k}=u_{k+1}
$$

y $\mathcal{X}=\left(x, u_{0}, u_{1}, \ldots\right)=(x, \mathcal{U})$

- La derivada de Lie de una función $\varrho: \mathbb{R}^{\infty} \rightarrow \mathbb{R}$ es:

$$
\varrho_{i+1}=L_{f} \varrho_{i}(\mathcal{X})=\sum_{i=1}^{n} \frac{\partial \varrho(\mathcal{X})}{\partial x_{i}} f_{i}+\sum_{k=0}^{\infty} \frac{\partial \varrho(\mathcal{X})}{\partial u_{k}} u_{k+1}
$$

- Definimos $\psi(\mathcal{X})=\left(\varrho_{0}, \varrho_{1}, \ldots, \varrho_{n}\right)$

Definición Un sistema definido por 2.5 es observable localmente si y solo si

$$
\operatorname{rank}(\mathcal{O} \mathcal{L})=\operatorname{rank}\left(\frac{\partial \psi(\mathcal{X})}{\partial x}\right)=n
$$

Si $\varrho(\mathcal{X})=h\left(x, u_{0}\right)$ entonces $\varrho_{0}=h \mathrm{y}$ :

$$
\varrho_{i+1}=L_{f} h(\mathcal{X})=\sum_{i=1}^{n} \frac{\partial h\left(x, u_{0}\right)}{\partial x_{i}} f_{i}\left(x, u_{0}\right)+\frac{\partial h\left(x, u_{0}\right)}{\partial u_{0}} u_{1}
$$

Para sistemas no lineales, es adecuado también definir el concepto de observabilidad uniforme:

Definición: Un sistema se dice es observable uniformemente cuando es observable para cualquier entrada limitada $u(t)$ y en cualquier intervalo de tiempo $[0, T], T>0$, tal que el estado inicial está determinado basandose en la salida $y(t)$ y la entrada $u(t)$, [Gauthier and Bornard, 1981], [Gauthier et ${ }^{\sim}$ al., 1992]. 


\subsection{Modelo general de un observador}

Una condición para el monitoreo y operación efectiva de un proceso es tener acceso continuo a las variables del proceso. Las variables esenciales en un proceso pueden dividirse en dos grandes grupos:

- Las variables de estado (aquellas que únicamente determinan el estado del proceso)

- Los parámetros del proceso (aquellos que afectan el estado del proceso no las variables de estado)

El uso de observadores de estados y/o parámetros cuando no se dispone de información en forma directa o en línea es de gran importancia para los sistemas de control. A continuación se describe el modelo general de un observador para posteriormente analizar algunos esquemas clásicos de observadores.

El modelo de espacio de estado de un sistema no lineal está definido por:

$$
\begin{aligned}
& \dot{x}=f(x, u) \\
& y=h(x)
\end{aligned}
$$

siendo $x$ las variables de estado del sistema, $u$ las entradas al sistema, $y$ las variables medidas. El esquema general de un observador de estado para este sistema se define como, [Dochain, 2003]:

$$
\dot{\hat{x}}=f(\hat{x}, u)+K(\hat{x})(y-\hat{y})
$$

donde $\hat{x}$ y $\hat{y}$ son la estimación en línea de $x$ y $y$, mediante:

$$
\hat{y}=h(\hat{x})
$$

y $K(\hat{x})$ es la ganancia del observador.

Si definimos el error de observación como $e=x-\hat{x}$, la dinámica del error será:

$$
\dot{e}=f(\hat{x}+e, u)-f(\hat{x}, u)-K(\hat{x})(h(\hat{x}+e)-h(\hat{x}))
$$

El diseño del observador de estado consiste en elegir una ganancia apropiada $K(\hat{x})$ tal que la dinámica del error anterior tenga las propiedades deseadas. Para hacerlo se puede emplear consideraciones deterministicas (observador de Luenberger) o estocásticas (filtro de Kalman).

El observador Luenberger es adecuado para sistemas estables, aunque sus resultados no siempre son óptimos. Por otro lado, el filtro Kalman minimiza el error entre las cantidades medidas y las cantidades estimadas tomando en cuenta las incertidumbres en la medida y en el modelo.

Dado que los bioprocesos son un sistema no lineal y estas dos opciones son para sistemas lineales, la alternativa es emplear el concepto de "extendido" que se aplica al observador Luenberger y al filtro Kalman. La idea de los 
observadores extendidos es obtener un modelo linealizado a lo largo de la trayectoria del estado y entonces diseñar un observador de estados local ${ }^{2}$. Si consideramos la linealización de la ecuación (2.11) alrededor del error de observación $e=0$ obtendremos:

$$
\frac{d e}{d t}=[A(\hat{x})-K(\hat{x}) C(\hat{x})] e
$$

donde:

$$
A(\hat{x})=\left[\frac{\partial f}{\partial x}\right]_{x=\hat{x}} \quad C(\hat{x})=\left[\frac{\partial h}{\partial x}\right]_{x=\hat{x}}
$$

Así, una clase general de observador de estado para el sistema no lineal de la ecuación (2.2) será:

$$
\begin{aligned}
\dot{\hat{\xi}} & =K \varphi(\hat{\xi})-D \hat{\xi}+u(t)+\gamma[y-\hat{y}] \\
y & =L \xi
\end{aligned}
$$

donde $\hat{\xi}$ es la estimación de $\xi, \gamma \in \mathbb{R}^{q x n}$ es la matriz de ganancia que depende de $\hat{\xi}$ y $L \in \mathbb{R}^{n x 1}$ es la matriz que selecciona los componentes medidos de $\xi$. Esta ecuación es una copia del modelo con un término de corrección proporcional al error de observación de los estados medidos.

El error de observación se define entonces como:

$$
e=\xi-\hat{\xi}
$$

la dinámica del error depende la siguiente ecuación diferencial:

$$
\frac{d e}{d t}=K[\varphi(\hat{\xi}+e)-\varphi(\hat{\xi})]-D e-\gamma L e
$$

El error de observación $e=0$ es un punto de equilibrio de la ecuación (2.16). Linealizando alrededor de éste se puede analizar el comportamiento local del observador, en especial sus propiedades de convergencia local:

$$
\frac{d e}{d t}=[A(\hat{\xi})-\gamma L] e
$$

donde:

$$
A(\hat{\xi}) \doteq K\left[\frac{\partial \varphi(\xi)}{\partial \xi}\right]_{\xi=\hat{\xi}}-D I_{n}
$$

Asumase que el sistema de la ecuación (2.2) es exponencialmente observable mediante la estructura de (2.14) cuyo principal problema de diseño es

${ }^{2}$ Esto es válido para sistemas parsimoniosos, es decir, que no presentan fuertes variaciones de comportamiento 
la selección de la ganancia $\gamma$. Para ello las soluciones clásicas son el observador de Luenberger y filtro Kalman extendido, así como el observador de alta ganancia. A continuación se realiza una revisión de estos tres esquemas de observadores ampliamente usados en la estimación de estados y parámetros de un bioproceso.

\subsubsection{Observador de Luenberger}

En el observador de Luenberger el objetivo es determinar el valor de $\gamma$ tal que el error tienda asintóticamente a cero, para lo cuál se deberán de cumplir con ciertas restricciones:

1. La matriz $A(\hat{\xi})-\gamma L$ y sus derivadas están definidas

$$
\begin{aligned}
|A(\hat{\xi})-\gamma L| & \leq N_{1} & & \forall \hat{\xi} \\
\left|\frac{d}{d t}\{A(\hat{\xi})-\gamma L\}\right| & \leq N_{2} & & \forall \hat{\xi}
\end{aligned}
$$

2. Los valores propios de la matriz $A(\hat{\xi})-\gamma L$ tienen parte real negativa:

$$
\mathbb{R} e\left\{\lambda_{i}[A(\hat{\xi})-\gamma L]\right\} \leq N_{3}<0 \quad \forall \hat{\xi} \quad i=1 . . n
$$

La rapidez con que el error $e=\xi-\hat{\xi}$ converga asintóticamente a cero depende de los valores propios de la matriz $A(\hat{\xi})-\gamma L$ los cuales deberán ser reales negativos. El procedimiento general a seguir para determinar los valores de la matriz de ganancias es:

- Definimos el polinomio característico del observador $\lambda[A(\hat{\xi})-\gamma L]=\lambda^{n}+a_{n-1} \lambda^{n-1}+\cdots+a_{1} \lambda+a_{0}=0$

- Se define el polinomio característico deseado $\left(\lambda-\lambda_{n}\right)\left(\lambda-\lambda_{n-1}\right) \ldots\left(\lambda-\lambda_{1}\right)=0$

- Igualar los coeficientes para determinar $\gamma$

Para este procedimiento, podemos emplear la fórmula de Ackermann, o bien, en Arnold and Datta [1998] se plantean varios esquemas para la asignación de polos que pueden utilizarse.

Problemas de la linealización La linealización del modelo dinámico general mediante la ecuación (2.18) está fundamentada en la Jacobiana de las tasas de reacción. En los procesos biotecnológicos, si son de tipo continuo, el equilibrio se alcanza cuando $S>0$ para un flujo de entrada $F_{i}$ determinado. En semicontinuo, para que $S$ sea constante se requiere que el flujo de alimentación crezca exponencialmente, en consecuencia lo hará el volumen, así que existirá un punto de equilibrio en $S \geq 0$.

Dado que normalmente el flujo se encuentra acotado, $S \rightarrow 0 \mid F_{i}=F_{\max }$, si 
el sustrato ha disminuido a un valor estable y este valor es cercano a 0 , entonces algunos de las Jacobianas tenderán a cero cuando el sistema alcance el equilibrio

Consideremos las expresiones del cuadro 2.1, especificamente las cinéticas 6 (Monod) y 8 (Haldane). Si $\frac{\partial r n}{\partial x}$ es el Jacobiano de la expresión $r$ con respecto a la(s) concentración(es).

$$
\begin{aligned}
& \frac{\partial r 6}{\partial x}=\left[\begin{array}{c}
\frac{x_{2}}{a_{1}+x_{1}}-\frac{x_{1} x_{2}}{\left(a_{1}+x_{1}\right)^{2}} \\
\frac{x_{1}}{a_{1}+x_{1}}
\end{array}\right] \\
& \frac{\partial r 8}{\partial x}=\left[\begin{array}{c}
x_{2}\left(a_{1}+x_{1}+\frac{x_{1}{ }^{2}}{a_{2}^{2}}\right)^{-1}-x_{1} x_{2}\left(1+2 \frac{x_{1}}{a_{2}^{2}}\right)\left(a_{1}+x_{1}+\frac{x_{1}{ }^{2}}{a_{2}{ }^{2}}\right)^{-2} \\
x_{1}\left(a_{1}+x_{1}+\frac{x_{1}{ }^{2}}{a_{2}{ }^{2}}\right)^{-1}
\end{array}\right]
\end{aligned}
$$

Si consideramos que:

$$
\begin{array}{ll}
x_{1} \doteq S & \text { Sustrato } \\
x_{2} \doteq X & \text { Biomasa } \\
x_{3} \doteq P & \text { Producto }
\end{array}
$$

entonces el límite de las Jacobianas cuando $x_{1}=S$ tiende a cero es:

$$
\begin{aligned}
& \lim _{x_{1} \rightarrow 0} \frac{\partial r 6}{\partial x}=\left[\begin{array}{c}
\frac{x_{2}}{a_{1}} \\
0
\end{array}\right] \\
& \lim _{x_{1} \rightarrow 0} \frac{\partial r 8}{\partial x}=\left[\begin{array}{c}
\frac{x_{2}}{a_{1}} \\
0
\end{array}\right]
\end{aligned}
$$

Dado que para determinar las ganancias del observador Luenberger, se emplean las Jacobianas de las tasas de reacción y como algunas tienden a cero, esta condición puede producir un valor no deseado de dichas ganancias.

Ejemplo 2.2 Como un ejemplo de este planteamiento, consideremos el caso planteado en Bastin and Dochain [1990]:

$$
\begin{aligned}
\frac{d S}{d t} & =-k_{1} \varphi(\xi)-D S+D S_{i n} \\
\frac{d X}{d t} & =\varphi(\xi)-D X
\end{aligned}
$$

donde la velocidad de reacción se define por la ley de Contois:

$$
\varphi(\xi)=\frac{\mu_{\max } X S}{K_{c} X+S}
$$

Las ganancias para el Luenberger propuesto son: 


$$
\begin{aligned}
& \gamma_{1}=-\lambda_{1}-\lambda_{2}-k_{1} \hat{\varphi}_{S}+\hat{\varphi}_{X}-2 D \\
& \gamma_{2}=\frac{1}{k_{1} \hat{\varphi}_{X}}\left(-\lambda_{1} \lambda_{2}-\left(\lambda_{1}+\lambda_{2}\right)\left(D-\hat{\varphi}_{X}\right)-\left(D-\hat{\varphi}_{X}\right)^{2}+k_{1} \hat{\varphi}_{X} \hat{\varphi}_{S}\right)
\end{aligned}
$$

donde

$$
\begin{array}{r}
{\left[\begin{array}{c}
\hat{\varphi}_{X} \\
\hat{\varphi}_{S}
\end{array}\right]=\left[\begin{array}{l}
\frac{\partial \varphi}{\partial X} \\
\frac{\partial \varphi}{\partial S}
\end{array}\right]=\left[\begin{array}{c}
\frac{\mu_{\max } \hat{S}^{2}}{(K c \hat{X}+\hat{S})^{2}} \\
\frac{\mu_{\max } K c \hat{X}^{2}}{(K c \hat{X}+\hat{S})^{2}}
\end{array}\right]} \\
\lim _{S \rightarrow 0}\left[\begin{array}{c}
\hat{\varphi}_{X} \\
\hat{\varphi}_{S}
\end{array}\right]=\left[\begin{array}{c}
0 \\
\frac{\mu_{\max } K c \hat{X}^{2}}{K c \hat{X}^{2}}
\end{array}\right]
\end{array}
$$

Entonces como consecuencia $\omega_{2}$ tenderá a valores de ganancia muy grandes debido a $\hat{\varphi}_{X}$, afectando la convergencia del observador. De esta forma, es deseable que cuando se determine la matriz de ganancia del observador, esta considere los intervalos de operación de los estados para evitar la divergencia en la estimación.

\subsubsection{Filtro Kalman}

El Filtro Kalman es un conjunto de ecuaciones que proporcionan una solución recursiva del método de mínimos cuadrados publicado originalmente en 1960 por R.E. Kalman. El filtro es adecuado principalmente para sistemas lineales.

El filtro Kalman intenta estimar un estado de un proceso definido este por una ecuación estocástica diferencial lineal. Sin embargo, la gran mayoria de los procesos son no lineales. Cuando se realiza una linealización alrededor del valor medio actual y se aplica un filtro Kalman, entonces esto se conoce como Filtro Kalman Extendido (extended Kalman filter, EKF).

Se puede definir entonces al EKF como un estimador de estados que aproxima óptimamente las reglas de Bayes por medio de linealización, [Welch and Bishop, 2002].

El principal objetivo es calcular $\gamma$ tal que minimice el error cuadrático de observación:

$$
E=\int_{0}^{t}\|e(\tau)\|^{2} d \tau
$$

La ganancia $\gamma$ entonces es igual a:

$$
\gamma=R(\hat{\xi}) L^{T}
$$

donde la matriz $R \in \mathbb{R}^{n x n}$ es generada por la ecuación Riccati:

$$
\frac{d R}{d t}=-R L^{T} L R+R A(\hat{\xi})^{T}+A(\hat{\xi}) R
$$

Existe diversa literatura que trata sobre el desarrollo del algoritmo del filtro Kalman, las cuales principalmente se centran en tres tipos de esquemas 
dependiendo de la naturaleza del modelo y de la medición, [Crassidis and Junkins, 2004]:

- Discreto: donde ambos modelo y medición tienen dinámica de tiempo discretos.

- Continuo: ambos modelo y medición tienen dinámica de tiempo continuo.

- Continuo-Discreto: involucra que el modelo es continuo en el tiempo y la medición es discreta tomada de un procesador digital de señales.

Aplicación en bioprocesos Dada la popularidad que tuvo el filtro Kalman, su uso en bioprocesos ha sido muy difundido y realizado con algunas modificaciones, buscando siempre la mejora de la convergencia debido a los problemas que presenta la linealización de la función alrededor del valor local.

Stephanopoulos and San [1984] presentan un completo estudio de la estimación en línea de biorreactores, empleando para ello un filtro Kalman extendido. Presentan resultados satisfactorios de estimación de estados y parámetros, bajo condiciones de estado estable y considerando la medición proporcionada por el análisis de gases fuera de línea de una fermentación.

Por su parte, Kozub and MacGregor [1992] plantea una alternativa al usar un segundo filtro para mejorar la estimación de los estados iniciales. El esquema es empleado en un reactor de polimerización tipo semi continuo, obteniendo un filtro robusto para un amplio rango de perturbaciones desconocidas y errores en el modelo.

Rusell et $\sim$ al. [2000], presenta una alternativa similar empleando un esquema de filtro Kalman extendido en cascada( Cascaded EKF-CEKF). En este se emplea el segundo filtro para la estimación de las condiciones iniciales y lo compara con el uso de un suavizador de punto fijo(Fixed-Point SmootherFPS).

Este esquema es aplicado al monitoreo inferencial de la calidad basado en modelo de sistemas en lote y semicontinuo. Los mejores resultados se dan con el FPS cuando se requiere la estimación en un punto fijo en el pasado. Un problema del CEKF es que presenta una alta demanda computacional.

En resumen, el filtro Kalman extendido es una excelente alternativa de filtrado cuando existe la presencia de ruido, sin embargo:

- Existen problemas de convergencia y de estabilidad

- La posibilidad de una desviación en la estimación esta presente debido a la dependencia de la exactitud en el modelo del proceso.

- La sintonización del estimador representa un trabajo en ocasiones tedioso.

- El tiempo de cómputo requerido para resolver las ecuaciones del observador puede ser largo debido a que se integran los estados y la matriz de ganancia en cada iteración. 


\subsubsection{Observadores no lineales}

Un observador no lineal tiene como objetivo principal la estimación asintótica del estado-parámetro desconocido diseñándose a partir del modelo no lineal del proceso sin realizar ninguna linealización. Esto es importante ya que los errores se puedan dar en el modelado del proceso, limitando el rendimiento de un observador.

Farza et ${ }^{\sim}$ al. [1997], realiza una comparación de convergencia de la realización continua y discreta del observador desarrollado para un sistema no lineal, analizando el efecto del parámetro de sintonización en la estrategia del observador de alta ganancia.

Existen diversos desarrollos de observadores no lineales, siendo el más clásico el observador de alta ganancia, el cuál se describe a continuación.

Observador de alta ganancia La construcción de un algoritmo de estimación necesita cumplir con la propiedad de observabilidad. Esto es, dada una entrada $u$, la habilidad para reconstruir el estado $x(t)$ basándose en el histórico de los datos de salida $y$ en el intervalo de tiempo $[0, T]$.

Para sistemas lineales, la propiedad de observabilidad no depende de las entradas $u(t)$. Por el contrario, para sistemas no lineales, la propiedad de observabilidad si depende de las entradas. El concepto de observabilidad uniforme definida anteriormente se relaciona con la existencia de un observador de alta ganancia, [Gauthier et ${ }^{\sim}$ al., 1992].

Dado un sistema del tipo:

$$
\begin{gathered}
\dot{x}=F(x)+G(x) u \\
y=C x
\end{gathered}
$$

con $x \in \mathbb{R}^{n}, u \in \mathbb{R}^{m}, y \in \mathbb{R}, F(x)=\left[x_{2}, x_{3}, \ldots, x_{n}, \varphi(x)\right]^{T}, G(x)=$ $\left[g_{1}\left(x_{1}\right), g_{2}\left(x_{1}, x_{2}\right), \ldots, g_{n}\left(x_{1}, \ldots, x_{n}\right)\right], C=(1,0, \ldots, 0)$ con las siguientes condiciones:

- la entrada $u(t)$ está acotada uniformente: $|u(t)| \leq u_{0}, u_{0}>0$

- $\mathrm{G}(\mathrm{x})$ es Lipschitz globalmente

- $\frac{\partial G_{i}}{\partial x_{j}}=0, i=1, \ldots, n-1, j=i+1, \ldots, m$

El sistema:

$$
\dot{\hat{x}}=F(\hat{x})+G(\hat{x}) u-S_{\infty}^{-1} C^{\prime}(C \hat{x}-y)
$$

donde $S_{\infty}$ es la solución de:

$$
0=-\theta S_{\infty}-A^{\prime} S_{\infty}-S_{\infty} A+C^{\prime} C
$$

para $\theta$ suficientemente grandes y con $A: \mathbb{R}^{n} \rightarrow \mathbb{R}^{n}, A_{i, j}=\delta_{i, j-1}$ es un observador de alta ganancia con: 


$$
\|\hat{x}(t)-x(t)\|=\leq K \exp (-\theta t / 3)\left\|\hat{x}_{0}-x_{0}\right\|
$$

Como se observa, el observador es una copia del sistema con un término de corrección que inyecta la salida.

Los observadores de alta ganancia tienen ventajas y desventaja similares al observador Luenberger y al filtro Kalman. Si consideramos la estabilidad teórica, el error de observación puede ser pequeño si se seleccionan valores altos de ganancia, pero a su vez puede generar grandes oscilaciones o variaciones en presencia de ruido.

Si los errores de observación son asintóticamente pequeños, estos errores estarán relacionados con los estados del sistema transformado, no con los estados físicos originales.

Su aplicación a bioprocesos se ha reportado para la estimación de estados y parámetros usando técnicas algebraicas, [Martínez-Guerra etªl., 2001b]. También para el diagnóstico en bioprocesos, es decir la detección, estimación y fallas en el proceso, [Martínez-Guerra et $\left.{ }^{\sim} a l ., 2001 \mathrm{a}\right]$. Se ha reportado combinado con un observador Luenberger para realizar la cancelación de las no linealidades del modelo y simplificar el diseño del observador, [Tornambe, 1989].

\subsection{Observador Asintótico}

El observador asintótico es considerado un método intermedio entre observadores exponenciales, los cuales requieren un conocimiento amplio del modelo del proceso, y el observador adaptativo, el cuál incluye un estimador de estados y parámetros dentro del mismo esquema, cuya principal aplicación está orientada a los procesos biotecnológicos.

En esta sección se presenta el desarrollo clásico del observador asintótico desarrollado y analizado por Bastin y Dochain, analizando después algunas de sus variantes como son el observador de lazo cerrado y combinado con algún observador exponencial, los cuales se denominan observadores híbridos.

\subsubsection{Observador asintótico para bioprocesos}

El observador asintótico, [Bastin and Dochain, 1990] parte del modelo dinámico general, ecuación (2.2), considerando que la matriz $\mathrm{K}$ y $D \xi_{i}$ son conocidos y no se conoce la estructura de las tasas de reacción $\varphi(\xi)$. El diseño del observador se basa en la partición del estado $\xi=\left[\xi_{a}, \xi_{b}\right]^{T}$ con lo cuál tendremos también la división de $K=\left[K_{a}, K_{b}\right]^{T}, u=\left[u_{a}, u_{b}\right]^{T}$ y se debe cumplir con:

Condición :

$K_{a}$ es una submatriz de $K$ de rango completo $\operatorname{rank}\left(K_{a}\right)=r_{k}$. 
Entonces, existe una transformación definida por:

$$
Z=A_{0} \xi_{a}+\xi_{b}
$$

donde $A_{0}$ es la solución de la ecuación matricial $A_{0} K_{a}+K_{b}=0$. Así, las ecuaciones que definen al modelo dinámico general son equivalentes a:

$$
\begin{aligned}
\dot{\xi_{a}} & =K_{1} \varphi\left(\xi_{a}, \xi_{b}\right)-D \xi_{a}+u_{a} \\
\dot{Z} & =-D Z+A_{0} u_{a}+u_{b}
\end{aligned}
$$

Donde $Z$ es independiente de las tasas de reacción $\varphi(\xi)$.

Si reescribimos ahora $Z$ como una combinación lineal de los vectores de los estados medidos en línea $\left(\xi_{1}\right)$ y de los estados medidos fuera de línea $\left(\xi_{2}\right)$ :

$$
Z=A_{0} \xi_{a}+\xi_{b}=A_{1} \xi_{1}+A_{2} \xi_{2}
$$

Así el observador asintótico se obtiene de la ecuación (2.35) sustituyendo a $Z$ por su estimación $\hat{Z}$ y junto con la ecuación que define la estimación de los estados medidos fuera de línea, ecuación (2.37):

$$
\left\{\begin{array}{l}
\dot{\hat{Z}}=-D \hat{Z}+A_{1} u_{1}+A_{2} u_{2} \\
\hat{\xi}_{2}=A_{2}^{-1}\left(\hat{Z}-A_{1} \xi_{1}\right)
\end{array}\right.
$$

Podemos considerar algunos casos de acuerdo a las siguientes condiciones:

\section{Condición:}

- El número de estados medidos ( $\left.\operatorname{dim} \xi_{1}\right)$ debe ser igual o mayor al rango de la matriz $\mathrm{K}$

- $A_{2}$ es invertible

Caso 1: $p=m$. Es decir, los estados medidos son iguales al número de reacciones, con lo cuál $A_{2}$ tiene una inversa definida:

$$
\left\{\begin{array}{l}
\dot{\hat{Z}}=-D \hat{Z}+A_{1} u_{1}+A_{2} u_{2} \\
\hat{\xi}_{2}=A_{2}^{-1}\left(\hat{Z}-A_{1} \xi_{1}\right)
\end{array}\right.
$$

Caso 2: $p>m$. En este caso, el número de componentes medidos es mayor al número de reacciones:

$$
\left\{\begin{array}{l}
\dot{\hat{Z}}=-D \hat{Z}+A_{1} u_{1}+A_{2} u_{2} \\
\hat{\xi}_{2}=A_{2}^{+}\left(\hat{Z}-A_{1} \xi_{1}\right)
\end{array}\right.
$$

donde $A_{2}^{+}$es la seudoinversa de la matriz $A_{2}$

La dinámica del error de estimación es igual a:

$$
\begin{aligned}
& e=\xi_{2}-\hat{\xi}_{2}=A_{2}^{-1} Z-A_{2}^{-1} \hat{Z} \\
& \dot{e}=\dot{Z}-\dot{\hat{Z}}=-D e
\end{aligned}
$$


El error de estimación de este observador depende de la tasa de disolución D y requiere que $\mathrm{D}$ no sea cero por un periodo prolongado de tiempo (esto se cumple con los reactores semicontinuos y continuos).

La principal ventaja que presenta el observador asintótico es la independencia de las tasas de reacción, las cuales muchas veces es desconocida. Por el contrario, su naturaleza de bucle abierto hace que presente ciertas desventajas, [Chachuat and Bernard, 2002], en particular se asume implícitamente que la parte de balance de masas del modelo se conoce perfectamente y no presenta desviaciones ni se encuentra corrompida.

En conclusión, el observador asintótico presenta las siguientes características:

- Es más simple que el filtro Kalman

- Su estabilidad es buena bajo ciertas condiciones del proceso, siendo totalmente dependiente de la tasa de dilución.

- El costo computacional es bajo, comparado con el filtro Kalman extendido.

- No se requiere conocer el modelo de la tasa de reacción.

\subsubsection{Observador asintótico de bucle cerrado}

Para resolver los problemas que presenta el observador asintótico debido a su naturaleza de bucle abierto, en Romero [2003] se plantea una alternativa de corrección empleando para ello un elemento de retroalimentación como el que se mostró en el modelo general de los observadores:

$$
\hat{\xi_{2}}=A_{2}^{+}\left(\hat{Z}-A_{1} \xi_{1}\right)+\gamma\left(\xi_{1}-\hat{\xi_{1}}\right)
$$

Este esquema de corrección considera el conocimiento de la estructura de las cinéticas de reacción. Dado que la estimación de los estados medidos se define por medio de la integración numérica del modelo:

$$
\dot{\xi_{1}}=K_{1} \varphi\left(\xi_{1}, \hat{\xi_{2}}\right)-D \xi_{1}+u_{1}
$$

y la matriz $\gamma$ es definida en base a la ecuación de la dinámica del error de estimación:

$$
\begin{aligned}
& e=\xi_{2}-\hat{\xi_{2}} \\
& \dot{e}=-\left(D I+\gamma K_{1} \Psi\left(\xi_{1}, \xi_{2}^{*}\right)\right) e=-\Upsilon e
\end{aligned}
$$

donde $\Psi$ se define:

$$
\Psi\left(\xi_{1}, \xi_{2}^{*}\right)=\left.\frac{d \Phi\left(\xi_{1}, \hat{\xi}_{2}\right)}{d \xi_{2}}\right|_{\left(\xi_{1}, \xi_{2}^{*}\right)}
$$

siendo $\xi_{2}^{*}$ el estado estimado comprendido entre $\xi_{2}$ y $\hat{\xi}_{2},-D e$ la dinámica del error del observador asintótico de lazo abierto. Entonces, la matriz $\gamma$ se define como 


$$
\gamma=\Upsilon\left[K_{1} \Psi\left(\xi_{1}, \xi_{2}^{*}\right)\right]^{-1}
$$

El valor de $\Upsilon$ deberá de garantizar la convergencia del observador.

La matriz $\gamma$ también puede definirse empleando los esquemas descritos anteriormente, es decir, empleando ubicación de polos por Ackermann como en el Observador Luenberger Extendido:

$$
\gamma=P(A(\xi))(\mathcal{O})^{-1}\left[\begin{array}{c}
0 \\
\vdots \\
1
\end{array}\right]
$$

donde $\mathcal{O}$ es la matriz de observabilidad o usando la ecuación de Ricatti como en el Observador Kalman Extendido, tal que:

$$
\gamma=R L^{T}
$$

En Bernard et ${ }^{\sim}$ al. [2000] se plantea también una solución para un observador asintótico de bucle abierto, sin embargo esta requiere de otras medidas que permitan dar mayor información sobre la evolución de los estados. Por otro lado, en la propuesta de Romero [2003] el término de corrección depende del error en la medida y, además, este término permite fijar la velocidad de convergencia del observador.

\subsubsection{Observador híbrido}

Un observador de estados híbrido es la combinación de un observador asintótico con: (a) un observador Luenberger extendido [Hulhoven et ${ }^{\sim}$ al., 2006], (b) un observador Kalman extendido [Bogaerts, 1999], (c) un observador de alta ganancia [Gouzé and Lemesle, 2001], [Lemesle and Gouzé, 2005].

Partiendo de la separación de los estados en medidos en línea y medidos fuera de línea y aplicando el concepto del modelo general para observadores, el principio básico es introducir un parámetro que permita moverse de un observador exponencial a un observador asintótico. Este parámetro dependerá de la calidad del modelo dinámico.

Luenberger-Asintótico De la separación de los estados definidos en el observador asintótico y con la variable de estado auxiliar $Z(t)=A_{1} \xi_{1}+A_{2} \xi_{2}$ se redefine el modelo dinámico general como:

$$
\left\{\begin{array}{l}
\dot{\xi}_{1}=K_{1} \varphi(\xi)-D \xi_{1}+u_{1} \\
\dot{Z}=-D Z+A_{1} u_{1}+A_{2} u_{2} \\
\xi_{2}=A_{2}^{+}\left(Z-A_{1} \xi_{1}\right)
\end{array}\right.
$$

El observador extendido de Luenberger para este modelo es , [Hulhoven et ${ }^{\sim}$ al., 2006]: 


$$
\left\{\begin{array}{l}
\dot{\hat{\xi}}_{1}=K_{1} \varphi(\hat{\xi})-D \hat{\xi}_{1}+u_{1}+\gamma_{1}(\hat{\xi})\left(y-\hat{\xi}_{1}\right) \\
\dot{\hat{Z}}=-D \hat{Z}+A_{1} u_{1}+A_{2} u_{2}+\gamma_{z}(\hat{\xi})\left(y-\hat{\xi}_{1}\right) \\
\hat{\xi}_{2}=A_{2}^{+}\left(\hat{Z}-A_{1} \hat{\xi}_{1}\right)
\end{array}\right.
$$

La dinámica del error de estimación para este observador híbrido se define como:

$$
\begin{aligned}
& \dot{\epsilon}_{1}=K_{1}\{\varphi(\xi)-\varphi(\hat{\xi})\}-D \epsilon_{1}-\gamma_{1} \epsilon_{1} \\
& \dot{\epsilon}_{z}=-D \epsilon_{z}-\gamma_{z} \epsilon_{1}
\end{aligned}
$$

donde $\epsilon_{1}=\xi_{1}-\hat{\xi}_{1}$ y $\epsilon_{z}=Z-\hat{Z}$.

Para tomar en cuenta la calidad del modelo dinámico, se emplea la siguiente definición:

$$
\xi_{1} \rightarrow \delta \xi_{1}+(1-\delta) y
$$

Así, el parámetro $\delta$ tiene como valores al intervalo $[0,1]$ y esto hace que el observador resultante sea una combinación de un observador Luenberger extendido y un asintótico, de acuerdo al error en la estimación de $\xi_{1}$. Si $\delta \equiv 1$, corresponderá a un observador Luenberger extendido. Si $\delta \equiv 0$ corresponderá a un observador asintótico

Kalman-Asintótico Considerando una transformación de los estados definido por:

$$
\begin{aligned}
& Z_{1}=\xi_{1} \\
& Z_{2}=\xi_{2}+(1-\delta) A_{0} \xi_{1}
\end{aligned}
$$

donde el parámetro $\delta=[0,1]$, si $\delta=0$ la transformación es equivalente a la propuesta por Bastin and Dochain [1990], y para $\delta=1$ podemos hacer un cambio de notación : $Z_{2}=\xi_{2}$. El observador híbrido asintótico con un filtro Kalman continuo discreto extendido está dado por, [Bogaerts, 1999]:

- Predicción (continuo)

$$
\begin{aligned}
\frac{d \hat{Z}_{1}}{d t} & =K_{1} \varphi\left(\hat{Z}_{1}, \hat{\xi_{2}}\right)-D \hat{Z}_{1}+u_{1} \\
\frac{d \hat{Z}_{2}}{d t} & =\delta K_{2} \varphi\left(\hat{Z}_{1}, \hat{\xi_{2}}\right)-D \hat{Z}_{2}+u_{2}+(1-\delta) A_{0} u_{1} \\
\hat{\xi_{1}} & =\hat{Z}_{1} \\
\hat{\xi_{2}} & =\hat{Z}_{2}-(1-\delta) A_{0} \xi_{1} \\
\frac{d P}{d t} & =F(\hat{Z}) P+P F^{T}(\hat{Z}) \quad \forall t \in\left[t_{k}, t_{k+1}[\right.
\end{aligned}
$$

- Corrección (discreto)

$$
\begin{aligned}
\left.K\left(t_{k}\right)\right) & =P\left(t_{k}^{-}\right) C^{T}\left[C P\left(t_{k}^{-}\right) C^{T}+Q\left(t_{k}\right)^{-1}\right] \\
\hat{Z}\left(t_{k}^{+}\right) & =\hat{Z}\left(t_{k}^{-}\right)+K\left(t_{k}\right)\left(y\left(t_{k}\right)-\hat{\xi}_{1}\left(t_{k}^{-}\right)\right) \\
P\left(t_{k}^{+}\right) & =P\left(t_{k}\right)-K\left(t_{k}\right) C P\left(t_{k}^{-}\right)
\end{aligned}
$$


Este algoritmo permite recuperar a un filtro Kalman continuo-discreto extendido cuando $\delta=1$ y al observador asintótico cuando $\delta=0$.

Observador de error limitado Un observador de error limitado es un sistema dinámico tal que no requiere que el error entre las variables estimadas y modeladas convergan a cero pero si que esté limitado a un valor constante que sea menor al error en la medición.

Así, para un sistema dinámico

$$
\left\{\begin{array}{l}
\dot{x}(t)=f(x(t), u(t)) \\
y(t)=h(x(t))
\end{array}\right.
$$

se define un observador

$$
\hat{\dot{x}}(t)=f_{1}(\hat{x}(t), u(t), y(t))
$$

cuyo error está limitado por una constante real positiva

$$
\lim _{t \rightarrow \infty}\|\hat{x}(t)-x(t)\| \leq m \quad m \in \mathbb{R}>0
$$

Esta constante dependerá, en el caso de los bioprocesos [Gouzé and Lemesle, 2001], por ejemplo del conocimiento que se tenga de las tasas de reacción.

Si consideramos que $\mu_{p}(\xi)$ es el conocimiento parcial de la tasa de reacción y $\mu_{t}(\xi)$ es el conocimiento perfecto de la misma (por ejemplo una función Monod), entonces el conocimiento de la tasa de reacción $\mu(\xi)$ se define en base a:

$$
\left|\mu_{t}(\xi)-\mu_{p}(\xi)\right| \leq a \quad a \in \mathbb{R}>0
$$

en este caso, $m$ depende de $a$. Por ejemplo,[Lemesle and Gouzé, 2005], consideremos el siguiente sistema:

$$
\left\{\begin{array}{l}
\dot{X}=\mu(S) X-D X \\
\dot{S}=-k_{s} \mu(S) X+D S_{i}-D S
\end{array}\right.
$$

si realizamos el cambio de variable $(X, S) \rightarrow(Z, S)$, tal que el estado medido es $\mathrm{S}$, donde:

$$
Z=k_{s} X+\theta S
$$

siendo $\theta>1$ un valor constante, la dinámica de $\mathrm{Z}$ está dada por:

$$
\hat{Z}=(1-\theta) k_{s} X \mu(S)-D Z+\theta D S_{i}
$$

Entonces, el observador de error limitado para este sistema es:

$$
\dot{\hat{Z}}=(1-\theta) k_{s} \hat{X} \hat{\mu}(S)-D \hat{Z}+\theta D S_{i}
$$

donde $\hat{\mu}(S)$ se elige de forma tal que se cumpla con la siguiente condición:

$$
\left|\mu_{t}(S)-\mu_{p}(S)\right| \leq a \quad a \in \mathbb{R}>0
$$

El error estático depende del valor de $\theta$ : 
- si $\theta>1$ entonces el observador se comporta como un observador de alta ganancia $\dot{\hat{Z}}=(1-\theta) k_{s} \hat{X} \hat{\mu}(S)-D \hat{Z}+\theta D S_{i}$

- si $\theta=1$ entonces se comporta como un observador asintótico $\dot{\hat{Z}}=-D \hat{Z}+$ $D S_{i}$

\subsection{Otros esquemas de observadores}

El análisis de un modelo matemático puede contener ciertos elementos de incertidumbre, las cuales pueden deberse a ruido interno o externo. También pueden influir las no linealidades como histéresis o fricción, pobre conocimiento de la planta, incertidumbres, o parámetros que varían lentamente.

En esta sección se presentan algunos esquemas de observadores no lineales que consideran las incertidumbres en la medida o en la planta en el diseño del observador. Se presentan entonces casos tales como el observador empleando LMI y el observador de intervalo, así como el concepto de observador multifrecuencial.

\subsubsection{Desigualdades Matriciales Lineales (LMI)-Principios básicos}

En los años recientes, las desigualdades matriciales lineales (Lineal Matrix Inequality-LMI) ha emergido como una herramienta para los problemas del control que parecen difíciles de solucionar de una manera analítica. Aunque la historia de LMI's se remonta a la década de los cuarenta con un énfasis importante de su papel en control en los años 60, sólo las recientes técnicas numéricas que se han desarrollado para LMI's permiten tener una solución de una manera práctica y eficiente.

Varios paquetes de software de Matlab están disponibles que permiten una codificación simple de los problemas generales de LMI y de los que se presentan en problemas típicos del control (LMI Control Toolbox, LMI-tool, YALMIP).

Alentado por la disponibilidad de paquetes de software que resuelven LMI's, la investigación en control robusto ha experimentado un cambio de paradigma. Esto es, en lugar de llegar a una solución analítica, la intención es reformular un problema dado a verificar si un LMI tiene solución, o funciona óptimamente sobre restricciones de LMI.

Una definición básica de LMI establece que es una desigualdad tal que $F(x)>0$ donde $F$ es una función afín mapeada a un espacio vectorial dimensional finito $\mathbb{X}$ para matrices Hermetianas o Simétricas, [Scherer and Weiland, 2004]. Su forma canónica se define como: 


$$
\begin{array}{r}
F(x)=F_{0}+\sum_{i=1}^{m} x_{i} F_{i}>0 \\
F(x)=F_{0}+x_{1} F_{1}+\cdots+x_{m} F_{m}>0
\end{array}
$$

siendo esta una desigualdad estricta definida positiva, aplicable también para una desigualdad no estricta, es decir, $F(x) \geq 0$, o para desigualdades estricta definida negativa $F(x)<0$ o desigualdades no estrictas $F(x) \leq 0$, donde:

- $F(x)$ es una función afín del vector real $x=\left[\begin{array}{lll}x_{1} & x_{2} \ldots x_{m}\end{array}\right]^{T}$

- $F_{0}, F_{1}, \ldots, F_{m}$, son matrices simétricas reales, es decir $F_{m}=F_{m}^{*}$ y se requiere que $F_{0} \in \mathbb{R}^{m x m}$ para que el LMI exista.

- $x_{i} \mid i \in\{1, \ldots, m\}$ es el vector de las variables de decisión

Algunas de sus principales propiedades son, [Scherer and Weiland, 2004]:

I. La LMI $F(x)<0$ define restricciones convexas en $x$, es decir, el conjunto de soluciones $F(x)<0$ es convexo. Si existe un sistema de LMI formado por un conjunto finito de LMI $F_{1}(x)<0, \ldots, F_{k}(x)<0$ el conjunto de todas las $x$ que satisfacen la desigualdad es convexa.

II. Las restricciones combinadas en ecuaciones del tipo $F(x)<0 \quad \mid \quad x \in \mathcal{M}$ donde $\mathcal{M}$ es un conjunto afin en $\mathbb{R}^{n}$ puede ser reescrito en la forma de un LMI simple $\hat{F}(\hat{x})<0$, donde la dimensión $\hat{n}$ de $\hat{x}$ debe ser al menos igual a la dimensión $n$ de $x$.

III. Es posible la transformación de una desigualdad no lineal en una desigualdad lineal empleando el complemento Schur: Sea una función afin $F: \mathbb{X} \rightarrow \mathbb{S}$ la cuál es particionada tal que:

$$
F(x)=\left[\begin{array}{ll}
F_{11}(x) & F_{12}(x) \\
F_{21}(x) & F_{22}(x)
\end{array}\right]
$$

donde $F_{11}(x)$ es cuadrada. Así, las siguientes expresiones son similares:

- a. $F(x)<0$

- b.

- c.

$$
\left\{\begin{array}{l}
F_{11}(x)<0 \\
F_{22}(x)-F_{21}(x)\left[F_{11}(x)\right]^{-1} F_{12}(x)<0
\end{array}\right.
$$

$$
\left\{\begin{array}{l}
F_{22}(x)<0 \\
F_{11}(x)-F_{12}(x)\left[F_{22}(x)\right]^{-1} F_{21}(x)<0
\end{array}\right.
$$

como se observa, estas dos últimas restricciones son no lineales respecto a $x$ y pueden convertirse en LMI.

Dado que la LMI $F(x)<0$ define una restricción convexa en la variable $x$, los problemas de optimización que involucren la minimización (o maximización) de una función de coste pertenecen a la clase de problemas de optimización convexa. Supongamos que $F: \mathbb{X} \rightarrow \mathbb{S}$ es afin, los dos problemas genéricos relacionados al estudio de LMI serán: 
1. Factibilidad. El LMI $F(x)<0$ es factible si existe un elemento $x \in \mathbb{X}$, en caso contrario se dice que no es factible

2. Optimización. Sea una función objetivo $f: \lambda \rightarrow \mathbb{R}$ donde $\lambda=x \mid F(x)<0$ el problema de determinar $V_{o p t}=\inf _{x \in \lambda} f(x)$ se denomina problema de optimización con restricciones LMI, este problema involucra encontrar la solución más óptima $\left(V_{o p t}<f(x)<V_{o p t}+\epsilon\right)$ o el cálculo de soluciones óptimas $\left(V_{\text {opt }}=f\left(x_{\text {opt }}\right)\right)$

Uno de los aspectos a considerar en el uso de LMI en sistemas lineales es que la ubicación de los polos garanticen la estabilidad cuadrática del sistema que se asegura mediante una tasa de decaimiento exponencial $\|x(t)\| \leq$ $M\left\|x_{o}\right\| e^{\alpha\left(t-t_{o}\right)}$ [Chilali et al., 1999], por ello se definen las regiones LMI: Para una matriz real simétrica $X \in \mathbb{R}^{2 n x 2 n}$ el conjunto de números complejos $z \in \mathbb{C}$ es una región LMI, [Guerra, 2006].

Las regiones más comunes son:

- Amortiguamiento garantizado: $\mathbb{R}(z)<-\alpha, \alpha>0$

- Círculo centrado en 0: $|z|<0$

- Ranura vertical: $\alpha_{1}<\mathbb{R}<\alpha_{2}<0$

- Cono centrado en el origen: $\mathbb{R}(z) \tan \theta<-|\operatorname{Im}(z)|, \theta \in[0, \phi / 2]$

Las aplicaciones de LMI en control, principalmente en la síntesis de controladores, se ha extendido en últimas fechas, algunas de estas aplicaciones se detallan a continuación:

1. Estabilidad. Consideremos un sistemas lineal autónomo definido por $\dot{x}=A x \quad \mid \quad A \in \mathbb{R}^{n x n}$. Lyapunov establece que este sistema es asintóticamente estable si y solo si $X=X^{T}$ tal que $X>0$ y $A^{T} X+X A<0$ si la función $V(x)=x^{T} X x$ es una función de Lyapunov, entonces la estabilidad asintótica es equivalente a la factibilidad del LMI:

$$
\left[\begin{array}{cc}
-X & 0 \\
0 & A^{T} X+X A
\end{array}\right]<0
$$

2. Rechazo a perturbaciones en base a la norma $H_{\infty}$. Dado el sistema LTI

$$
\left\{\begin{array}{l}
\dot{x}=A x+B u+B_{i} w \\
y=C x
\end{array}\right.
$$

donde $w$ es el vector de perturbaciones, y su función de transferencia está dada por $G(s)=Y(s) / W(s)$, cuya condición de rechazo de perturbación se define por $\|G(s)\|_{\infty}<\gamma$ si para $\gamma<1$ existe $P>0$ tal que sea factible el LMI, [Perez et ${ }^{\sim}$ al.]

$$
\left[\begin{array}{cc}
A P+P A^{T}+\gamma^{T} B_{i} B_{i}^{T} & P C^{T} \\
C P & -I
\end{array}\right]<0
$$

3. Diseño de sistemas de control. El diseño de un sistema de control activo empleando LMI para el posicionamiento del actuador (considerando para ello las limitaciones de entrada del actuador y la ubicación de los sensores 
empleando la norma $H_{\infty}$ ), el diseño de un controlador retroalimentado robusto (empleando una tasa de decaimiento definida y salidas limtadas) y de un observador es abordado en [Brennan et ${ }^{\sim}$ al., 2006].

4. Sistemas borrosos. Otra de las aplicaciones de LMI de los cuales existen diversos desarrollos se encuentra en el diseño de sistemas borrosos. Por ejemplo Tanaka et ${ }^{\sim}$ al. [1998] presenta el diseño de controladores y observadores borrosos empleando procedimientos de diseño basados en LMI considerando las condiciones de estabilidad, tasa de decaimiento, restricciones en la entrada y salida del control

\subsubsection{Observador empleando LMI}

Un observador de estado es robusto si es insensible a la incertidumbre hasta cierto grado. Hay diversos tipos de incertidumbre, uno de ellos es la incertidumbre en el modelo. En Gu and Poon [2001] se da una solución determinística derivada del resultado del error entre el estado verdadero y el estado observado que converge a cero empleando un observador Luenberger con la ayuda de un término no lineal, el cuál es determinado empleando el teorema de estabilidad de Lyapunov.

Así para la dinámica de una planta lineal, donde las incertidumbres en el modelo son consideradas como la perturbaciones en la matriz de estado $A=A_{0}+\Delta_{A}$, el observador de estado es un observador Luenberger con un término adicional $\alpha$, el cuál es empleado para que el error de estado converga a cero, a pesar de las perturbaciones $\Delta_{A}$ :

$$
\begin{array}{r}
\dot{\hat{x}}=A_{0} \hat{x}+B u+H(y-\hat{y})+\alpha \\
\hat{y}=C \hat{x}+D u
\end{array}
$$

donde el error residual se define como $r=C e, e$ es el error de estado, las perturbaciones están limitadas tal que $\left\|\Delta_{A}\right\|_{2}<\delta$ y el error de estado converge a cero si:

$$
\alpha=\frac{\delta^{2} \hat{x}^{T} \hat{x}}{2 r^{T} r} P^{-1} C^{T} r
$$

el cuál es determinado empleando la función de energía definida positiva del teorema de estabilidad de Lyapunov, $\mathrm{P}$ es una matriz definida positiva $P>0$ y satisface la desigualdad matricial:

$$
\left(A_{0}-H C\right)^{T} P+P\left(A_{0}-H C\right)+2 P^{2}+\delta^{2} I<0
$$

donde $H$ es tal que el término $A_{0}-H C$ es estable.

El procedimiento de solución de inecuaciones empleando LMI es una herramienta poderosa en la teoría de control y sus aplicaciones, tales como en el diseño de observadores de modos deslizantes.

Por ejemplo en Choi and Ro [2005] una condición necesaria y suficiente es obtenida de la existencia de un observador de modos deslizantes garantizando un movimiento deslizante estable insensible a ciertas incertidumbres. Las 
ganancias de las matrices del observador son obtenidas empleando la solución de la existencia de las condiciones de LMI.

Por otra parte, en Nian et ${ }^{\sim}$ al. [2006] se desarrolla un observador adaptativo de flujo magnético de ganancia constante para la identificación de la velocidad en un motor de inducción. En este desarrollo la teoría de estabilidad de Lyapunov es empleada para el diseño del observador de flujo magnético y estimador de la velocidad, su estabilidad es garantizada por un LMI y la solución del mismo proporciona las ganancias constantes del observador.

El empleo de un observador Luenberger ampliado empleando un término no lineal adicional denominado $\alpha$ el cuál es calculado mediante el criterio de estabilidad de Lyapunov se reporta en Gu and Poon [2001]. Este parámetro considera las incertidumbres del modelo aplicado a un ejemplo de un horno de gas, cuyo modelo tiene dos entradas y dos salidas.

El uso de la teoría de estabilidad de Lyapunov en conjunto con la aproximación de LMI se emplean también en Lien [2004] donde se propone el diseño de un controlador basado en observador para una clase de sistema que tenga incertidumbre paramétrica. Las ganancias del controlador y del observador se establecen empleando LMI construyendo un observador lineal de orden completo para garantizar que el sistema controlado retroalimentado sea estable exponencialmente.

Dado que el LMI puede emplearse en sistemas no lineales, el desarrollo de control y observadores en esta área está en franco crecimiento. Por ejemplo, [Arcak and Kokotović, 1999] emplea un LMI para determinar la matriz de ganancia del observador que satisface ciertas condiciones para un sistema con no linealidades monótonas. Por su parte, [Howell and Hedrick, 2002] diseña la matriz de ganancia empleando optimización convexa con respecto a tres costos: el máximo valor singular de la matriz de ganancia, la tasa de decaimiento de la dinámica del error y la norma $H_{\infty}$ entre las perturbaciones y los errores de estimación.

Por su parte, Bara et ${ }^{\sim} a l$. [2001] determina la matriz de ganancia del observador, dependiente de sus parámetros basándose en el esquema de un observador Luenberger, cuyo error de estimación converge asintóticamente a cero independientemente de las condiciones iniciales, la entrada de control y las variaciones de los parámetros. Para su definición emplea una función cuadrática de Lyapunov y las condiciones de estabilidad son resueltas mediante la factibilidad de un LMI. Coutinho et $\sim$ al. [2005] obtiene por su parte una matriz de ganancia del observador para un sistema no lineal con incertidumbre paramétrica y perturbaciones delimitadas, esta ganancia minimiza los efectos de la incertidumbre y el error en la estimación resuelto mediante LMI. 


\subsubsection{Observador de intervalo}

El objetivo del observador de intervalo es generar un estimador de estados con estimación limitada relacionada con la incertidumbre en el modelo o en la medición, [Dochain, 2003]. El diseño se basa en sistemas cooperativos, los cuales son sistemas dinámicos en cuya matriz Jacobiana los elementos fuera de la diagonal principal son positivos

$$
\frac{\partial f_{i}(x, t)}{\partial x_{j}} \geq 0, \quad i \neq j, t \geq 0
$$

Si definimos a un intervalo, $[x]$, como un subconjunto de $\mathbb{R}$ y al límite inferior del intervalo $[x]$ por $\underline{x}$ y al límite superior del intervalo $[x]$ por $\bar{x}$. Así, si existe una pareja de sistemas cooperativos, [Walter and Keiffer, 2003]:

$$
\left\{\begin{array}{l}
\underline{\dot{x}}=\underline{\dot{\bar{x}}}=\underline{\bar{f}}(\underline{x}, \underline{p}, \bar{p}, \bar{p}, t) \\
\bar{p}, t)
\end{array}\right.
$$

donde $p$ es un vector de parámetros que solamente se conocen dentro del intervalo $[p]$, tal que en un instante de tiempo $t,\{f(x, p, t) \mid p \in[p]\}$ es la solución del sistema dinámico para un valor dado, y $\{f(x,[p], t) \mid \forall p \in[p]\}$ es el conjunto de todos los valores que son solución del sistema dinámico, tal que

$$
\underline{f}(\underline{x}, \underline{p}, \bar{p}, t) \leq f(x, p, t) \leq \bar{f}(\bar{x}, \underline{p}, \bar{p}, t) \quad \forall p \in[\underline{p}, \bar{p}], t \geq 0
$$

con condiciones iniciales $\left\{\underline{x}_{0}(\underline{p}, \bar{p}) \leq x_{0}(p) \leq \bar{x}_{0}(\underline{p}, \bar{p})\right\}$. Entonces, la solución del sistema dinámico satisface

$$
\underline{x}(t) \leq x(t) \leq \bar{x}(t)
$$

donde:

- $\underline{x}(t)=\underline{\varphi}(\underline{p}, \bar{p}, t)$ está asociado con $\left\{\underline{\dot{x}}=\underline{f}(\underline{x}, \underline{p}, \bar{p}, t), \underline{x}(0)=\underline{x}_{0}(\underline{p}, \bar{p})\right\}$

- $\bar{x}(t)=\overline{\bar{\varphi}}(\underline{p}, \bar{p}, t)$ está asociado con $\left\{\dot{\bar{x}}=\overline{\bar{f}}(\bar{x}, \underline{p}, \bar{p}, t), \bar{x}(0)=\bar{x}_{0}(\underline{p}, \bar{p})\right\}$

En [Gouzé et ${ }^{\sim}$ al., 2000] se presenta una aplicación de este tipo de observador aplicado a un sistema de tratamiento de aguas bajo el siguiente esquema. Sea el sistema:

$$
\left\{\begin{array}{l}
\dot{x}(t)=A x(t)+\phi(t, y(t)) \\
y(t)=C x(t)
\end{array}\right.
$$

El observador de intervalo se define como:

$$
\left\{\begin{array}{l}
\dot{\dot{x}}(t)=A \underline{x}(t)+\underline{\phi}(t, y(t))+K(C \underline{\bar{x}}(t)-y(t)) \\
\overline{\dot{x}}(t)=A \bar{x}(t)+\overline{\bar{\phi}}(t, y(t))+K(C \bar{x}(t)-y(t))
\end{array}\right.
$$

El error total será $e(t)=\bar{e}(t)-\underline{e}(t)$ donde: 
- Error superior:

$$
\left\{\begin{array}{l}
\bar{e}(t)=\bar{x}(t)-x(t) \\
\dot{\bar{e}}(t)=(A+K C) \bar{e}+\bar{b}(t) \\
\bar{b}(t)=\bar{\phi}(t, y(t))-\phi(t, y(t))
\end{array}\right.
$$

- Error inferior:

$$
\left\{\begin{array}{l}
\underline{e}(t)=x(t)-\underline{x}(t) \\
\underline{\dot{e}}(t)=(A+K C) \underline{e}+\underline{b}(t) \\
\underline{b}(t)=\phi(t, y(t))-\underline{\phi}(t, y(t))
\end{array}\right.
$$

El observador de intervalo explícitamente integra los límites de incertidumbre en las entradas del proceso y provee límites explícitos para la estimación de los estados. Los resultados pueden ser conservadores si los límites seleccionados son demasiados grandes para integrar las peores incertidumbres. En [Rapaport and Gouzé, 2003] se presenta una generalización a este tipo de observador, denominándolo observador paralelotópico el cuál tiene límites variantes en el tiempo para las variables de estado, bajo la hipótesis de que existen límites disponibles para los términos inciertos.

\subsubsection{Estimadores de Horizonte Móvil}

Varios autores han obtenido desarrollos basados en horizonte móvil para la estimación en línea de estados y parámetros, como extensiones de los algoritmos de estimación de mínimos cuadrados por lote [Schei and Johansen]. Estos algoritmos se basan en una ventana de datos finitos de las mediciones anteriores. Las variables de decisión en estos algoritmos se basan en parámetros y estados iniciales, que se supone constantes durante el horizonte temporal definido por la ventana de datos. Por lo tanto, se supone que los estados iniciales y los parámetros determinan por completo el valor de todos los futuros estados en el horizonte de predicción.

[D.G. ${ }^{R}$ Robertson and Rawlings, 1996] presentan un formulación más general del estimador de horizonte móvil, donde los estados y los parámetros, además de los resultados de proceso, se supone que son influidos por las perturbaciones estocásticas. Esto enfoque es, sin embargo, computacionalmente mucho más exigentes para una ventana de datos de largo. También dan un panorama informativo de horizonte móvil basados en diferentes los enfoques, y hacen comparaciones con los métodos recursivos como EKF.

El estimador de horizonte móvil (Moving Horizont Estimation, MHE) presenta algunas ventajas sobre estimadores clásico, como el filtro de Kalman extendido (EKF), como son la posibilidad de considerar las limitaciones físicas de los estados (por ejemplo, en el caso de los bioprocesos, las concentraciones son siempre mayores o iguales a cero) y el hecho de que en el horizonte considerado no se pierde ninguna información sobre la sistema no lineal. La 
principal desventaja es la necesidad de la resolución de un programa dinámico no lineal, [L. `S.Ferreira and Secchi, 2003].

Por su parte, [Gonzalo Valdez-Gonzalez and Zolezzi, 2006] presentan una estrategia aplicada al problema de estimación no lineal de estados en un motor de inducción (MI). Este método permite, en general, la estimación de variables de difícil acceso o que simplemente no se pueden medir, lo cual es posible a través de mediciones indirectas, considerando un modelo dinámico del proceso y un algoritmo de estimación basado en optimización no lineal. Esta estrategia de estimación de estados se denomina MHSE (Moving Horizon State Estimation) en un MI, El método consiste en la sustitución de un problema de estimación dinámica con un problema de optimización estática no-lineal. El criterio de definición para este tipo de problema es la suma del error cuadrático entre la entrada y la mediciones de la producción estimada por el modelo de proceso en un horizonte de tiempo predefinido.

La minimización de este criterio tiene por objeto determinar el estado estimado en el comienzo de la horizonte de tiempo, de tal manera que ello conduce a una reducción al mínimo entre mediciones observadas y la trayectoria prevista en el horizonte.

Los MHE relacionados con la solución de un problema de optimización dinámica se han propuesto para manejar las restricciones. Un tema crítico asociado con MHE, en particular para el sistema no lineal, es el coste computacional asociado con la solución del problema de optimización dinámica. En Mahadevan and III [2004], un enfoque basado en la estructura del modelo de proceso no lineal se propone para la solución de los problemas de optimización en MHE. El concepto de "flatness" diferencial, una característica de la estructura del modelo de proceso no lineal, ha sido explotados en el pasado para la optimización dinámica en EKF en el contexto del modelo de problema de control predictivo (el dual de la MHE no lineal). La idea se amplia para el control de los modelos de procesos no lineales que no son de manera diferente a través de una combinación del enfoque simultáneo y el enfoque basado en "flatness" (enfoque basado en la eliminación de entrada).

\subsubsection{Observadores multifrecuenciales}

En un ambiente típico de bioprocesos, es común que la medición de las variables fundamentales del proceso, como la concentración de la biomasa, no puedan medirse o se realicen en forma esporádica y/o irregular fuera de línea y con retardos de tiempo significativos. En ausencia de mediciones frecuentes de las variables esenciales, las estimaciones continuas de estas puede obtenerse de las mediciones frecuentes y/o esporádicas disponibles por un modelo del proceso bajo consideración.

Ejemplos de mediciones frecuentes (o "rápidas") son la temperatura, presión, oxígeno disuelto y densidad, y son instantáneas tomando en consideración que la medición es realizada y su valor está disponible en el mismo instante de 
muestreo.

Mediciones como el peso molecular promedio de polímeros (obtenido por medio de cromatografía), la medida de la concentración de metabolitos y la concentración de biomasa (aunque este puede realizarse en forma indirecta y en tiempo real pero con errores) se encuentran disponibles un tiempo posterior en el que se realizó el muestreo y/o en forma irregular, además de tener retardos de tiempo significativos, son ejemplos de mediciones esporádicas (o "lentas").

Los sistemas con mediciones multifrecuenciales pueden dividirse en dos categorías [Mutha et $\sim a l ., ~ 1997]$ :

- Sistemas con mediciones primarias y secundarias, donde las mediciones secundarias inferenciales (mediciones frecuentes) están disponibles más frecuentemente que las mediciones primarias y los estimadores de estados pueden hacerse observables usando las mediciones secundarias aún en la ausencia de mediciones primarias.

- Sistemas con mediciones frecuentes (rápidas) y esporádicas (lentas), en los cuales la estructura del modelo el proceso y, por lo tanto, el flujo de la información (la dirección de propagación) es tal que la observabilidad del estado completo no puede darse sin las mediciones lentas.

La estimación de variables multifrecuenciales permite usar las medidas disponibles esporádicamente en la estimación de estados y parámetros, principalmente para una mejora considerable en la exactitud de la estimación resultante.

El problema de la estimación de estados y parámetros multifrecuencial ha recibido atención, con énfasis en la extensión del filtro Kalman extendido para procesos con medidas retardas y multifrecuenciales.

En estos estimadores, el vector de medición se expande para incluir a las medidas fuera de línea, cuando estén disponibles. Para resolver el problema de tiempo de retardo debido causado por los análisis de laboratorio, las estimaciones son recalculadas del momento de la medición al tiempo presente, [Dondo, 2004]

Gudi and Shah [1993] plantea que para generar óptimamente estimaciones de estado filtradas, considerando el mejor modelo disponible, se obtiene cuando las mediciones son logradas a diferentes razones de muestreo.

Las ecuaciones globales del filtro Kalman en forma multifrecuencial son iguales que para el filtro clásico, pero con periodicidad en las matrices de covarianzas de las ganancias Kalman, mediciones y proceso. Este modelo es adaptado a las características variantes en el tiempo del proceso usando la máxima probabilidad, y empleando una estrategia de estimación secuencial de parámetros.

Por su parte, en Z.I.T.A. Soons and van Boxtela [2007] se presenta un observador empleado para el monitoreo de la producción de un fármaco. Este observador está desarrollado con EKF y consiste en tres parte: Un estimador de 
medidas frecuentes (para la tasa de crecimiento y biomasa), un estimador de medidas esporádicas (activado por las medidas fuera de línea para la biomasa y el coeficiente de transporte de oxígeno, $k_{L} a$ ) y una etapa de aprendizaje, para mejorar la relación entre la biomasa y el coeficiente de transporte de oxígeno.

En fermentaciones típicas, la razón de crecimiento específica es variante en el tiempo, debido a su dependencia de las concentraciones de los nutrientes que varían con el tiempo. Por lo tanto cuando las actividades de mantenimiento son despreciables o constantes, es posible usar un filtro Kalman extendido (EKF) para la estimación simultánea de la biomasa como el estado y la razón de crecimiento específica como el parámetro [Gudi et ${ }^{\sim} a l ., 1994$ ].

En el caso de estudio, se define el "mayor" instante de muestreo cuando se cuenta con las mediciones de la biomasa (medición esporádica) y el dióxido de carbono, CER (medición frecuente), y el "menor"instante de muestreo ocurre cuando solo se cuenta con la medición del CER. Estos eventos ocurren con un tiempo de muestreo base de 20 minutos y el instante mayor ocurre en nueve instantes de muestreo (3 horas).

La insuficiencia del EKF para varios procesos no lineales y los avances en el diseño de observadores no lineales han motivado el desarrollo y uso de observadores de estado multifrecuenciales no lineales.

El desarrollo de un método de diseño para un observador no lineal de ganancias variantes se aplica a un reactor exotérmico clásico con múltiples estados estables [M.Soroush and Valluri, 1996], y a un reactor de polimerización [Soroush and Tatiraju, 1997].

Se presentan ademas otros casos donde se extiende para ser usado en procesos no lineales con mediciones multifrecuenciales y retardadas, como un reactor bioquímico [Tatiraju et ${ }^{\sim a l ., ~ 1998],[T a t i r a j u ~ e t ~}{ }^{\sim}$ al., 1999a].

En este caso, los estados (la concentración de células viables, células totales, glucosa, glutamina y anticuerpos monoclonales (MAb)), se estiman de la mediciones frecuentes de la razón de suministro de oxígeno (OUR) y de mediciones esporádicas y retardadas de las concentraciones de las cinco variables de estado y un reactor de polimerización [Tatiraju et ${ }^{\sim} a l ., 1999 \mathrm{~b}$ ].

Una de las diferencias importantes entre estos desarrollos es que un EKF es sintonizado ajustando las matrices de covarianzas del ruido en la señal y el error de estimación inicial, mientras que el observador de estado multifrecuencial es sintonizado ajustando las matrices de ganancia $K$, asociada al error en las medidas "rápidas" y la matriz de ganancias $L$, asociada con el error en las medidas "lentas".

Los componentes de la ganancia del estimador multifrecuencial son ajustados de tal manera que los valores propios de la matriz Jacobiana de las dinámicas de error observadas estén localmente en el plano izquierdo.

Otra diferencia importante se relaciona con el gasto computacional ya que para un proceso con $n$ variables de estado y $p$ salidas rápidas medibles, el observador de estado será del orden $n-p$, mientras que para el mismo proceso 
un EKF estándard sería del orden $n(n+1)$

La combinación del estimador de estado no lineal multifrecuencial con un controlador de estado retroalimentado se reporta aplicándolo a un reactor de polimerización, en donde la calidad del producto usualmente no es observable de las medidas frecuentes con retardo libre, motivando con ello la aplicación del controlador multifrecuencial [Zambare and Soroush, 1999], [Zambare etªl., 2002].

\subsection{Discusión}

En este capítulo se han presentado los modelos generales para el modelado matemáticos de los bioprocesos, así como los observadores de estados, analizándose las principales metodologías para el diseño de observadores: exponenciales, asintótico y otros esquemas emergentes que se han sido reportados en diversos foros académicos.

Esta revisión permite conocer el estado del arte necesario para poder cumplir con el primer objetivo particular planteado para esta tesis: Analizar diversas propuestas de algoritmos de observadores de estados y parámetros aplicados a los bioprocesos y aquellos que puedan aplicarse a ellos.

En el siguiente capítulo se desarrolla un algoritmo de estimación basado en un observador Luenberger cuya matriz de ganancia sera determinada considerando las incertidumbres del modelo, debido a los problemas en el conocimiento de la planta, mejorando su convergencia respecto a los desarrollos clásicos. 


\title{
3 Observador para bioprocesos empleando LMI
}

\author{
Nuestra recompensa se encuentra en el esfuerzo y no con el resultado. Un \\ esfuerzo total es una victoria completa
}

Gandhi

\subsection{Introducción}

Los modelos de bioprocesos han tenido un gran desarrollo en las últimas décadas. El grado de aproximación depende de factores como el conocimiento del comportamiento de la reacción, los datos requeridos para la construcción y validación del modelo, requerimientos computacionales y el uso que se le dará al modelo.

Debido a estos y otros factores, la incertidumbre en el modelo está presente, dado que el conocimiento exacto de los parámetros del mismo no llega a ser preciso, teniendo además la posibilidad de incertidumbre en la medida, producida por ruido en la medición. También, la ubicación física de un sensor en el bioreactor produce cambios en la observabilidad del modelo, [Tali-Maamar and Babary, 1994]

El filtro Kalman considera esta incertidumbre como ruido blanco asociado con el modelo y la medida, para optimizar la estimación. Por otra parte, si se conocen los límites del modelo dinámico general, tal que se pueda formar un politopo que incluya la familia de todas las posibles situaciones, como la incertidumbre en el modelo, las ganancias del observador garantizarán la convergencia global.

Estas ganancias serán válidas en el universo formado por el politopo. Entonces podremos contar con un estimador que garantice su convergencia y estabilidad en ese espacio.

El problema a investigar en este capítulo se centra en la estimación de variables no medidas para sistemas dinámicos biológicos con incertidumbre. Por incertidumbre entenderemos que alguna parte del modelo no se conoce con precisión o que existe algún componente de ruido. Aún cuando este problema es clásico, se propone realizar la estimación empleando métodos de análisis novedosos.

Así, en este capítulo se presenta el desarrollo de un observador no lineal empleando el procedimiento LMI para la definición de la ganancia del mismo. 
Para ello se define un politopo cuyos vértices están definidos por los intervalos de operación del bioproceso. El modelo matemático del error es resuelto empleando el teorema del valor medio diferencial, en lugar de linealizar alrededor del origen.

\subsection{Análisis del modelo}

En el monitoreo y control de procesos bioquímicos están presentes dos problemas típicos [Dochain, 2001]: por un lado las dinámicas son en general complejas, con un gran número de variables y no lineales. Esto dificulta determinar todos los factores que influyen en la cinética del proceso y tener datos confiables para la calibración de los parámetros del modelo.

El segundo problema está relacionado con la disponibilidad de instrumentos que permitan la medición en línea de todas las variables claves del proceso (reactantes, productos y biomasa), las cuales son requeridos para el control de calidad.

El diseño de un observador permite solventar el segundo problema. Para resolver el problema de las incertidumbres en el modelo, podemos incluirlas en el diseño del observador.

Para el modelo dinámico general, la dinámica del error están definida por la siguiente ecuación diferencial:

$$
\frac{d e}{d t}=K[\varphi(\hat{\xi}+e)-\varphi(\hat{\xi})]-D e-\gamma L e
$$

La tasa de reacción $\varphi(\xi)$ es proporcional a las concentraciones de los reactantes $\xi(t)$ involucrados en la reacción y a la tasa específica de reacción. La aproximación más general en el modelado de biorreactores consiste en adoptar una estructura analítica particular (ver la tabla 2.1) para cada tasa de reacción específica, y calibrar sus coeficientes internos de acuerdo a datos experimentales. Todo esto representa incertidumbre en el modelo.

Dado que los bioprocesos son sistemas que cuentan con entradas y estados limitados, podemos definir los límites en los cuales estos parámetros operan y en consecuencia se definen los límites de las tasas de reacción. Así de acuerdo a sus características físicas, [Bastin and Dochain, 1990]:

- La tasa de disolución $\mathrm{D}(\mathrm{t})$ es estrictamente positiva y está limitado en su valor mínimo:

$D(t) \geq 0 \quad \forall t$

- Las tasas de alimentación están limitas y, dado que $F_{i}=D(t) S_{i n, i}(t)$, entonces se asume la siguiente limitación:

$0 \leq S_{i n, i}(t) \leq S_{\max } \quad \forall i, \quad \forall t$

- Cada reacción involucra al menos un reactante $\xi_{n}$, el cuál no es catalizador ni autocatalizador. 
- Los valores iniciales de las variables de estado $\xi_{i}(t)$ tienen limites superiores:

$a_{n} \xi_{n}(0)+\sum_{i \in I} \xi_{i}(0) \leq\left(a_{n}+q\right) S_{\max }$

donde :

$$
a_{n}=\operatorname{máx}_{j \in J} \frac{\sum_{i \in I} \bar{k}_{i j}}{-\bar{k}_{n j}} \geq 0
$$

$\bar{k}_{i j}$ es el coeficiente $(\mathrm{i}, \mathrm{j})$ de la matriz $\mathrm{K}$.

- $J=\left(m_{1}, \ldots, m_{p}\right)$ : conjuntos de índice de las reacciones las cuales involucra a $\xi_{n}$ como un reactante.

- $I=\left(n_{1}, \ldots, n_{q}\right)$ : conjuntos de índice de los componentes (excluyendo a $\xi_{n}$ ) involucrado en las reacciones que tienen un índice $m_{j} \in J$

Así, bajo estas condiciones, las variables de estado $\xi_{i}(t)$ del modelo dinámico general, ecuación 2.2 son positivos y limitados para toda $t$, [Johnson, 1987]:

$$
0 \leq \xi_{i}(t) \leq \operatorname{máx}\left\{1, \frac{1}{a_{n}}\right\}\left(a_{n}+q\right) S_{\max }
$$

Si $\bar{\xi}=\operatorname{máx}\{\xi\}$ y $\underline{\xi}=\operatorname{mín}\{\xi\}$, entonces podemos definir los límites de las tasas de reacción y en consecuencia de la ecuación dinámica del error:

$$
\begin{aligned}
& \frac{d \bar{e}}{d t}=K[\bar{\varphi}(\hat{\xi}+e)-\bar{\varphi}(\hat{\xi})]-D e-\gamma L e \\
& \frac{d \underline{e}}{d t}=K[\underline{\varphi}(\hat{\xi}+e)-\underline{\varphi}(\hat{\xi})]-D e-\gamma L e
\end{aligned}
$$

donde las tasas de reacción estarán acotadas a un valor máximo $(\bar{\varphi})$ y un valor mínimo $(\underline{\varphi})$ que dependerán de las regiones definidas por los valores acotados de los estados del modelo:

$$
\begin{aligned}
& \bar{\varphi}(\xi)=\operatorname{máx}\{\varphi(\bar{\xi}, \underline{\xi})\} \\
& \underline{\varphi}(\xi)=\operatorname{mín}\{\varphi(\bar{\xi}, \underline{\xi})\}
\end{aligned}
$$

Estos límites representan entonces un politopo. Un politopo representa una región en el espacio limitado por $n$ dimensiones. La incertidumbre politópica es la representación de la incertidumbre paramétrica que afecta a un sistema. Una clasificación de politopos polinomiales cuyos vértices se encuentran compuestos por polinomios, se define como [Henrion et ${ }^{\sim}$ al., 2001]:

- Intervalos polinomiales, donde cada coeficiente del polinomio varía independientemente en un intervalo específico. 
- Politopo polinomial, también referido como familia polinomial afín, la cuál son combinaciones lineales de un conjunto de polinomios dados. Estas familias tienen conjuntos de valores los cuales son polígonos convexos en el plano complejo.

- Familia polinomial multilineal, donde cada coeficiente de un polinomio es una función multilineal de los parámetros con incertidumbre. Estas familias son politopos de matrices polinomiales cuyas entradas son intervalos polinomiales independientes.

- Politopos de matrices polinomiales, los cuales son combinaciones lineales de un conjunto de matrices polinomiales dadas. En estas familias los coeficientes de los determinantes de una matriz polinomial en el politopo son polinomios multivariables.

Entonces, si definimos:

$$
\mathcal{A}_{i}=\left[\begin{array}{llll}
A_{0} & A_{1} & \ldots & A_{n}
\end{array}\right]
$$

como las matrices constantes asociadas a la matriz polinomial

$$
A_{i}(s)=A_{0}+s A_{1}+\cdots+s^{n} A_{n}
$$

de dimensión $n$, el politopo matricial polinomial $\mathcal{P}$ es estable si existen matrices $X_{1}, X_{2}, \ldots, X_{n}$ que resuelvan el LMI, [Henrion et ${ }^{\sim}$ al., 2001]:

$$
\left[\begin{array}{cc}
-X_{i} & 0 \\
0 & \mathcal{A}_{i}^{T} X_{i}+X_{i} \mathcal{A}_{i}
\end{array}\right]<0
$$

Dado que el procedimiento LMI se basa en sistemas lineales, y siendo los bioprocesos sistemas dinámicos no lineales, se requiere acotar el modelo dinámico general no lineal por un politopo formado por modelos lineales, para lo cuál se pueden emplear diversos métodos.

Uno de estos métodos consiste en usar un cambio no lineal de coordenadas para llevar el sistema original a un sistema lineal o pseudo-lineal.

Esta aproximación simplifica el diseño del observador. Sin embargo se requiere que diversas condiciones sean cubiertas y es válido solo para una clase de sistemas muy particular.

La alternativa propuesta en este capítulo consiste en emplear el Teorema del Valor Medio Diferencial para escribir la dinámica del error del observador cono un sistema lineal. Realizar el análisis de estabilidad empleando una función cuadrática de Lyapunov, y garantizar la convergencia global de la ganancia del observador empleando un procedimiento basado en el uso de LMI.

\subsection{Teorema del valor medio diferencial}

El modelo tangencial linealizado del error de observación se emplea para la linealización del modelo de bioprocesos. Sin embargo, dado que se realiza 
dicha linealización alrededor del punto de equilibrio, se requiere que el sistema tenga observabilidad local en dicho punto.

Un método alternativo es la aplicación del teorema del valor medio diferencial, el cuál permite acotar la dinámica del error de observación mediante un conjunto de modelos lineales.

El teorema de valor medio para funciones reales diferenciables establece que, [Antczak, 2005], dada una función $f: C \rightarrow \mathbb{R}, C \subset \mathbb{R}^{n}$, existe un punto $c$ entre $a$ y $b$ tal que:

$$
f(b)-f(a)=(b-a)^{T} \nabla f(c)
$$

donde $\nabla f(c)$ denota el gradiente de $f$ en $c$.

Teorema: Sea $(x, y) \in \mathbb{R}^{n}$, definimos por $C o(x, y)$ la envolvente convexa del conjunto $x, y$ :

$$
C o(x, y)=\{\lambda x+(1-\lambda) y, \lambda \in[0,1]\}
$$

El Teorema del Valor Medio Diferencial ('Differential Mean Value Theorem, $D M V T^{\prime}$ ') en $\mathbb{R}^{n}$ se define como:

Teorema: Sea $f: \mathbb{R}^{n} \rightarrow \mathbb{R}^{q}, a, b \in \mathbb{R}^{n}$. Considerando que $f$ es diferenciable en $C o(a, b)$. Entonces existe una constante $c \in C o(a, b), c \neq a, c \neq b$ tal que:

$$
f(a)-f(b)=f^{\prime}(c)(a-b)
$$

donde

$$
f^{\prime}=\left[\frac{\partial f}{\partial x_{1}} \ldots \frac{\partial f}{\partial x_{n}}\right]
$$

Dado que el DMVT no se aplica a una función vector, Zemouche et ${ }^{\sim}$ al. [2005] propone lo siguiente:

Sea $f: \mathbb{R}^{n} \rightarrow \mathbb{R}^{q}$ una función vector. Si $f(x)=\left[f_{1}(x), \ldots, f_{q}(x)\right]^{T}$, donde $f_{i}: \mathbb{R}^{n} \rightarrow \mathbb{R}^{q}$ es el $i$-esimo componente de $f$, entonces:

$$
f(x)=\sum_{i=1}^{q} \epsilon_{q}(i) f_{i}(x)
$$

donde:

$$
\Xi_{q}=\left\{\epsilon_{q}(i) \mid \epsilon_{q}(i)=(0, \ldots, 0,1,0, \ldots, 0)^{T}, i=1, \ldots, q\right\}
$$

es la base canónica del espacio vectorial $\mathbb{R}^{q}, \forall q>1$ 
Definición: Sea $f: \mathbb{R}^{n} \rightarrow \mathbb{R},(a, b) \in \mathbb{R}^{n}$. Se asume que $f$ es diferenciable en $C o(a, b)$. Entonces, hay vectores constantes $c_{1}, \ldots, c_{q} \in C o(a, b), c_{i} \neq$ $a, c_{i} \neq b$, para $i=1, \ldots, q$ tal que:

$$
f(a)-f(b)=\left(\sum_{i, j=1}^{q, n} \epsilon_{q}(i) \epsilon_{n}^{T}(j) \frac{\partial f_{i}}{\partial x_{j}}\left(c_{i}\right)\right)(a-b)
$$

\subsubsection{Aplicación de DMVT al modelo dinámico general}

El concepto anterior podemos aplicarlo a la ecuación que define la dinámica del error de observación, ecuación 2.16 :

$$
\frac{d e}{d t}=K[\varphi(\xi)-\varphi(\hat{\xi})]-D e(t)-\gamma L e(t)
$$

donde $e(t)=\xi-\hat{\xi}$, para la resolución de $[\varphi(\xi)-\varphi(\hat{\xi})]$, obteniendo con ello el politopo que define al modelo.

Así aplicamos el concepto DMVT. Existe $\xi^{*} \in C o(\xi, \hat{\xi})$ es decir, existe un valor del estado del bioproceso comprendido dentro de los límites marcados por el valor real y el valor estimado, tal que:

$$
\varphi(\xi)-\varphi(\hat{\xi})=\frac{\partial \varphi_{m}\left(\xi^{*}\right)}{\partial \xi_{n}}(\xi-\hat{\xi})=\left(\sum_{i, j=1}^{m, n} \epsilon_{m}(i) \epsilon_{n}^{T}(j) \frac{\partial \varphi_{i}}{\partial \xi_{j}}\left(\xi^{*}\right)\right) e(t)
$$

donde $\xi_{j},\{j=1, \ldots, n\}$ son los estados del modelo y $\varphi_{i},\{i=1, \ldots, m\}$ son los elementos del vector de tasas de reacción. Si definimos:

$$
\begin{aligned}
h_{i j}(t) & =\frac{\partial \varphi_{i}}{\partial \xi_{j}}\left(\xi^{*}\right) \\
h(t) & =\left(h_{11}(t), \ldots, h_{m n}(t)\right)
\end{aligned}
$$

la dinámica del error de observación para un bioproceso será:

$$
\frac{d e}{d t}=\left(\sum_{i, j=1}^{m, n} h_{i j}(t) K \epsilon_{m}(i) \epsilon_{n}^{T}(j)-D-\gamma L\right) e(t)
$$

donde definimos:

$$
\mathcal{A}(h(t))=\sum_{i, j=1}^{m, n} h_{i j}(t) K \epsilon_{m}(i) \epsilon_{n}^{T}(j)-D I_{n}
$$

Entonces

$$
\dot{e}(t)=(\mathcal{A}(h(t))-\gamma L) e(t)
$$




\subsection{Análisis de convergencia}

Para estudiar la convergencia exponencial del error del observador, consideremos la función cuadrática de Lyapunov:

$$
V(t)=V(e)=e^{T} P e
$$

donde $\mathrm{P}$ es una matriz simétrica, $P=P^{T}>0$. El error converge exponencialmente a cero si $V(e)>0, \dot{V}(e)<0$ para todo $e(t) \neq 0$, y entonces el sistema es asintóticamente estable. Empleando la ecuación de la dinámica del error:

$$
\begin{aligned}
\dot{e} & =(\mathcal{A}(h)-\gamma L) e \\
\dot{V}(e) & =e^{T}(\mathcal{A}(h)-\gamma L)^{T} P e+e^{T} P(\mathcal{A}(h)-\gamma L) e \\
\dot{V}(e) & =e^{T}\left(\mathcal{A}(h)^{T} P-L^{T} \gamma^{T} P+P \mathcal{A}(h)-P \gamma L\right) e
\end{aligned}
$$

La condición $V(e)>0$ se satisface ya que la matriz $\mathrm{P}$ es definida positiva. La segunda condición, $\dot{V}(e)<0$ se satisface si:

$$
\mathcal{M}(h)=\mathcal{A}(h)^{T} P-L^{T} \gamma^{T} P+P \mathcal{A}(h)-P \gamma L<0 \quad \forall h(t) \in \mathcal{H}_{q, n}
$$

Las tasas de reacción y sus derivadas $\left(h_{i j}(t)\right)$ están limitadas tal que:

$$
\operatorname{máx}_{t}\left|h_{i j}(t)\right|<+\infty \quad\{\forall i=1, \ldots, m \mid \forall j=1, \ldots, n\}
$$

Entonces el vector $h(t)$ está limitado en el dominio $\mathcal{H}_{m, n}$, cuyos $2^{m n}$ vértices se definen por $\mathcal{V}_{\mathcal{H}_{m, n}}=\alpha$, donde $\alpha$ es un valor comprendido dentro de los límites definido para el intervalo $\left[h_{i j}\right]$ :

$$
\alpha=\left(\alpha_{11}, \ldots, \alpha_{1 n}, \ldots, \alpha_{m n}\right) \quad \mid \alpha_{i j} \in\left\{\underline{h}_{i j}, \bar{h}_{i j}\right\}
$$

y donde los límites superior e inferior del intervalo se definen como:

$$
\begin{gathered}
\bar{h}_{i j}=\operatorname{máx}_{t}\left(h_{i j}(t)\right)=\frac{\partial \overline{\varphi_{i}}}{\partial \xi_{j}}\left(\xi^{*}\right) \\
\underline{h}_{i j}=\operatorname{mín}_{t}\left(h_{i j}(t)\right)=\frac{\partial \underline{\varphi_{i}}}{\partial \xi_{j}}\left(\xi^{*}\right)
\end{gathered}
$$

Es decir, el intervalo $\alpha_{i j}$ define los límites inferior y superior que se tendrá cuando se consideren los valores máximos y mínimos que se tendrán en la definición de las tasas de reacción al sustituir los valores esperados de cada uno de los parámetros.

Dado que la matriz $\mathcal{M}(h)$ es afín en $h(t)$, entonces, usando el principio de convexidad, se deduce que $\dot{V}(e)<0$ si se satisface la condición $\mathcal{M}(\alpha)<0$, es decir:

$$
\mathcal{A}(\alpha)^{T} P-L^{T} \gamma^{T} P+P \mathcal{A}(\alpha)-P \gamma L<0 \quad \forall \alpha \in\left[h_{i j}\right]
$$

Así, dadas las matrices polinomiales cuadradas $\mathcal{A}\left(\alpha_{11}\right), \ldots, \mathcal{A}\left(\alpha_{m n}\right)$ se define el politopo $\mathcal{P}$ como el conjunto de todas las matrices polinomiales $\mathcal{A}(\alpha)$. 
Siendo $\mathcal{P}$ estable, todas las matrices polinomiales serán estables, y el error del observador $e(t)$ convergerá exponencialmente hacia cero si existen las matrices $P=P^{T}>0$ y $R=\gamma^{T} P$, tal que el LMI:

$$
\mathcal{A}(\alpha)^{T} P-L^{T} R+P \mathcal{A}(\alpha)-R^{T} L<0 \quad \forall \alpha \in\left[h_{i j}\right]
$$

sea factible. Cuando sea así, la ganancia del observador se determina mediante $\gamma=P^{-1} R^{T}$

\subsection{Análisis del Algoritmo del Observador}

El observador propuesto es esencialmente el esquema de un observador Luenberger extendido, siendo su principal diferencia que no emplea la linealización tangencial del modelo, si no el concepto del Valor Medio Diferencial. Este concepto define un valor entre el estado real y el estimado, alrededor del cuál se definen las cotas de las tasas de reacción y de sus derivadas para establecer el politopo en el cuál se trabaja.

Sin embargo, la definición de las ganancias del observador, al realizarse mediante LMI, permite considerar la incertidumbre en el modelo. Es esta la característica principal del algoritmo, ya que las incertidumbres que se tengan al modelar el bioproceso pueden considerarse cuando se defina el valor de la ganancia, considerando para ello que se cuenta con un modelo de bioreactor bien definido y conocido.

Estas incertidumbres pueden considerar también el ruido en la medida, siendo entonces estable el observador ante la presencia de cierto nivel de ruido.

La matriz $\mathcal{A}$ representa el comportamiento dinámico del error de estimación, y es una matriz cuadrada cuya dimensión depende del número de estado, tal que $\mathcal{A} \in \mathbb{R}^{n x n}$. Esta matriz y sus derivadas están definidas y sus valores propios se ubican en el plano izquierdo, cumpliendo con los requerimientos definidos para el observador Luenberger.

Cada uno de los términos de la matriz $\mathcal{A}$ está conformado por las Jacobianas de la tasa reacción respecto a cada uno de los estados del sistema, como se define en la ecuación 3.22. Para explicar la estructura de esta matriz, emplearemos unos ejemplos demostrativos.

Ejemplo 3.1 Sea el bioproceso definido por:

$$
\left[\begin{array}{c}
\hat{X} \\
\hat{S}
\end{array}\right]=\left[\begin{array}{c}
1 \\
-k
\end{array}\right] \varphi-D\left[\begin{array}{c}
X \\
S
\end{array}\right]-\left[\begin{array}{c}
0 \\
D S_{i}
\end{array}\right]
$$

este sistema está compuesto de una tasa de reacción y dos estados tal que $m=1 \quad y \quad n=2$

Así la matriz $\mathcal{A}$ queda definida como:

$$
\mathcal{A}(h)=\left[h_{11} K \epsilon_{1}(1) \epsilon_{2}(1)^{T}+h_{12} K \epsilon_{1}(1) \epsilon_{2}(2)^{T}\right]-D I_{2}
$$


donde $\epsilon_{m}(i)=\epsilon_{1}(1)=1, \epsilon_{2}(1)^{T}=\left[\begin{array}{ll}1 & 0\end{array}\right], \mathrm{y} \epsilon_{2}(2)^{T}=\left[\begin{array}{ll}0 & 1\end{array}\right], h_{11}=\frac{\partial \varphi}{\partial X} \mathrm{y}$ $h_{12}=\frac{\partial \varphi}{\partial S}$, así:

$$
\mathcal{A}(h)=\left[\begin{array}{cc}
\frac{\partial \varphi}{\partial X}-D & \frac{\partial \varphi}{\partial S} \\
-\frac{\partial \varphi}{\partial X} k_{1} & -\frac{\partial \varphi}{\partial S} k_{1}-D
\end{array}\right]
$$

Como se observa, el limite de la matriz $\mathcal{A}$, si algún término $h_{i j} \rightarrow 0$, será una matriz diagonal teniendo en la diagonal principal a la tasa de disolución $D$, de manera que la convergencia del observador dependerá de las condiciones de operación si el substrato tiende a valores muy pequeños.

Los vértices del politopo del dominio $\mathcal{H}_{m, n}$ dentro del cuál el parámetro $h_{i j}$ está limitado, se definen dentro del intervalo delimitado por $\left\{\underline{h_{i j}, \overline{h_{i j}}}\right\}$ :

$$
\begin{array}{r}
\mathcal{A}(\alpha)=\mathcal{A}\left(\alpha_{11}, \alpha_{12}\right) \\
\alpha_{11} \in \underline{h}_{11}, \bar{h}_{11} \\
\alpha_{12} \in \underline{h}_{12}, \bar{h}_{12}
\end{array}
$$

teniendo entonces $2^{n}$ vértices que definen el espacio en el cuál los valores calculados de la matriz de ganancia satisface las condiciones de estabilidad. La estructura de la matriz de acuerdo a las Jacobianas, se ejemplifican a continuación empleando tres modelos de la velocidad específica de crecimiento $\mu$, la cuál define a la tasa de reacción $\varphi=\mu X$.

a). Si $\mu$ se define de acuerdo a la ecuación de Monod (la cuál es una función monótona y depende únicamente del sustrato):

$$
\mu=\frac{\mu_{m} S}{K_{s}+S}
$$

entonces la matriz $\mathcal{A}$ especificamente para este caso se define como:

$$
\mathcal{A}(h)=\left[\begin{array}{cc}
\frac{\mu_{m} S}{K s+S}-D & \frac{\mu_{m} X}{K s+S}-\frac{\mu_{m} S X}{(K s+S)^{2}} \\
-\frac{\mu_{m} S}{K s+S} k_{1}-\left(\frac{\mu_{m} X}{K s+S}-\frac{\mu_{m} S X}{(K s+S)^{2}}\right) k_{1}-D
\end{array}\right]
$$

b). Si $\mu$ se define de acuerdo a la ecuación de Haldane (la cuál es una función no-monótona y también depende únicamente del sustrato):

$$
\mu=\frac{\mu_{m} S}{K_{s}+S+S^{2} / K_{m}}
$$

entonces la matriz $\mathcal{A}$ se define como:

$$
\mathcal{A}(h)=\left[\begin{array}{ll}
\mathcal{A}_{11} & \mathcal{A}_{12} \\
\mathcal{A}_{21} & \mathcal{A}_{22}
\end{array}\right]
$$

donde 


$$
\begin{aligned}
& \mathcal{A}_{11}=\frac{\mu_{m} S}{K s+S+\frac{S^{2}}{K m}}-D \\
& \mathcal{A}_{12}=\frac{\mu_{m} X}{K s+S+\frac{S^{2}}{K m}}-\frac{\mu_{m} S X\left(1+2 \frac{S}{K m}\right)}{\left(K s+S+\frac{S^{2}}{K m}\right)^{2}} \\
& \mathcal{A}_{21}=-\frac{\mu_{m} S}{K s+S+\frac{S^{2}}{K m}} k_{1} \\
& \mathcal{A}_{22}=-\frac{\mu_{m} X}{K s+S+\frac{S^{2}}{K m}}-\frac{\mu_{m} S X\left(1+2 \frac{S}{K m}\right)}{\left(K s+S+\frac{S^{2}}{K m}\right)^{2}} k_{1}-D
\end{aligned}
$$

Ejemplo 3.2 Sea el sistema definido por las siguientes ecuaciones:

$$
\begin{aligned}
\dot{X} & =\varphi_{1}-D X \\
\dot{S} & =-k_{1} \varphi_{1}-k_{2} \varphi_{2}+D\left(S_{i}-S\right) \\
\dot{P} & =\varphi_{2}-D P
\end{aligned}
$$

donde $m=2$ y $n=3$ y la matriz $\mathcal{A}$ se define ahora como:

$$
\begin{aligned}
& \mathcal{A}(h)=h_{11} K \epsilon_{2}(1) \epsilon_{3}(1)^{T}+h_{12} K \epsilon_{2}(1) \epsilon_{3}(2)^{T}+h_{13} K \epsilon_{2}(1) \epsilon_{3}(3)^{T}+ \\
& +h_{21} K \epsilon_{2}(2) \epsilon_{3}(1)^{T}+h_{22} K \epsilon_{2}(2) \epsilon_{3}(2)^{T}+h_{23} K \epsilon_{2}(2) \epsilon_{3}(3)-D I_{3}
\end{aligned}
$$

donde las bases canónica del espacio vectorial son:

$$
\begin{gathered}
\epsilon_{2}(1)=[1 ; 0] \quad \epsilon_{2}(2)=[0 ; 1 \\
\epsilon_{3}(1)=[1 ; \quad 0 ; 0] \epsilon_{3}(2)=\left[\begin{array}{lll}
0 ; & 1 ; & 0
\end{array}\right] \epsilon_{3}(3)=\left[\begin{array}{lll}
1 ; & 0 ; & 1
\end{array}\right]
\end{gathered}
$$

y las Jacobianas de las tasas de reacción se definen como:

$$
\begin{aligned}
& h_{1 j}=\frac{\partial \varphi_{1}}{\partial \xi_{j}} \Rightarrow h_{11}=\frac{\partial \varphi_{1}}{\partial X}, h_{12}=\frac{\partial \varphi_{1}}{\partial S}, h_{13}=\frac{\partial \varphi_{1}}{\partial P} \\
& h_{2 j}=\frac{\partial \varphi_{2}}{\partial \xi_{j}} \Rightarrow h_{21}=\frac{\partial \varphi_{2}}{\partial X}, h_{22}=\frac{\partial \varphi_{2}}{\partial S}, h_{23}=\frac{\partial \varphi_{2}}{\partial P}
\end{aligned}
$$

Así la matriz $\mathcal{A}$ se define para este caso como:

$$
\mathcal{A}=\left[\begin{array}{ccc}
\frac{\partial \varphi_{1}}{\partial X}-D & \frac{\partial \varphi_{1}}{\partial S} & \frac{\partial \varphi_{1}}{\partial P} \\
-k_{1} \frac{\partial \varphi_{1}}{\partial X}-\frac{\partial \varphi_{2}}{\partial X} k_{2}-\frac{\partial \varphi_{1}}{\partial S} k_{1}-\frac{\partial \varphi_{2}}{\partial S} k_{2}-D & -\frac{\partial \varphi_{1}}{\partial P} k_{1}-\frac{\partial \varphi_{2}}{\partial P} k_{2} \\
\frac{\partial \varphi_{2}}{\partial X} & \frac{\partial \varphi_{2}}{\partial S} & \frac{\partial \varphi_{2}}{\partial P}-D
\end{array}\right]
$$

Los vértices del politopo para este ejemplo están definido mediante:

$$
\begin{array}{cl}
\mathcal{A}(\alpha)=\mathcal{A}\left(\alpha_{11}, \alpha_{12}, \alpha_{13}, \alpha_{21}, \alpha_{22}, \alpha_{23}\right) & \\
\alpha_{11} \in \underline{h}_{11}, \bar{h}_{11} & \alpha_{21} \in \underline{h}_{21}, \bar{h}_{21} \\
\alpha_{12} \in \underline{h}_{12}, \bar{h}_{12} & \alpha_{22} \in \underline{h}_{22}, \bar{h}_{22} \\
\alpha_{13} \in \underline{h}_{13}, \bar{h}_{13} & \alpha_{23} \in \underline{h}_{23}, \bar{h}_{23}
\end{array}
$$

Siendo entonces $2^{m n}$ vértices que definen el politopo dentro de los cuales el LMI será factible para definir la matriz de ganancia del observador que satisface dichas condiciones. 


\subsection{Discusión}

En este capítulo se ha presentado el desarrollo de un observador Luenberger cuya matriz de ganancia se determina resolviendo la desigualdad resultante al realizar el análisis de estabilidad mediante Lyapunov empleando LMI.

Esta desigualdad se define a partir de la dinámica del error linealizada cuya matriz característica $\mathcal{A}$ es definida empleando el Teorema del Valor Medio Diferencial. Con esto se cubre el planteamiento del segundo objetivo particular de esta tesis.

Este desarrollo tiene como principales características el considerar las incertidumbres en el modelo, el cuál se considera perfecto, definiendo para ello el intervalo de operación de los estados del mismo, permitiendo con ello definir una ganancia que es válida dentro de los vértices del politopo definido.

Aún cuando la definición de esta matriz depende del modelo matemático, el considerar la incertidumbre en el modelo permite contar con un margen de precisión que le permite mejorar su velocidad de convergencia y su estabilidad en base a la prueba realizada mediante Lyapunov.

Dado que en esencia es un observador Luenberger, podemos extender el concepto a un observador híbrido tipo Luenberger-Asintótico, dado que la sintonización de este se realiza cuando el parámetro que permite la conmutación entre un tipo de observador y otro se ajusta para que el esquema híbrido se comporte como un Luenberger simple.

En el siguiente capítulo se muestra este desarrollo y su aplicación en un observador multifrecuencial, en el cuál el observador asintótico permite converger la estimación hacia el valor real y corregir esta cuando se disponga de la medida realizada fuera de línea. 



\section{Observador multifrecuencial empleando LMI}

A cada paso creamos nuestro propio universo Winston Churchill

\subsection{Introducción}

Como se ha mencionado anteriormente, los bioprocesos son sistemas lentos y no siempre se tienen disponibles en línea todos los parámetros que los definen. El valor de algunos de estos parámetros importantes se conocen su valor mediante análisis realizados fuera de línea y en forma esporádica.

El concepto "multifrecuencial" es empleado aquí indicando que se cuenta con medidas "periódicas frecuentes" provenientes de los sensores disponibles y medidas "esporádicas", cuando el valor de la variable se dispone mediante la medición realizada fuera de línea, en un laboratorio.

En este capítulo se muestra el desarrollo de un observador híbrido resuelto mediante LMI, que se extiende al concepto de multifrecuencial considerando que se cuenta con medidas "periódicas frecuentes".

Estas medidas "frecuentes" están compuesta de las mediciones en línea proporcionadas por los sensores disponibles y, en ciertos instantes de tiempo, se disponen de todas las medidas requeridas. Estas están compuestas por las mediciones en línea y las mediciones fuera de línea disponibles en este instante del tiempo. La estimación es corregida de acuerdo al error presente.

\subsection{Antecedentes}

Uno de los problemas asociados con la monitorización y control de bioprocesos es la ausencia de medidas frecuentes (en línea) de algunas variables esenciales. En estos casos, la estimación continua de estas variables puede lograrse de las mediciones disponibles (frecuentes) y/o fuera de línea (esporádicas), usando un modelo de proceso bajo consideración así como algoritmos de estimación como los mencionados en el capítulo 2. 
La estimación de variables multifrecuenciales en la cuál se empleen las mediciones esporádicas disponibles en la estimación de estados y parámetros permite mejorar la exactitud de la estimación resultante. Los inconvenientes de emplear un filtro Kalman extendido y los recientes avances en el diseño de estimadores de estado no lineales, motivan el uso de estimadores de estados no lineales multifrecuenciales que pueden manejar las no linealidades del proceso y las mediciones multifrecuenciales, [Lubenova et ${ }^{\sim}$ al., 2003].

Varias técnicas de estimación consideran los modelos no lineales de los bioprocesos. Usualmente, los observadores de estados son clasificados como exponenciales o asintóticos de acuerdo a Bastin and Dochain [1990]:

- Los observadores exponenciales tienen una tasa ajustable de convergencia definida por los parámetros de sintonización, sin embargo su eficiencia depende del conocimiento del modelo (Filtro Kalman, Luenberger, Alta ganancia, etc.)

- Los observadores asintóticos no requieren del conocimiento exacto de la cinética del modelo, sin embargo su tasa de convergencia depende de las condiciones experimentales, lo cuál conlleva a convergencias lentas con tasas de disolución bajas.

El observador híbrido es una combinación de un observador exponencial con un observador asintótico, empleando como parámetro controlador entre ambos el error en la medida o el error en el modelado, como se describe en la sección 2.5.3.

El cálculo de la ganancia del observador realizado mediante el procedimiento LMI, se puede emplear para la definición de la ganancia de un observador híbrido Luenberger extendido y Asintótico, si las ganancias cumplen con las condiciones de estabilidad de un observador Luenberger:

$$
\begin{aligned}
|A(\hat{\xi})-\gamma L| & \leq N_{1} & & \forall \hat{\xi} \\
\left|\frac{d}{d t}\{A(\hat{\xi})-\gamma L\}\right| & \leq N_{2} & & \forall \hat{\xi} \\
\mathbb{R e}\left\{\lambda_{i}[A(\hat{\xi})-\gamma L]\right\} & <0 & & \forall \hat{\xi} \quad i=1 . . n
\end{aligned}
$$

En Hulhoven et $\sim a l . ~[2006]$ se recomienda que la sintonización del observador híbrido se realice primero en el caso de cuando el observador se define como un observador Luenberger extendido, y verificar que las condiciones de estabilidad se cumplan para el resto de las posibles combinaciones.

Empleando la solución de un LMI que será válido en el espacio definido por el politopo, las ganancias definidas tendrán raíces negativas para que sea factible, cumpliendo con las condiciones de estabilidad. Así la metodología expuesta puede extenderse y aplicarse para el diseño de un observador híbrido Luenberger extendido y Asintótico. 


\subsection{Desarrollo del Observador híbrido}

En la sección 2.5.3 se presentaron los aspectos generales de un observador híbrido, en esta sección se presenta con mas detalle la propuesta del observador híbrido compuesto de un observador Luenberger extendido y un observador asintótico.

Así, partiendo de la separación de los estados definidos en el observador asintótico y con la variable de estado auxiliar $Z(t)=A_{1} \xi_{1}+A_{2} \xi_{2}$ se obtiene un nuevo esquema para el modelo dinámico general y el observador Luenberger de este modelo :

$$
\left\{\begin{array}{l}
\dot{\hat{\xi}}_{1}=K_{1} \varphi(\hat{\xi})-D \hat{\xi_{1}}+u_{1}+\gamma_{1}(\hat{\xi})\left(y-\hat{\xi}_{1}\right) \\
\dot{\hat{Z}}=-D \hat{Z}+A_{1} u_{1}+A_{2} u_{2}+\gamma_{z}(\hat{\xi})\left(y-\hat{\xi}_{1}\right) \\
\hat{\xi}_{2}=A_{2}^{+}\left(\hat{Z}-A_{1} \hat{\xi}_{1}\right)
\end{array}\right.
$$

Dado que el principal objetivo del observador híbrido es aprovechar las ventajas individuales del observador Luenberger y del observador asintótico, se requiere definir un parámetro que le permita realizar la transición entre uno y otro. Hulhoven et ${ }^{\sim}$ al. [2006] emplea para ello considerar la calidad del modelo dinámico:

$$
\xi_{1} \rightarrow \delta \xi_{1}+(1-\delta) y
$$

donde el parámetro $\delta$ se define como:

$$
\delta=\left(e^{-\frac{\left(y-\hat{\xi_{1}}\right)^{2}}{\sigma^{2}}}-1\right)\left(1-e^{-\frac{t}{\tau}}\right)+1
$$

donde $\sigma^{2}$ corresponde a la confiabilidad de conocimiento del modelo cinético. Así, $\delta$ se desarrolla entre un observador Luenberger extendido y un asintótico, a lo largo del intervalo de $[0,1]$ de acuerdo al error en la estimación de $\xi_{1}$. Entonces, el observador híbrido se redefine como:

$$
\left\{\begin{array}{l}
\dot{\hat{\xi}}_{1}=K_{1} \varphi(\hat{\xi})-D \hat{\xi}_{1}+u_{1}+\gamma_{1}(\hat{\xi})\left(y-\hat{\xi}_{1}\right) \\
\dot{\hat{Z}}=-D \hat{Z}+A_{1} u_{1}+A_{2} u_{2}+\gamma_{z}(\hat{\xi}) \delta\left(y-\hat{\xi}_{1}\right) \\
\hat{\xi}_{2}=A_{2}^{+}\left(\hat{Z}-A_{1}\left(\delta \hat{\xi}_{1}+(1-\delta) y\right)\right)
\end{array}\right.
$$

Así si $\delta \equiv 1$, corresponderá a un observador Luenberger extendido, por el contrario, si $\delta \equiv 0$ corresponderá a un observador asintótico.

Para el análisis de convergencia y sintonización del observador, consideremos que $\delta=1$ tal que el observador híbrido se comporta como un observador Luenberger extendido, ecuación (4.1), cuya dinámica del error $\dot{e}=\left[\begin{array}{ll}\dot{e}_{1} & \dot{e}_{z}\end{array}\right]^{T}$ está definida mediante:

$$
\begin{aligned}
& \dot{e}_{1}=K_{1}\{\varphi(\xi)-\varphi(\hat{\xi})\}-D e_{1}-\gamma_{1} e_{1} \\
& \dot{e}_{z}=-D e_{z}-\gamma_{z} e_{1}
\end{aligned}
$$


donde $e_{1}=\xi_{1}-\hat{\xi}_{1}$ y $e_{z}=Z-\hat{Z}$.

Para la solución de $\varphi(\xi)-\varphi(\hat{\xi})$ emplearemos el Teorema del Valor Medio Diferencial (DMVT).

\subsubsection{Resolución mediante DMVT}

Dado que los estados del nuevo modelo se separaron y redefinieron, $\xi=$ $\left[\begin{array}{ll}\xi_{1} & Z\end{array}\right]^{T}$, entonces el error existente entre las tasas de reacción se define ahora como $\varphi\left(\xi_{1}, Z\right)-\varphi\left(\hat{\xi}_{1}, \hat{Z}\right)$. Siguiendo entonces la estrategia definida en 3.5 , aplicamos el concepto de DMVT para su solución.

Sea :

- $\xi_{1}^{*} \in C o\left(\xi_{1}, \hat{\xi}_{1}\right) \quad \mid \quad\left\{\xi_{1}^{*} \neq \xi_{1}, \xi_{1}^{*} \neq \hat{\xi}_{1}\right\}$

- $Z^{*} \in C o(Z, \hat{Z}) \mid\left\{Z^{*} \neq Z, Z^{*} \neq \hat{Z}\right\}$

entonces definimos:

$$
\begin{array}{r}
\varphi\left(\xi_{1}, Z\right)-\varphi\left(\hat{\xi}_{1}, \hat{Z}\right)=\frac{\partial \varphi\left(\xi_{1}^{*}, Z^{*}\right)}{\partial \xi_{1}}\left(\xi_{1}-\hat{\xi}_{1}\right)+\frac{\partial \varphi\left(\xi_{1}^{*}, Z^{*}\right)}{\partial Z}(Z-\hat{Z}) \\
=\left(\sum_{i, j=1}^{m, q} \epsilon_{m}(i) \epsilon_{q}^{T}(j) \frac{\partial \varphi_{i}\left(\xi_{1}^{*}, Z^{*}\right)}{\partial \xi_{1, j}}\right) e_{1}+\left(\sum_{i, j=1}^{m, n-q} \epsilon_{m}(i) \epsilon_{n-q}^{T}(j) \frac{\partial \varphi_{i}\left(\xi_{1}^{*}, Z^{*}\right)}{\partial Z_{j}}\right) e_{z}
\end{array}
$$

Si $h_{1(i, j)}=\frac{\partial \varphi_{i}\left(\xi_{1}^{*}, Z^{*}\right)}{\partial \xi_{1, j}}$ y $h_{z(i, j)}=\frac{\partial \varphi_{i}\left(\xi_{1}^{*}, Z^{*}\right)}{\partial Z_{j}}$, y se define:

$$
\begin{aligned}
& \mathcal{H}_{1}=\sum_{i, j=1}^{m, q} \epsilon_{m}(i) \epsilon_{q}^{T}(j) h_{1(i, j)} \\
& \mathcal{H}_{z}=\sum_{i, j=1}^{m, n-q} \epsilon_{m}(i) \epsilon_{n-q}^{T}(j) h_{z(i, j)}
\end{aligned}
$$

entonces $K_{1}\{\varphi(\xi)-\varphi(\hat{\xi})\}$ queda definido por:

$$
K_{1}\{\varphi(\xi)-\varphi(\hat{\xi})\}=K_{1} \mathcal{H}_{1}\left(h_{1}\right) e_{1}+K_{1} \mathcal{H}_{z}\left(h_{z}\right) e_{z}
$$

Dado que podemos definir los intervalos entre los cuales se encuentra las derivadas parciales de las tasas de reacción, definimos ahora $\alpha_{1}=\left(\alpha_{11}, \ldots, \alpha_{1 q}\right) \in$ $\left\{\bar{h}_{1(i, j)}, \underline{h}_{1(i, j)}\right\}$ y $\alpha_{z}=\left(\alpha_{z 1}, \ldots, \alpha_{z(n-q)}\right) \in\left\{\bar{h}_{z(i, j)}, \underline{h}_{z(i, j)}\right\}$, teniendo entonces que la ecuación anterior considerando estos intervalos será:

$$
K_{1}\{\varphi(\xi)-\varphi(\hat{\xi})\}=K_{1} \mathcal{H}_{1}\left(\alpha_{1}\right) e_{1}+K_{1} \mathcal{H}_{z}\left(\alpha_{z}\right) e_{z}
$$

La dinámica del error para este modelo entonces se expresa como:

$$
\left\{\begin{array}{l}
\dot{e}_{1}=K_{1} \mathcal{H}_{1}\left(\alpha_{1}\right) e_{1}+K_{1} \mathcal{H}_{z}\left(\alpha_{z}\right) e_{z}-D e_{1}-\gamma_{1} e_{1} \\
\dot{e}_{z}=-D e_{z}-\gamma_{z} e_{1}
\end{array}\right.
$$

Expresando esta ecuación en forma matricial:

$$
\dot{e}=\left(\left[\begin{array}{cc}
K_{1} \mathcal{H}_{1}\left(\alpha_{1}\right)-D I_{q} & K_{1} \mathcal{H}_{z}\left(\alpha_{z}\right) \\
0 & -D I_{n-q}
\end{array}\right]-\left[\begin{array}{ll}
\gamma_{1} & 0 \\
\gamma_{z} & 0
\end{array}\right]\right)\left[\begin{array}{l}
e_{1} \\
e_{z}
\end{array}\right]
$$




\subsection{Observador híbrido multifrecuencial}

Consideremos ahora que tenemos un comportamiento multifrecuencial, en el cuál se cuenta con medidas periódicas frecuentes y medidas esporádicas. Las medidas periódicas frecuentes o rápidas son "instantáneas" en el sentido de que la medición es realizada y está disponible en el mismo instante de muestreo. Las medidas esporádicas o lentas son tomadas en un instante de muestreo pero están disponibles hasta algunos instantes de muestreo posteriores debido al tiempo de retardo asociado con el análisis de la muestra.

El esquema multifrecuencial del observador híbrido se obtiene si consideramos que en cada muestreo existen dos alternativas para la estimación, de acuerdo a que medida (en línea y/o fuera de línea) esté disponibles, [Tatiraju et ${ }^{\sim}$ al., 1999a]. Entonces definimos dos observadores de acuerdo a la disponibilidad de las medidas:

1. Observador híbrido durante las medidas periódicas frecuentes. Este observador estará en operación durante los instantes de muestreo en los cuales no se cuenta con el valor de la variable medida fuera de línea $\left(\xi_{2}=0\right)$

2. Observador híbrido durante las medidas esporádicas. Cuando se dispone del valor de la variable medida fuera de línea, este observador entra en operación y entonces se realiza el ajuste del modelo en base al error existente.

\subsubsection{Observador híbrido durante las medidas periódicas frecuentes}

Consideremos entonces que la medición de un grupo de estados es realizada $\left(y=\xi_{1}\right)$ y está disponible en el mismo instante de muestreo y no se cuenta con el valor de la variable medida fuera de línea, entonces el observador queda definido por:

$$
\left\{\begin{array}{l}
\dot{\hat{\xi}}=K_{1} \varphi\left(\hat{\xi}_{1}, \hat{Z}\right)-D \hat{\xi} 1+u_{1}+\gamma_{1 p}(\hat{\xi})\left(\xi_{1}-\hat{\xi}_{1}\right) \\
\dot{\hat{Z}}=-D \hat{Z}+A_{1} u_{1}+A_{2} u_{2}+\gamma_{z p}(\hat{\xi})\left(\xi_{1}-\hat{\xi}_{1}\right)
\end{array}\right.
$$

donde $\varphi \in \mathbb{R}^{m}, \xi_{1} \in \mathbb{R}^{q}$ y $Z \in \mathbb{R}^{n-q}$. El estado estimado se determina mediante $\hat{\xi}_{2}=A_{2}^{+}\left(\hat{Z}-A_{1}\left(\delta \hat{\xi}_{1}+(1-\delta) \xi_{1}\right)\right)$

La dinámica del error para este modelo es:

$$
\begin{aligned}
& \dot{e}_{1}=K_{1}\left\{\varphi\left(\xi_{1}, Z\right)-\varphi\left(\hat{\xi}_{1}, \hat{Z}\right)\right\}-D e_{1}-\gamma_{1 p} e_{1} \\
& \dot{e}_{z}=-D e_{z}-\gamma_{z p} e_{1}
\end{aligned}
$$

donde $e_{1}=\xi_{1}-\hat{\xi}_{1}$ y $e_{z}=Z-\hat{Z}$. Resolviendo mediante DMVT la dinámica del error, entonces se expresa como:

$$
\left\{\begin{array}{l}
\dot{e}_{1}=K_{1} \mathcal{H}_{1}\left(\alpha_{1}\right) e_{1}+K_{1} \mathcal{H}_{z}\left(\alpha_{z}\right) e_{z}-D e_{1}-\gamma_{1 p} e_{1} \\
\dot{e}_{z}=-D e_{z}-\gamma_{z p} e_{1}
\end{array}\right.
$$


Expresando esta ecuación en forma matricial:

$$
\dot{e}=\left(\left[\begin{array}{cc}
K_{1} \mathcal{H}_{1}\left(\alpha_{1}\right)-D I_{q} & K_{1} \mathcal{H}_{z}\left(\alpha_{z}\right) \\
0 & -D I_{n-q}
\end{array}\right]-\left[\begin{array}{ll}
\gamma_{1 p} & 0 \\
\gamma_{z p} & 0
\end{array}\right]\right)\left[\begin{array}{l}
e_{1} \\
e_{z}
\end{array}\right]
$$

si definimos que:

$$
\mathcal{A}\left(\alpha_{1}, \alpha_{z}\right)=\left[\begin{array}{cc}
K_{1} \mathcal{H}_{1}\left(\alpha_{1}\right)-D I_{q} & K_{1} \mathcal{H}_{z}\left(\alpha_{z}\right) \\
0 & -D I_{n-q}
\end{array}\right]
$$

entonces la dinámica del error la podemos reescribir de la forma:

$$
\dot{e}=\left(\mathcal{A}\left(\alpha_{1}, \alpha_{z}\right)-\mathcal{G}_{c}\right) e
$$

donde $\mathcal{G}_{c}$ es la matriz de ganancias para el caso de medidas periódicas frecuentes, la cuál solo tiene valor diferente de cero en la columna uno, correspondiente al error en la estimación de los estados medidos.

\subsubsection{Observador híbrido durante las medidas esporádicas}

Para esta etapa, la medidas esporádicas $\left(\xi_{2}\right)$ están disponible algunos instantes de muestreo posteriores, este retardo está asociado con el análisis de la muestra. Dicho retardo se considera asíncrono y para efectos del trabajo, durante ese periodo, opera el observador híbrido descrito anteriormente.

Cuando se dispone del valor real de $\xi_{2}$, el cuál proviene de una medición fuera de línea, se realiza el ajuste del modelo en base al error existente entre este valor y el valor estimado en el tiempo anterior.

De manera que en este instante se cuenta con todas las lecturas ( $\xi_{1}$ y $\left.\xi_{2}\right)$ y se determina el error respecto a los valores estimados. Así el observador híbrido se define:

$$
\left\{\begin{array}{l}
\dot{\hat{\xi}}_{1}=K_{1} \varphi\left(\hat{\xi}_{1}, \hat{Z}\right)-D \hat{\xi}_{1}+u_{1}+\gamma_{1 e}(\hat{\xi})\left(\xi_{1}-\hat{\xi}_{1}\right) \\
\left.\dot{\hat{Z}}=-D \hat{Z}+A_{1} u_{1}+A_{2} u_{2}+\gamma_{z e}(\hat{\xi})(Z-\hat{Z})\right)
\end{array}\right.
$$

donde $\varphi \in \mathbb{R}^{m}, \xi_{1} \in \mathbb{R}^{q}$ y $Z \in \mathbb{R}^{n-q}, Z(t)=A_{1} \xi_{1}+A_{2} \xi_{2}, \hat{Z}(t)=A_{1} \hat{\xi}_{1}+A_{2} \hat{\xi}_{2}$. Se estima el nuevo valor de $\xi_{2}$ mediante $\hat{\xi}_{2}=A_{2}^{+}\left(\hat{Z}-A_{1}\left(\delta \hat{\xi}_{1}+(1-\delta) \xi_{1}\right)\right)$. Sea $e_{1}=\xi_{1}-\hat{\xi}_{1}$ y $e_{z}=Z-\hat{Z}$ los errores asociados a cada una de las particiones del estado, entonces la dinámica del error para este modelo se define como:

$$
\begin{aligned}
& \dot{e}_{1}=K_{1}\left\{\varphi\left(\xi_{1}, Z\right)-\varphi\left(\hat{\xi}_{1}, \hat{Z}\right)\right\}-D e_{1}-\gamma_{1 e} e_{1} \\
& \dot{e}_{z}=-D e_{z}-\gamma_{z e} e_{z}
\end{aligned}
$$

Resolviendo mediante DMVT la dinámica del error se expresa como:

$$
\left\{\begin{array}{l}
\dot{e}_{1}=K_{1} \mathcal{H}_{1}\left(\alpha_{1}\right) e_{1}+K_{1} \mathcal{H}_{z}\left(\alpha_{z}\right) e_{z}-D e_{1}-\gamma_{1 e} e_{1} \\
\dot{e}_{z}=-D e_{z}-\gamma_{z e} e_{z}
\end{array}\right.
$$

Expresando esta ecuación en forma matricial: 


$$
\dot{e}=\left(\left[\begin{array}{cc}
K_{1} \mathcal{H}_{1}\left(\alpha_{1}\right)-D I_{q} & K_{1} \mathcal{H}_{z}\left(\alpha_{z}\right) \\
0 & -D I_{n-q}
\end{array}\right]-\left[\begin{array}{cc}
\gamma_{1 e} & 0 \\
0 & \gamma_{z e}
\end{array}\right]\right)\left[\begin{array}{l}
e_{1} \\
e_{z}
\end{array}\right]
$$

la cuál es de la forma:

$$
\dot{e}=\left(\mathcal{A}\left(\alpha_{1}, \alpha_{z}\right)-\mathcal{G}_{e}\right) e
$$

donde $\mathcal{G}_{e}$ es la matriz de ganancias cuando se tiene el valor medido del estado estimado, cuya diagonal principal está conformada por las ganancias individuales de los errores en la estimación del estado medido en línea y el valor actual del estado que se está realizando su medición fuera de línea, para la corrección de la desviación que existiera.

\subsubsection{Análisis de estabilidad}

Considerando la función cuadrática de Lyapunov:

$$
\begin{aligned}
& V(e)=e^{T} P e \\
& \dot{V}(e)=\dot{e}^{T} P e+e^{T} P \dot{e}
\end{aligned}
$$

Sea $P_{1}=P_{1}^{T}>0, P_{2}=P_{2}^{T}>0, R_{1}=\mathcal{G}_{c}^{T} P$ y $R_{2}=\mathcal{G}_{e}^{T} P$, el error de observación $e(t)$ converge exponencialmente hacia cero si los siguientes LMI son factibles:

Medidas periódicas frecuentes

$$
\left\{\begin{array}{l}
\mathcal{A}^{T}\left(\alpha_{1}, \alpha_{z}\right) P_{1}-R_{1}+P_{1} \mathcal{A}\left(\alpha_{1}, \alpha_{z}\right)-R_{1}^{T}<0 \\
P_{1}>0
\end{array}\right.
$$

Medidas esporádicas

$$
\left\{\begin{array}{l}
\mathcal{A}^{T}\left(\alpha_{1}, \alpha_{z}\right) P_{2}-R_{2}+P_{2} \mathcal{A}\left(\alpha_{1}, \alpha_{z}\right)-R_{2}^{T}<0 \\
P_{2}>0
\end{array}\right.
$$

Cuando los LMI sean factibles, las matrices de ganancia se determinan por $\mathcal{G}_{c}=P_{1}^{-1} R_{1}^{T}$ y $\mathcal{G}_{e}=P_{2}^{-1} R_{2}^{T}$, las cuales serán válidas dentro del politopo cuyos vértices están formados por $\alpha_{1}$ y $\alpha_{z}$

\subsection{Análisis del algoritmo}

Como se observa en las secciones anteriores, la dinámica del error resuelta mediante DMVT está definida mediante la siguiente ecuación:

$$
\dot{e}=\left[\begin{array}{cc}
K_{1} \mathcal{H}_{1}\left(\alpha_{1}\right)-D I_{q} & K_{1} \mathcal{H}_{z}\left(\alpha_{z}\right) \\
0 & -D I_{n-q}
\end{array}\right]\left[\begin{array}{l}
e_{1} \\
e_{z}
\end{array}\right]-\mathcal{G e}
$$

donde la matriz de ganancias $\mathcal{G}$ se define de acuerdo a las medidas disponibles : 
- Medida Continua: $\mathcal{G}_{c}=\left[\begin{array}{cc}\gamma_{1 p} & 0 \\ \gamma_{z p} & 0\end{array}\right]$

- Medida Esporádica: $\mathcal{G}_{e}=\left[\begin{array}{cc}\gamma_{1 e} & 0 \\ 0 & \gamma_{z e}\end{array}\right]$

Así, el algoritmo del observador multifrecuencial puede describirse de la siguiente manera:

En el tiempo $t=0$, el algoritmo se inicializa con las condiciones iniciales, las cuales son los valores actuales (medidos), $\xi_{1}$. El conjunto de las ecuaciones diferenciales ordinarias del estimador son integradas numéricamente con un paso de integración $\tau$ (periodo de muestreo de la medida). Esta integración numérica es repetida cuando el siguiente valor de la medida se tenga disponible. En cada instante de tiempo $t_{j}$ la estimación disponible (o calculada) de la variable de estado del proceso correspondiente al instante de tiempo previo $\left(t_{j-1}\right)$ es usado como condiciones iniciales y el valor actual de la medida y la medida esporádica más actualizada de la variable de estado son alimentadas al estimador para calcular la estimación de tiempo presente de las variables de estado.

Mientras no se cuente con las medidas fuera de línea, el observador se comporta como un observador híbrido Luenberger-Asintótico cuyo parámetro $\delta$ dependerá del error en la medida.

Las medidas resultantes de los análisis fuera de línea de una muestra tomada en un tiempo $t_{i}$ están disponibles en diferentes instantes de tiempo y al contar con dicho valor se realiza la corrección en la estimación del estado $\xi_{2}$.

\subsection{Discusión}

En este capítulo se presentó el desarrollo de un observador híbrido Luenberger-Asintótico cuya matriz de ganancia se determinó ajustándolo como solo Luenberger y resolviéndolo mediante el procedimiento de LMI que se describió en el capítulo anterior.

Este concepto de observador híbrido se aplica a un sistema multifrecuencial definido por las medidas disponibles en línea y las medidas realizadas fuera de línea y con retardo, teniendo entonces el desarrollo de un observador híbrido multifrecuencial. Así, el tercer objetivo particular planteado para la tesis es cubierto mediante este desarrollo.

En el siguiente capítulo se aplicarán los tres conceptos vertidos hasta este punto: observador para bioprocesos empleando LMI, observador híbrido resuelto mediante LMI y observador híbrido multifrecuencial. Para ello se empleará la simulación de modelos matemáticos y se analizará su desempeño comparándolo con un observador asintótico clásico. 


\section{Casos de Estudio}

Me lo contaron y lo olvidé, lo vi y lo entendí, lo hice y lo aprendí

Confucio

\subsection{Introducción}

En el presente capítulo se estudian dos casos para la aplicación de los desarrollos anteriores, descritos en dos grandes secciones: a). para un sistema productor metabólico se realiza la estimación mediante la aplicación del observador Luenberger extendido, cuyas ganancias se determinan mediante el procedimiento empleando LMI, y un observador híbrido resuelto mediante LMI; b). un sistema multifrecuencial, en donde se emplea un observador híbrido multifrecuencial resuelto mediante LMI para mostrar la extensión de la propuesta a este tipo de sistemas.

\subsection{Modelos de fermentación}

Los modelos matemáticos describen el comportamiento de un bioproceso y son la base fundamental para el desarrollo de los estimadores de estado y/o parámetros. Para los sistemas de bioprocesos, estos se clasifican en, [Shimizu, 1996]:

Sistema 1. Sistema productor de biomasa que se representan mediante ecuaciones de la forma:

$$
\left[\begin{array}{c}
\dot{X} \\
\dot{S}
\end{array}\right]=\left[\begin{array}{c}
1 \\
-k_{s}
\end{array}\right] \varphi-\left[\begin{array}{c}
X \\
S
\end{array}\right] D+\left[\begin{array}{c}
0 \\
D S_{i n}
\end{array}\right]
$$

Sistema 2. Sistema productor metabólico de la forma:

$$
\left[\begin{array}{c}
\dot{X} \\
\dot{S} \\
\dot{P}
\end{array}\right]=\left[\begin{array}{cc}
1 & 0 \\
-k_{s} & k_{p} \\
0 & 1
\end{array}\right]\left[\begin{array}{l}
\varphi_{1} \\
\varphi_{2}
\end{array}\right]-\left[\begin{array}{c}
X \\
S \\
P
\end{array}\right] D+\left[\begin{array}{c}
0 \\
D S_{\text {in }} \\
0
\end{array}\right]
$$


Sistema 3. Sistemas de biorreactores múltiples, cuyo modelo basado en experimentos tipo lote es:

$$
\left[\begin{array}{c}
\dot{X}_{v} \\
\dot{X}_{n v} \\
\dot{X}_{d} \\
\dot{P}
\end{array}\right]=\left[\begin{array}{cccc}
X_{v}-X_{v} & 0 & 0 \\
0 & X_{v} & -X_{n v} & 0 \\
0 & 0 & X_{n v} & 0 \\
0 & 0 & \left(X_{v}+X_{n v}\right)
\end{array}\right]\left[\begin{array}{c}
\mu(P) \\
\kappa(P) \\
\gamma \\
\pi(P)
\end{array}\right]
$$

Sistema 4. Sistemas de fermentación extractiva (por ejemplo fermentación extractiva de acetona-butanol usando alcohol oléico).

$$
\left[\begin{array}{c}
\dot{X} \\
\dot{S} \\
P_{i} V+P_{i}^{*} V^{*}
\end{array}\right]=\left[\begin{array}{c}
X \\
-\frac{X}{Y} \\
\frac{X V}{Y_{i}}
\end{array}\right] \mu\left(S, P_{1}, P_{2}, \ldots, P_{n}\right)
$$

$(i=1,2, \ldots, n)$

Sistema 5. Sistemas de reciclaje de células con filtración (por ejemplo acidos carboxilicos como la lactosa).

$$
\left[\begin{array}{c}
\dot{X} \\
\dot{P} \\
\dot{S}
\end{array}\right]=\left[\begin{array}{ccc}
X & 0 & 0 \\
0 & X & 0 \\
0 & 0 & -X
\end{array}\right]\left[\begin{array}{l}
\mu(S, P) \\
\pi(S, P) \\
\sigma(S, P)
\end{array}\right]+\left[\begin{array}{c}
0 \\
-D P \\
D\left(S_{\text {in }}-S\right)
\end{array}\right]
$$

Todos los casos se considerarán de fermentaciones continuas o de semilote. En el desarrollo de este capítulo se empleará un sistema tipo 1 y 2, es decir producto de biomasa y metabólico.

Se aplicará el desarrollo del observador para bioprocesos empleando LMI, y una extensión de uso a un observador híbrido que es la base del desarrollo del observador multifrecuencial empleando LMI. En ambos casos se comparan los resultados con los obtenidos empleando un observador asintótico clásico de lazo abierto y un filtro Kalman extendido.

\subsubsection{Análisis de resultados}

En el presente capítulo se presentan la simulación de la aplicación de la metodología expuesta anteriormente a modelos de bioprocesos. Los resultados obtenidos se comparan con los resultados que se logran al aplicar la metodología del observador asintótico. El análisis de los resultados se realiza mediante un análisis del error existente entre el valor estimado y el medido. Por definición fundamental, un error es la desviación de las cantidades medidas del valor "real". Los errores experimentales pueden ser de dos tipos [Simanek, 1996]:

Indeterminados. Se encuentran presentes en todas las mediciones experimentales y se refieren a errores que no hay forma de determinar su tamaño o signo en cualquier medida individual. Puede producirse debido a errores humanos, cambios en las condiciones experimentales, variaciones ambientales y a la variabilidad inherente en los instrumentos de medición. 
Determinados o Sistemáticos. "Sistemáticos" significa que cuando la medición de una cantidad es repetida en varias ocasiones, el error tiene el mismo tamaño y signo algebraico en cualquier medición. "Determinados" significa que el tamaño y el signo del error pueden ser definidos. El error determinado puede producirse por una mala calibración del instrumento, uso de valores inadecuados de las constantes en las ecuaciones, uso de unidades inadecuadas, etc.

Si disponemos de dos o más mediciones las cuales requerimos comparar para determinar sus características entre ellas, existen tres formas estándar para la comparación de estas cantidades:

Desviación. Cuando un conjunto de mediciones es realizada de una cantidad física, es útil expresar la diferencia entre cada medición y el promedio del conjunto completo: la desviación de la medida de la media.

Diferencia. Hay situaciones donde es necesario comparar las mediciones o resultados los cuales se asumen están en igualdad de confiabilidad

Discrepancia experimental. Cuando una medición o resultado es comparado con otro el cuál se conoce es más confiable.

Ya que con frecuencia se cuenta con un vector de valores medidos y estimados cuya dimensión dependerá del número de muestra, es de ayuda realizar un resumen estadístico de sus características, siendo las más usadas la media y la desviación estándar.

Media. Representa la suma de los números de la muestra, dividido entre la cantidad total de números, $N$.

$$
\bar{\theta}=\frac{\sum_{i=1}^{N}}{N}
$$

Desviación estándar de la media, DEM. Para un conjunto de $N$ mediciones $\theta$ cuyo valor medio es $\bar{\theta}$, la desviación estándar de la media es:

$$
D E M=\sqrt{\frac{\sum_{i=1}^{N} \theta^{2}-N \bar{\theta}^{2}}{N(N-1)}}
$$

El error existente en una medida y la estimación puede expresarse de dos manera:

Error Absoluto, EA. Las incertidumbres pueden ser expresadas como medidas absolutas: $E A=\hat{\theta}-\theta$

Error relativo, ER. Las incertidumbres pueden ser expresadas como medidas relativas, dadas por la relación entre la incertidumbre de las cantidades y la cantidad en sí, es decir:

$$
E R(\%)=\frac{E A}{\theta} * 100
$$


Sin embargo, si estos valores son muy próximos entre sí, se requerirá indicar cual medida o estimación es precisa. Si por precisión se entiende el promedio del grado de correspondencia entre pares individuales de valores medidos y estimados, entonces para determinar la precisión podemos emplear el error cuadrático medio (ECM), el cuál proporciona la medida de las diferencias en promedio entre los valores medidos y estimados. El error cuadrático medio de un estimador $\hat{\theta}$ se define como, [Hines and Montgomery, 2004]:

$$
\operatorname{ECM}(\hat{\theta})=\sqrt{\sum_{i=1}^{N} \frac{(\hat{\theta}-\theta)^{2}}{N}}
$$

donde $\hat{\theta}$ es el valor estimado, $\theta$ es el valor medido. El ECM nos da la medida de las diferencias en promedio entre los valores estimados y los medidos, siendo un criterio importante para comparar dos estimadores. Sean $\hat{\theta}_{1}$ y $\hat{\theta}_{2}$ dos estimadores del parámetro $\theta, \mathrm{y} \operatorname{ECM}\left(\hat{\theta}_{1}\right)$ y $\operatorname{ECM}\left(\hat{\theta}_{2}\right)$ los errores cuadráticos medios de $\hat{\theta}_{1}$ y $\hat{\theta}_{2}$, entonces la eficiencia relativa de $\hat{\theta}_{2}$ a $\hat{\theta}_{1}$ se define como

$$
E f_{\text {rel }}=\frac{E C M\left(\hat{\theta}_{1}\right)}{\operatorname{ECM}\left(\hat{\theta}_{2}\right)}
$$

Si esta $E f_{\text {rel }}$ es menor que uno, concluiríamos que $\hat{\theta}_{1}$ es un estimador más eficiente de $\theta$ que $\hat{\theta}_{2}$ en el sentido de que tiene un error cuadrático medio más pequeño.

\subsection{Modelo de fermentación - Sistema Productor de Biomasa}

Consideremos un sistema productor de biomasa $(\mathrm{X})$ en un proceso simple de crecimiento microbiano en un sustrato (S). El modelo dinámico en un reactor continuo está definido por Dochain [2003]:

$$
\begin{aligned}
\dot{X} & =\mu X-D X \\
\dot{S} & =-k_{1} \mu X+D S_{i n}-D S
\end{aligned}
$$

donde el orden del sistema es $\mathrm{n}=2, k_{1}$ es el coeficiente de producción, y la tasa de crecimiento específico $\mu$ está definida por la cinética de Monod:

$$
\mu=\frac{\mu_{\max } S}{K_{S}+S}
$$

donde $\mu_{\max }$ y $K_{S}$ representan la tasa máxima de crecimiento y la constante de saturación respectivamente. Las condiciones numéricas para la simulación son las descritas en la siguiente tabla: 


\begin{tabular}{|c|c|c|c|c|c|c|}
\hline$k_{1}$ & $\mu_{\max }$ & $K_{S}$ & $S_{\text {in }}$ & $\mathrm{D}$ & $\mathrm{X}(0)$ & $\mathrm{S}(0)$ \\
\hline 2 & $0.33 h^{-1}$ & $5 \mathrm{~g} / \mathrm{L}$ & $5 \mathrm{~g} / \mathrm{L}$ & $0.05 h^{-1}$ & $1 \mathrm{~g} / \mathrm{L}$ & $0.5 \mathrm{~g} / \mathrm{L}$ \\
\hline
\end{tabular}

Expresando la ecuación que define al bioproceso con el modelo dinámico general, ecuación (2.2):

$$
\begin{aligned}
{\left[\begin{array}{c}
\dot{X} \\
\dot{S}
\end{array}\right] } & =\left[\begin{array}{c}
1 \\
-k_{1}
\end{array}\right][\mu X]-D\left[\begin{array}{c}
X \\
S
\end{array}\right]+\left[\begin{array}{c}
0 \\
D S_{i}
\end{array}\right] \\
y & =L \xi
\end{aligned}
$$

\subsubsection{Análisis de Observabilidad}

La matriz $L$ puede definirse aplicando el análisis de observabilidad:

$$
\begin{aligned}
\mathcal{O} & =\left[\begin{array}{c}
L \\
L A(\xi)
\end{array}\right] \\
A(\xi) & =\left[\begin{array}{c}
1 \\
-k_{1}
\end{array}\right]\left[\begin{array}{ll}
\varphi_{X} & \varphi_{S}
\end{array}\right]-D I_{n} \\
\varphi_{X} & =\frac{\partial \varphi}{\partial X}=\frac{\mu_{m} S}{K s+S} \\
\varphi_{S} & =\frac{\partial \varphi}{\partial S}=\frac{\mu_{m} X}{K s+S}-\frac{\mu_{m} S X}{(K s+S)^{2}}
\end{aligned}
$$

y al análisis de observabilidad local

$$
\operatorname{rank}(\mathcal{O} \mathcal{L})=\operatorname{rank}\left\{d h, d L_{f} h, d L_{g} h\right\}^{T}=2
$$

Si $L=\left[\begin{array}{ll}1 & 0\end{array}\right]$ o $L=\left[\begin{array}{ll}0 & 1\end{array}\right]$ para ambos casos el rango de la matriz $\mathcal{O}$ y $\mathcal{O} \mathcal{L}$ es 2 , indicando con ello que la medida de cualquiera nos proporciona la información necesaria para el desarrollo del observador.

\subsubsection{Observador Luenberger empleando LMI}

En esta sección se muestra la aplicación del desarrollo del observador Luenberger extendido resuelto mediante DMVT y cuyas ganancias son determinadas empleando el procedimiento LMI. Los resultados se comparan con un filtro Kalman extendido y un observador asintótico de lazo abierto. El observador para el proceso de la ecuación (5.26) se define como:

$$
\begin{aligned}
\dot{\hat{\xi}} & =K \varphi(\hat{\xi})-D \hat{\xi}+u+\gamma L\left[\xi_{1}-\hat{\xi_{1}}\right] \\
y & =L \xi
\end{aligned}
$$

donde $L=\left[\begin{array}{ll}0 & 1\end{array}\right]$ y $u=F-Q$. La dinámica del error para este observador queda definida mediante la ecuación (2.16) y resuelta mediante el teorema del valor medio diferencial :

$$
\dot{e}(t)=(\mathcal{A}(h(t))-\gamma L) e(t)
$$


donde la matriz $\mathcal{A}$ se define por:

$$
\mathcal{A}(h(t))=\sum_{i, j=1}^{m, n} h_{i j}(t) K \epsilon_{m}(i) \epsilon_{n}^{T}(j)-D I_{n}
$$

y $m=1, n=2$ y $h_{i j}=h_{1 j}=\frac{\partial \mu(S) X}{\partial \xi_{j}}, j=1, \ldots, n$. La estructura de la matriz $\mathcal{A}(h(t))$ se describe en la sección 3.5

Dado que $S_{\max }=S_{i n}$, entonces los límites de las concentraciones son: $X=\{0,2,5\} g / l, S=\{0,5\} g / l$ Los valores máximos y mínimos de $h_{i}$ son:

$$
\begin{aligned}
& \underline{h}_{1 j}=\left[\begin{array}{l}
0,0065 \\
0,0063 \\
0,1650 \\
0,0413
\end{array}\right] \\
& \bar{h}_{1 j}=
\end{aligned}
$$

El LMI

$$
\left\{\begin{array}{c}
\mathcal{A}(h(t))^{T} P-C^{T} R+P \mathcal{A}(h(t))-R^{T} C<0 \\
P>0
\end{array}\right.
$$

donde $h(t)=\left(\underline{h}_{i}, \bar{h}_{i}\right), R=\gamma^{T} P$ y $P=P^{T}$,es factible de acuerdo al desarrollo empleando Matlab, y su solución proporciona los valores de la matriz de ganancia:

$$
\gamma=\left[\begin{array}{ll}
-1,1182 & 1,2379
\end{array}\right]^{T}
$$

\subsubsection{Observador Kalman Extendido}

El observador extendido para este sistema no lineal se define como:

$$
\begin{aligned}
\dot{\hat{\xi}} & =K \varphi\left(\xi_{1}, \hat{\xi}_{2}\right)-D \hat{\xi}+u+\gamma\left[\xi_{1}-\hat{\xi_{1}}\right] \\
\xi_{1} & =L \xi
\end{aligned}
$$

donde $\varphi\left(\xi_{1}, \hat{\xi}_{2}\right)=\mu X, u=D S_{i}$ y $L=\left[\begin{array}{ll}0 & 1\end{array}\right]$, es decir, consideramos que se mide en línea el sustrato (S) y $\gamma$ se define mediante la ecuación de Ricatti.

$$
\frac{d R}{d t}=-R L^{T} L R+R A(\hat{\xi})^{T}+A(\hat{\xi}) R
$$

donde: $\gamma=R L^{T}$

\subsubsection{Observador Asintótico}

Para el desarrollo del observador asintótico consideramos también que el sustrato es medido en línea, así $\xi_{1}=S$ y $\xi_{2}=X$ y siguiendo la metodología planteada en Bastin and Dochain [1990] obtenemos la siguiente ecuación: 


$$
\begin{gathered}
\dot{\hat{Z}}=-D \hat{Z}+A_{0} D \xi_{i 1} \\
\hat{\xi}_{2}=\hat{Z}-A_{0} \xi_{1} \\
\text { donde } A_{0}=-K_{2} K_{1}^{-1} \text { y } \xi_{i}=\left[\xi_{i 1}, \xi_{i 2}\right]^{T}=\left[S_{i}, 0\right]^{T}
\end{gathered}
$$

\subsubsection{Resultados}

La aplicación de cada uno de los esquemas de observadores mostrados anteriormente se realiza sobre el modelo simulado en Matlab. La figura 5.1 muestra el comportamiento del error en la estimación de la biomasa (X) a lo largo de 80 hrs de simulación. Como se observa los observadores inician

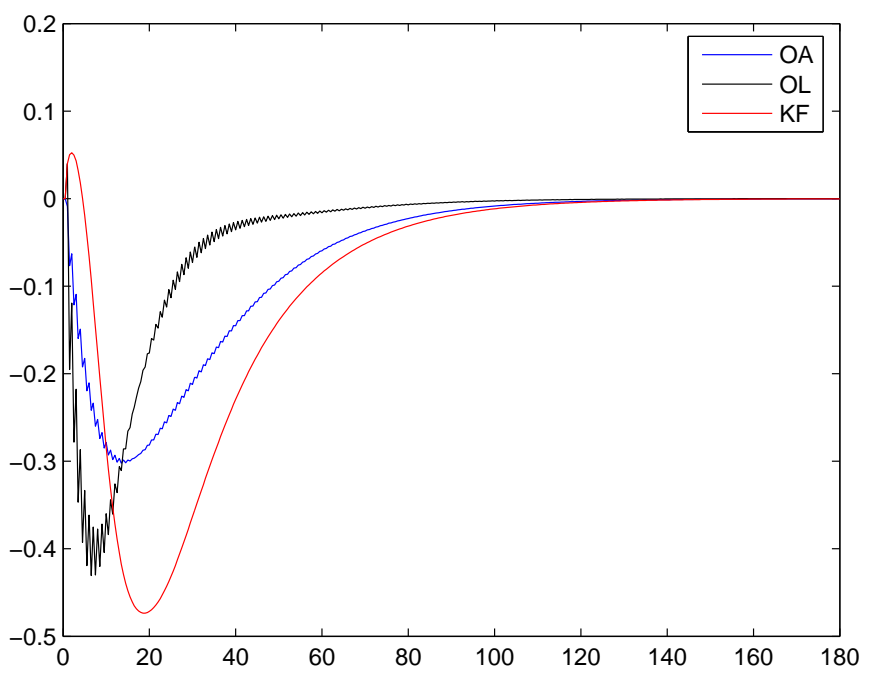

Figura 5.1. Evolución del error de estimación de la biomasa

con un error elevado, cuyo valor se reduce a lo largo del tiempo, logrando la convergencia del valor estimado con el valor real. Analizando el error presente, la siguiente tabla muestra los valores obtenidos:

\begin{tabular}{c|c|c} 
Observador & DEM & ECM \\
\hline Luenberger empleando LMI & 0.0961 & 0.0112 \\
Asintótico & 0.0942 & 0.0133 \\
Kalman & 0.1429 & 0.0289
\end{tabular}

Los valores de la desviación estándar de la media (DEM) y el error cuadrático medio (ECM) del observador Luenberger empleando LMI y el observador 
asintótico son muy cercanos entre sí, como lo denota la eficiencia relativa entre ellos $\left(E f \operatorname{Rel}_{O L / O A}=0,8408\right)$, indicando entonces que el comportamiento de ambos es muy similar.

A diferencia de lo que ocurre con el filtro Kalman el cuál presenta una mayor dispersión y la eficiencia relativa $E f \operatorname{Re}_{O L / K F}=0,3868$ indica que el observador Luenberger tiene un mejor comportamiento.

$\mathrm{Al}$ simular que existe un error en los parámetros del modelo $\left(\mu_{m}=0,3 h^{-1}\right.$, en lugar del 0.33) el observador Luenberger y el filtro Kalman presentan una mayor desviación en relación al comportamiento del observador asintótico, ya que los primeros son más dependientes de la exactitud del modelo (ver figura 5.2).

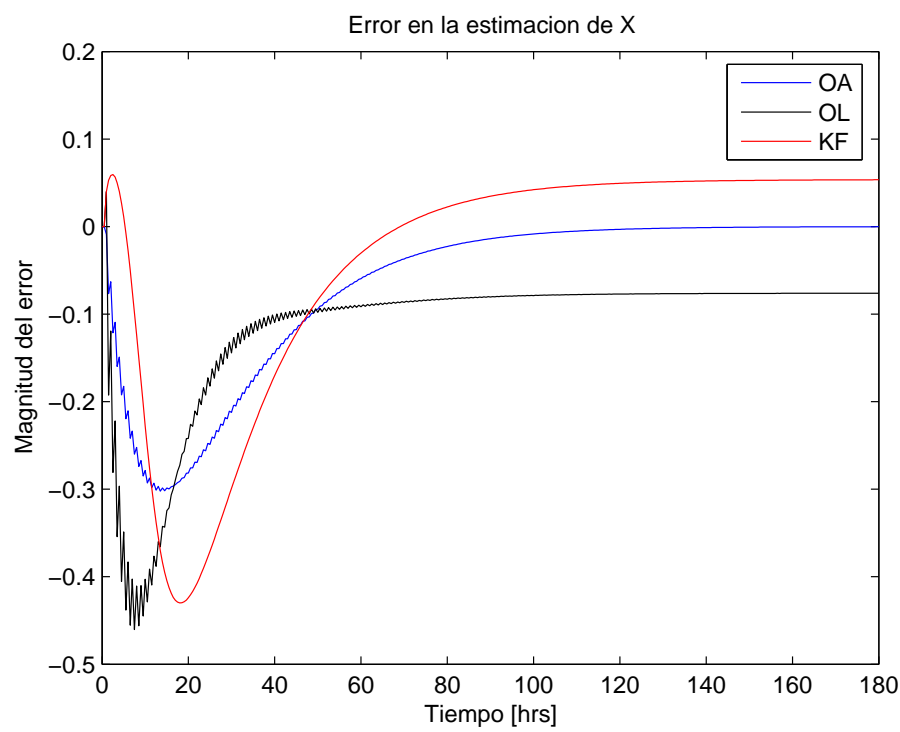

Figura 5.2. Evolución del error de estimación de la biomasa cuando se simula que $\mu_{m}=0,3 h^{-1}$

\subsection{Modelo de fermentación - Sistema productor metabólico}

En Shimizu [1996] se clasifican a los procesos metabólicos en tres tipos desde un punto de vista cinético:

Tipo I. Productos asociados al crecimiento alcanzado directamente de la energía metabólica del carbohidrato suministrado. 
Tipo II. Productos indirectos del metabolismo del carbohidrato.

Tipo III. Productos aparentemente no relacionados a la oxidación del carbohidrato.

Ejemplos de metabolitos de tipo I son el etanol y el ácido láctico, del tipo II el ácido cítrico y el acido salicílico y del tipo III el ácido L-glutámico y la producción de metabolitos secundarios como los antibióticos. En esta sección se empleará el modelo matemático de un sistema productor metabólico tipo I, y se simulará su comportamiento en Matlab para aplicar los desarrollos de estimadores descritos anteriormente.

\subsubsection{Descripción del modelo}

Si consideramos un cultivo simple de microorganismos que involucra una biomasa $(\mathrm{X})$ creciendo en un sustrato $(\mathrm{S})$ y produciendo un solo producto (P). El bioproceso se supone continuo con una tasa de disolución (D) y un sustrato de entrada con concentración $S_{i}$ [Farza et ${ }^{\sim}$ al., 1997].

$$
\begin{aligned}
\dot{X} & =\mu X-D X \\
\dot{S} & =-k_{1} \mu X-k_{2} \nu X+D\left(S_{i}-S\right) \\
\dot{P} & =\nu X-D P
\end{aligned}
$$

donde $k_{1}$ y $k_{2}$ son coeficientes de producción, $\mu$ y $\nu$ son la tasa específica de crecimiento y la tasa específica de biosíntesis, respectivamente, definidas por :

$$
\begin{array}{r}
\mu=\mu_{\max } \frac{S}{K_{S_{1}}+S+\frac{S^{2}}{K_{I}}} \\
\nu=\nu_{\max } \frac{S}{K_{S_{2}}+S} \frac{K_{P}}{K_{P}+P}
\end{array}
$$

donde $K_{S_{1}}$ y $K_{S_{2}}$ son constantes de saturación y $K_{I}$ y $K_{P}$ son constantes de inhibición.

La tabla 5.1 muestra los valores empleados para la simulación de este modelo. La tasa de disolución D es una señal trapezoidal de 0.1 a 0.2 l/h y el com-

Cuadro 5.1. Parámetros de simulación

\begin{tabular}{|l|l|l|l|l|l|l|}
\hline$k_{1}$ & 5 & $K_{S_{1}}$ & $1 g / l$ & $\mid S_{i}$ & $45 g / l$ \\
\hline$k_{2}$ & 10 & $K_{S_{2}}$ & $5 g / l$ & $X(0)$ & $0,5 g / l$ \\
\hline$\mu_{\max }$ & $0,25 h^{-1}$ & $K_{I}$ & $150(g / l)^{2}$ & $S(0)$ & $40 g / l$ \\
\hline$\nu_{\max }$ & $0,1 h^{-1}$ & $K_{P}$ & $10 g / l$ & $P(0)$ & $0,25 g / l$ \\
\hline
\end{tabular}

portamiento de la biomasa, substrato, producto y tasas de crecimiento se muestran en la figura 5.3. 

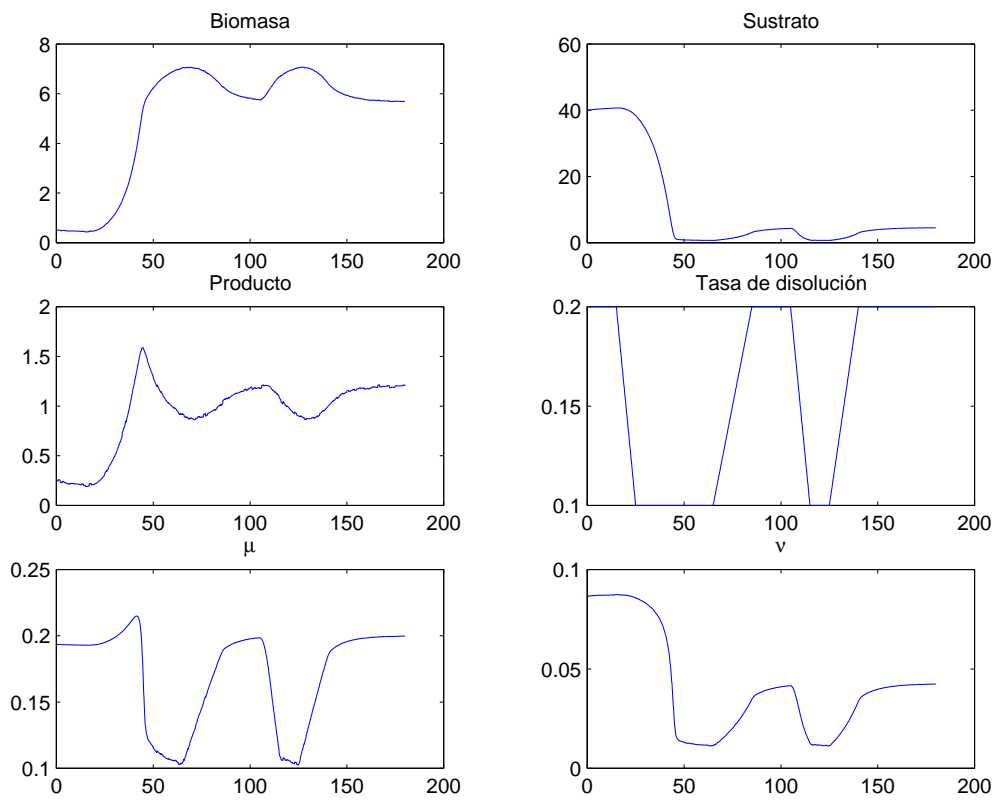

Figura 5.3. Simulación del bioproceso

Expresando la ecuación que define al bioproceso con el modelo dinámico general en forma matricial, ecuación (2.2), tendremos el sistema de ecuaciones que describe el bioproceso :

$$
\left[\begin{array}{c}
\dot{X} \\
\dot{S} \\
\dot{P}
\end{array}\right]=\left[\begin{array}{cc}
1 & 0 \\
-k_{1} & -k_{2} \\
0 & 1
\end{array}\right]\left[\begin{array}{l}
\mu X \\
\nu X
\end{array}\right]-D\left[\begin{array}{c}
X \\
S \\
P
\end{array}\right]+\left[\begin{array}{c}
0 \\
D S_{i} \\
0
\end{array}\right]
$$

Para efectos de este análisis, consideraremos que se disponen de las medidas del flujo de entrada y de la concentración del substrato de entrada, tal que $u=F(\xi)$ es conocido.

\subsubsection{Análisis de observabilidad}

Para la aplicación del observador de estados es indispensable que se cumplan con las condiciones de observabilidad para garantizar la convergencia del algoritmo. Para el análisis de observabilidad, empleamos la condición de observabilidad exponencial, [Bastin and Dochain, 1990]:

$$
\operatorname{rank}(\mathcal{O})=\operatorname{rank}(K)=r_{k}
$$

donde $\mathcal{O}$ es la matriz de observabilidad: 


$$
\mathcal{O}=\left[\begin{array}{c}
L \\
L A(\xi) \\
\vdots \\
L A(\xi)^{r_{k}-1}
\end{array}\right]
$$

y L es la matriz que selecciona los componentes medidos de $\xi$, para este modelo linealizando alrededor de $e=0$ :

$$
A(\xi)=\left[\begin{array}{cc}
1 & 0 \\
-k_{1} & -k_{2} \\
0 & 1
\end{array}\right]\left[\begin{array}{ccc}
\hat{\varphi}_{1 x} & \hat{\varphi}_{1 s} & 0 \\
\hat{\varphi}_{2 x} & \hat{\varphi}_{2 s} & \hat{\varphi}_{2 p}
\end{array}\right]-D I_{r_{k}}
$$

Las Jacobiana de las tasas de reacción son:

$$
\begin{aligned}
& \hat{\varphi}_{1 x}=\frac{\partial \mu(\xi) X}{\partial X}=\frac{\mu_{\max } S K_{I}}{K_{S 1} K_{I}+S K_{I}+S^{2}} \\
& \hat{\varphi}_{1 s}=\frac{\partial \mu(\xi) X}{\partial S}=-\frac{\mu_{\max } X K_{I}\left(-K_{S 1} K_{I}+S^{2}\right)}{\left(K_{S 1} K_{I}+S K_{I}+S^{2}\right)^{2}} \\
& \hat{\varphi}_{2 x}=\frac{\partial \nu(\xi) X}{\partial X}=\frac{\nu_{\max } S K_{P}}{\left(K_{S 2}+S\right)\left(K_{P}+P\right)} \\
& \hat{\varphi}_{2 s}=\frac{\partial \nu(\xi) X}{\partial S}=\frac{\nu_{\max } X K_{P} K_{S 2}}{\left(K_{S 2}+S\right)^{2}\left(K_{P}+P\right)} \\
& \hat{\varphi}_{2 p}=\frac{\partial \nu(\xi) X}{\partial P}=-\frac{\nu_{\max } S X K_{P}}{\left(K_{S 2}+S\right)\left(K_{P}+P\right)^{2}}
\end{aligned}
$$

Realizando el análisis considerando la medida de individual de cada una de las concentraciones:

\begin{tabular}{|c|c|}
\hline Medida & $\operatorname{rank}(\mathcal{O})$ \\
\hline $\mathrm{X}$ & 3 \\
$\mathrm{~S}$ & 3 \\
$\mathrm{P}$ & 3 \\
\hline
\end{tabular}

Por lo tanto, realizando la medida de cualquiera el sistema es globalmente observable. Sin embargo, si realizamos un análisis de observabilidad local, con el sistema no lineal redefinido como:

$$
\begin{aligned}
& {\left[\begin{array}{c}
\dot{X} \\
\dot{S} \\
\dot{P}
\end{array}\right]=\left[\begin{array}{cc}
1 & 0 \\
-k_{1} & -k_{2} \\
0 & 1
\end{array}\right]\left[\begin{array}{l}
\mu X \\
\nu X
\end{array}\right]+\left(\left[\begin{array}{c}
0 \\
S_{i} \\
0
\end{array}\right]-\left[\begin{array}{c}
X \\
S \\
P
\end{array}\right]\right) D} \\
& \xi_{1}=L\left[\begin{array}{c}
X \\
S \\
P
\end{array}\right]
\end{aligned}
$$

El cuál es orden $n=3$ y en forma general definido por: 


$$
\begin{aligned}
& \dot{x}=f(x)+g(x) u \\
& y=h(x)
\end{aligned}
$$

la observabilidad local de este sistema se define por:

$$
\operatorname{rank}(\mathcal{O L})=\operatorname{rank}\left\{d h, d L_{f} h, d L_{g} h, d L_{f}^{2} h, d L_{g}^{2} h\right\}^{T}=3
$$

donde:

$$
\begin{aligned}
& L_{f} h:=\frac{\partial h}{\partial x} f \quad L_{g} h:=\frac{\partial h}{\partial x} g \\
& L_{f}^{2} h:=\frac{\partial L_{f} h}{\partial x} f \quad L_{g}^{2} h:=\frac{\partial L_{g} h}{\partial x} g
\end{aligned}
$$

Realizando el análisis considerando que se cuenta con la medida individual de cada uno de los estados, la observabilidad local que se tendrá se muestra en la siguiente tabla:

\begin{tabular}{|c|c|}
\hline Medida & $\operatorname{rank}(\mathcal{O L})$ \\
\hline $\mathrm{X}$ & 2 \\
$\mathrm{~S}$ & 3 \\
$\mathrm{P}$ & 3 \\
\hline
\end{tabular}

De manera tal que la medida unicamente de la concentración de la Biomasa no garantiza la observabilidad del sistema. Para efectos de este ejemplo, se considerará que se cuenta la medida de la concentración de la Biomasa y del Producto, para realizar la estimación del Sustrato.

\subsubsection{Análisis de las Jacobianas}

Como se mostró en la sección 2.4.1, uno de los inconvenientes del observador Luenberger se presenta cuando el valor del sustrato tiende a valores muy pequeños, lo cuál puede producir falta de convergencia del observador debido a que los valores de las ganancias se elevan demasiado.

Para analizar este efecto en el modelo de fermentación propuesto, analizamos los límites de la Jacobiana de $\varphi(\xi)$, cuando el sustrato estimado tiende a un valor pequeño:

$$
\lim _{S \rightarrow 0}\left[\begin{array}{c}
\frac{\partial \varphi_{1}}{\partial \xi} \\
\frac{\partial \varphi_{2}}{\partial \xi}
\end{array}\right]=\left[\begin{array}{ccc}
0 & \frac{\mu_{\max } X}{K_{S 1}} & 0 \\
0 & \frac{\nu_{\max } X K_{P}}{K_{S 2}\left(K_{P}+P\right)} & 0
\end{array}\right]
$$

Donde se observa que $\hat{\varphi}_{1 x}, \hat{\varphi}_{2 x}, \hat{\varphi}_{2 p}$ tienden a cero cuando el sustrato lo hace, esto producirá que los valores de $\Omega(\xi)$ sean demasiado grandes. Los límites de la matriz $A(\xi)$ se definen entonces como:

$$
\lim _{S \rightarrow 0} A(\xi)=\left[\begin{array}{ccc}
-D & \hat{\varphi}_{1 s} & 0 \\
0 & -k 1 \hat{\varphi}_{1 s}-k 2 \hat{\varphi}_{2 s}-D & 0 \\
0 & \hat{\varphi}_{2 s} & -D
\end{array}\right]
$$


Esto puede provocar falta de convergencia de un observador Luenberger cuyas ganancias se determinen mediante un método clásico. Así, la estimación del Sustrato se presenta en esta sección bajo dos esquemas:

- Empleando un Observador Luenberger extendido cuyas ganancias son determinadas mediante el procedimiento LMI .

- Definiendo un observador híbrido, Observador Luenberger Extendido con un Observador Asintótico de Lazo Cerrado, cuya matriz de ganancia es determinada empleando LMI.

\subsubsection{Observador Luenberger empleando LMI}

A continuación se muestra la aplicación del desarrollo del observador Luenberger extendido resuelto mediante DMVT, y cuyas ganancias son determinadas empleando el procedimiento LMI. Los resultados se comparan con un filtro Kalman extendido y un observador asintótico de lazo abierto. El observador para el proceso de la ecuación (5.26) se define como:

$$
\begin{aligned}
& \dot{\hat{\xi}}=K \varphi(\hat{\xi})-D \hat{\xi}+u+\gamma L\left[\xi_{1}-\hat{\xi_{1}}\right] \\
& y=L \xi
\end{aligned}
$$

donde $L=\left[\begin{array}{lll}1 & 0 & 1\end{array}\right]$ y $u=F-Q$. La dinámica del error para este observador queda definida mediante la ecuación (2.16) y resuelta mediante el Teorema del Valor Medio Diferencial :

$$
\dot{e}(t)=(\mathcal{A}(h(t))-\gamma L) e(t)
$$

donde la matriz $\mathcal{A}$ se define por:

$$
\begin{gathered}
\mathcal{A}(h(t))=\sum_{i, j=1}^{m, n} h_{i j}(t) K \epsilon_{m}(i) \epsilon_{n}^{T}(j)-D I_{n} \\
\text { y } m=2, n=3 \text { y } h_{i j}=\left[\begin{array}{l}
h_{1 j} \\
h_{2 j}
\end{array}\right]=\left[\begin{array}{l}
\frac{\partial \mu(S) X}{\partial \xi_{j}} \\
\frac{\partial v(S) X}{\partial \xi_{j}}
\end{array}\right], j=1, \ldots, n
\end{gathered}
$$

Dado que $S_{\max }=S_{i n}$, entonces los límites de las concentraciones son: $X=\{0,9\} g / l, S=\{0,45\} g / l, P=\{0,4,5\} g / l$ Los valores máximos y mínimos de $h_{i}$ son:

$$
\left.\begin{array}{l}
\underline{h}_{i j}=\left[\begin{array}{ccc}
0,0227 & 0,0207 & 0 \\
0,0019 & 0,0019 & -9,5 x 10^{-8} \\
0,1891 & -0,0079 & 0 \\
0,0621 & 0,0012 & -0,0385
\end{array}\right] \\
\bar{h}_{i j}
\end{array}\right]
$$

El LMI

$$
\left\{\begin{array}{c}
\mathcal{A}(h(t))^{T} P-C^{T} R+P \mathcal{A}(h(t))-R^{T} C<0 \\
P>0
\end{array}\right.
$$


donde $h(t)=\left(\underline{h}_{i}, \bar{h}_{i}\right), R=\gamma^{T} P$ y $P=P^{T}$, es factible de acuerdo al desarrollo empleando Matlab, y su solución proporciona los valores de la matriz de ganancia:

$$
\gamma=[0,9736-1,5926-0,1314]^{T}
$$

\subsubsection{Observador asintótico}

Para realizar en este caso la estimación de $\mathrm{S}$ se emplea un observador asintótico

$$
\begin{aligned}
& \dot{\hat{Z}}=-D \hat{Z}+D \xi_{i 2} \\
& \hat{\xi}_{2}=A_{2}^{-1}\left(\hat{Z}-A_{1} \xi_{1}\right)
\end{aligned}
$$

para el cuál se considera la siguiente división de los estados:

$$
\begin{aligned}
\xi_{1} & =\left[\begin{array}{ll}
X & P
\end{array}\right]^{T} & \xi_{2} & =S \\
K_{1} & =\left[\begin{array}{ll}
1 & 0 \\
0 & 1
\end{array}\right] & & K_{2}=\left[-k_{1}-k_{2}\right] \\
A_{1} & =-\frac{K_{2}}{K_{1}} & & A_{2}=1
\end{aligned}
$$

\subsubsection{Resultados}

La figura 5.4 muestra el error relativo empleando el desarrollo del observador Luenberger que emplea como matriz de ganancia los resultados obtenidos mediante el LMI, y lo compara con las estimaciones resultantes al emplear un observador asintótico y un filtro Kalman.

La tabla siguiente muestra los resultados la desviación de la media (DEM) y el error cuadrático medio (ECM) del observador Luenberger empleando LMI, observador asintótico y del filtro Kalman.

En estos resultados se observa que el observador Luenberger presenta una menor dispersión de sus datos, representando con ello una mayor convergencia. La eficiencia relativa con respecto a los otros dos esquemas es menor a 1, indicando con ello que, bajo las condiciones de simulación, el observador Luenberger que emplea LMI para la solución de la matriz de ganancia es más eficiente, aun cuando en relación al filtro Kalman solamente es de un $60 \%$.

\begin{tabular}{c|c|c|c} 
Observador & DEM & ECM & Eficiencia relativa \\
\hline Luenberger empleando LMI & 0.1127 & 0.0144 & \\
Asintótico & 0.3299 & 0.1178 & 0.1222 \\
Kalman & 0.1491 & 0.0241 & 0.5981
\end{tabular}

La figura 5.5 muestra la evolución del error cuando se simula que existe la presencia de ruido en la medida. Se observa que el observador asintótico no 


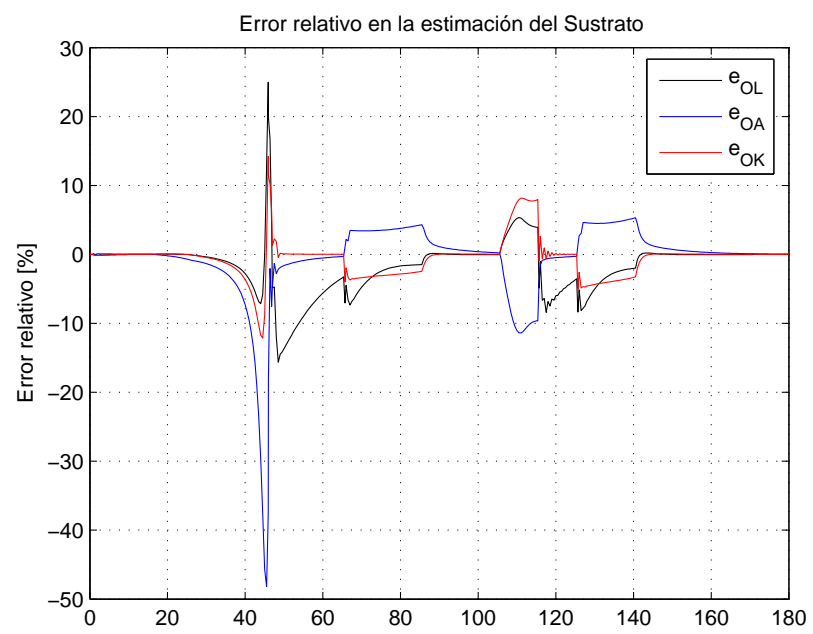

Figura 5.4. Comportamiento del error relativo en la estimación del sustrato. $e_{O L}$ : error empleando un observador Luenberger, $e_{O A}$ : error empleando un Observador Asintótico, $e_{O K}$ : error empleando un Filtro Kalman

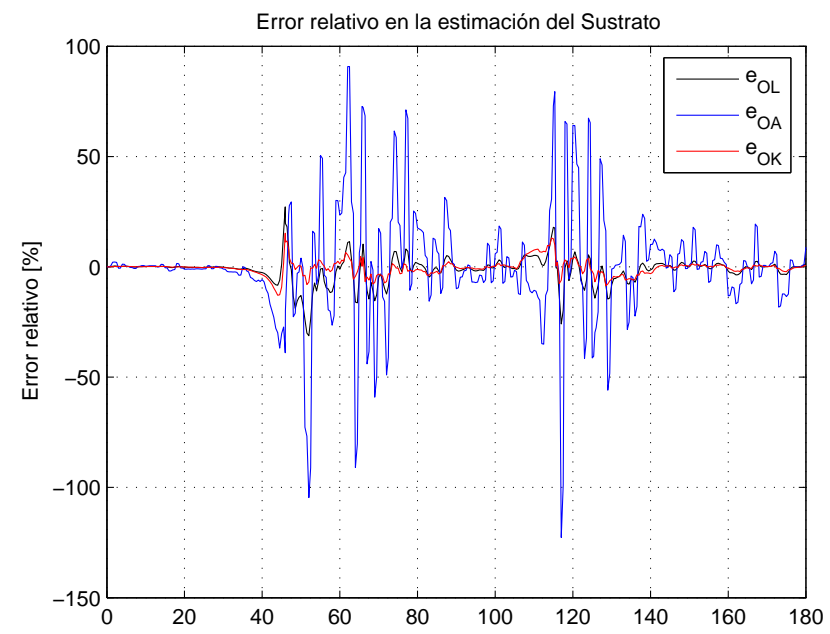

Figura 5.5. Evolución del error relativo de observación ante la presencia de ruido en la medida

presenta una buena respuesta ante la presencia de ruido.

Por el contrario, el observador Luenberger empleando LMI y el filtro Kalman tiene una menor desviación ante la presencia del ruido, como se observa en su desviación estándar de la media, indicando con ello que, ante el error si- 
mulado, ambos son más eficiente en relación con el observador asintótico.

\begin{tabular}{c|c|c|c} 
Observador & DEM & ECM & Eficiencia relativa \\
\hline Luenberger empleando LMI & 0.1352 & 0.0196 & \\
Asintótico & 0.4997 & 0.2555 & 0.0769 \\
Kalman & 0.1556 & 0.0258 & 0.76
\end{tabular}

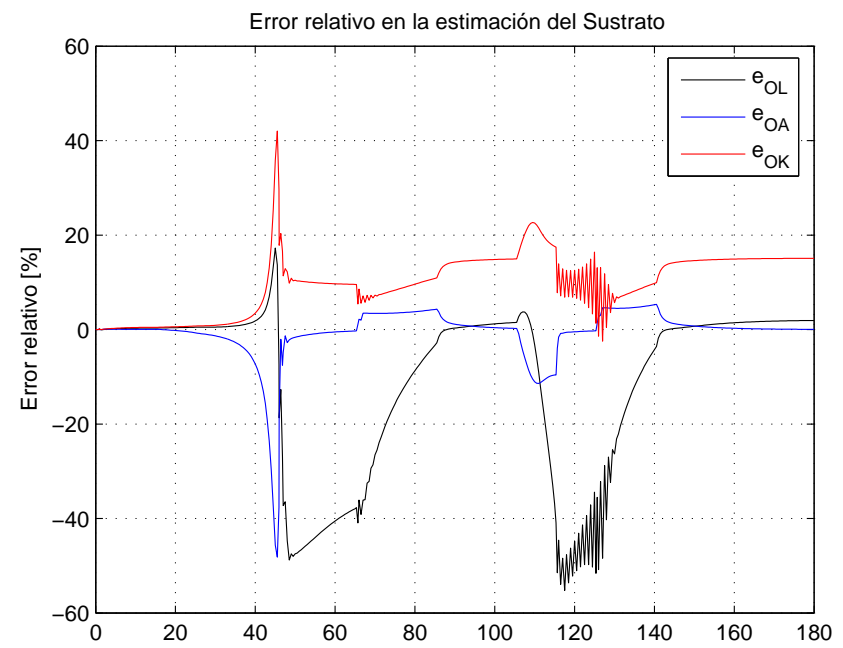

Figura 5.6. Evolución del error relativo de observación cuando existe variación en el parámetro $\mu_{\max }$

La figura 5.6 muestra la evolución del error cuando se simula que existe una variación en el parámetro $\mu_{\max }=0,27 h^{-1}$. Se observa que el observador asintótico no presenta una gran afectación por variaciones en los parámetros $(\mathrm{ECM}=0.1178)$, sin embargo el filtro Kalman es quien presenta una mayor dispersión $(\mathrm{ECM}=0.1802)$ debido a su dependencia de la exactitud del modelo.

El observador Luenberger empleando LMI presenta un mejor comportamiento que el de Kalman ya que la ganancia fue determinada para un intervalo de variación en los parámetros $(\mathrm{ECM}=0.0447)$.

Por último, se simula el comportamiento de los tres esquemas de observadores, si se cambia el tipo de entrada, es decir, en lugar de que la tasa de disolución sea de tipo trapezoidal, se cambia a un escalón que varía de 0.1 a $0.2 \mathrm{l} / \mathrm{h}$. Los resultados se muestran en la figura 5.7.

$\mathrm{Al}$ analizar la eficiencia relativa entre el observador Luenberger empleando LMI y el observador asintótico $\left(E f_{r e l}=0,2873\right)$ se observa que el primero es 


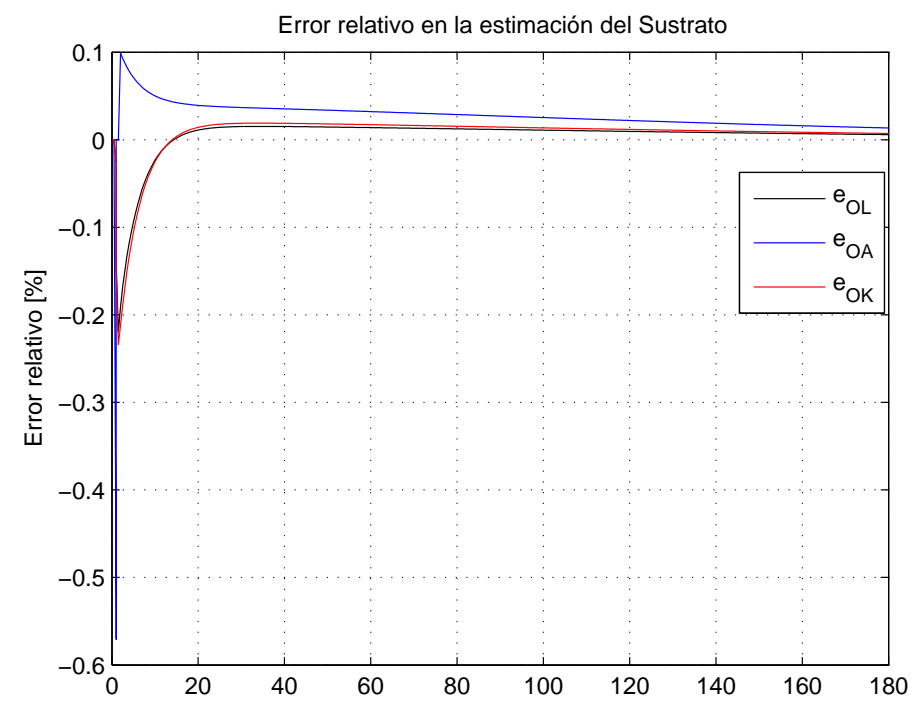

Figura 5.7. Evolución del error relativo de observación cuando la tasa de disolución D es un escalón de 0.1 a $0.2 \mathrm{l} / \mathrm{h}$

más eficiente, lo cuál se comprueba gráficamente. Así mismo, en relación con el filtro Kalman $\left(E f_{\text {rel }}=0,7850\right)$ el desempeño es similar, dado que ambos no son afectados por el cambio de $\mathrm{D}$, a diferencia de lo que sucede con el observador asintótico.

\subsection{Observador híbrido empleando LMI}

En esta sección se emplea el procedimiento de calculo de la ganancia mediante LMI y se aplica a un observador híbrido, compuesto de un observador Luenberger extendido y un observador asintótico de lazo cerrado. Como se explicó anteriormente, la sintonización se realiza ajustando a $\delta=1$, es decir el observador híbrido se comporta como un observador Luenberger, de manera tal que el observador se define por:

$$
\left\{\begin{array}{l}
\dot{\hat{\xi}}_{1}=K_{1} \varphi(\hat{\xi})-D \hat{\xi}_{1}+u_{1}+\gamma_{1}(\hat{\xi})\left(\xi_{1}-\hat{\xi}_{1}\right) \\
\dot{\hat{Z}}=-D \hat{Z}+A_{1} u_{1}+A_{2} u_{2}+\gamma_{z}(\hat{\xi})\left(\xi_{1}-\hat{\xi}_{1}\right) \\
\left.\hat{\xi}_{2}=A_{2}^{+}\left(\hat{Z}-A_{1} \hat{\xi}\right)\right)
\end{array}\right.
$$

Esta estructura es idéntica a la mostrada en la sección 4.4.1, de manera que la solución de la matriz de ganancia $\mathcal{G}$ mediante LMI es: 


$$
\mathcal{G}=\left[\begin{array}{ccc}
0,6673 & 0 & 0 \\
-1,4436 & 0 & 0 \\
-0,0417 & 0 & 0
\end{array}\right]
$$

Aplicando este esquema de observador híbrido al mismo sistema anterior, la figura 5.8 muestra la comparación de los errores relativos, expresado en porcentaje, entre este observador, el observador Luenberger empleando LMI, y el observador asintótico.

Como se observa, el observador híbrido y el observador asintótico tienen un

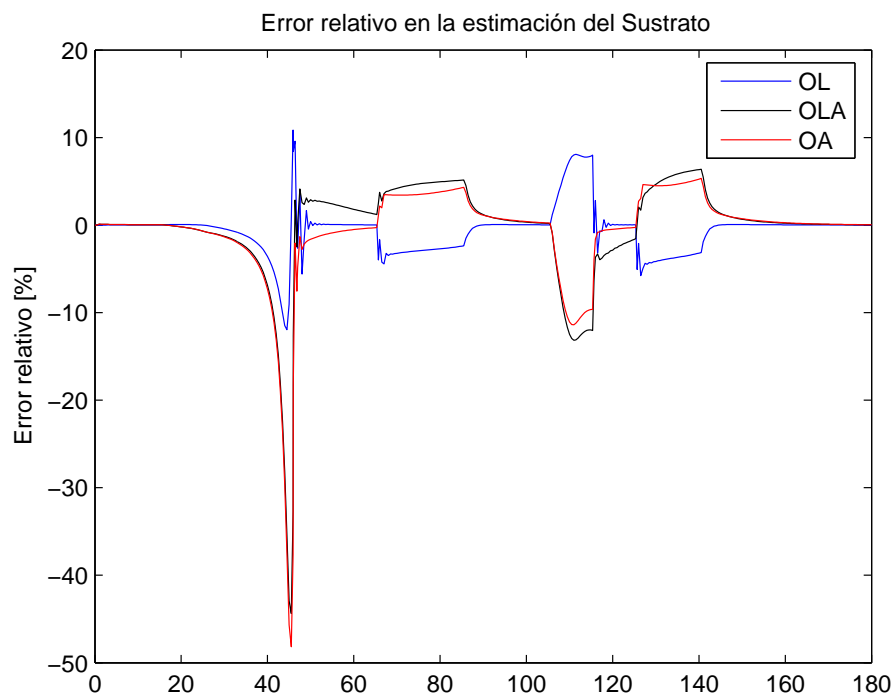

Figura 5.8. Evolución del error relativo de observación. Error del observador Luenberger extendido empleando LMI $(O L)$. Error del observador híbrido empleando LMI $(O L A)$. Error del observador asintótico $(O A)$

comportamiento similar, debido a la aportación a la convergencia del observador por por parte de ambos tipos. Esto debido al parámetro $\delta$, el cuál, en base al error en la medida, oscila entre un observador asintótico (si el error es cercano a cero) y un observador Luenberger (cuando el error es grande). Este comportamiento se observa en la figura 5.9.

Al comparar el comportamiento de estos observadores empleando el error cuadrático medio (ECM), siendo estos: $E C M_{O L}=0,0231, E C M_{O L A}=$ 0,1072 y $E C M_{O A}=0,1185$ se observa que el observador híbrido empleando LMI y el observador asintótico tiene un comportamiento similar, ambos peor que el observador Luenberger empleando LMI.

El uso del procedimiento LMI para la determinación de las ganancias del 


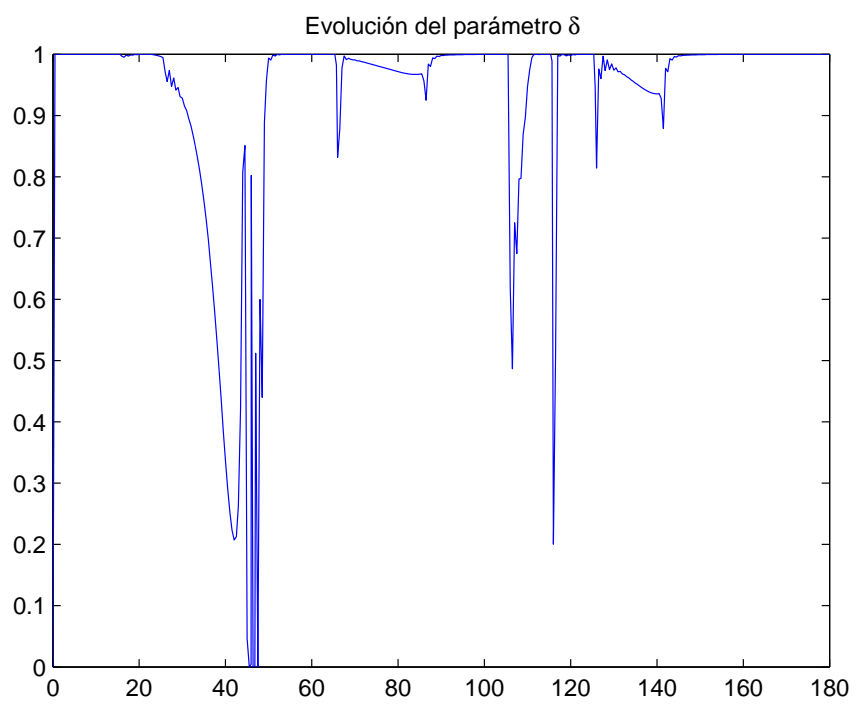

Figura 5.9. Evolución del parámetro $\delta$

observador nos permite garantizar la estabilidad del sistema dentro de los vértices del politopo definido, independientemente del esquema del observador.

Sin embargo, si existen variaciones en los parámetros, por ejemplo $\mu_{\max }=$ $0,27 h^{-1}$, el observador híbrido mejora el rendimiento del observador Luenberger, dado que su componente relativa al observador asintótico que no depende de estas variaciones permite mejorar su convergencia.

La figura 5.10 muestra este comportamiento, indicando también los valores del error cuadrático medio para cada uno de estos observadores.

En el análisis del error cuadrático medio se obtienen los siguientes valores:

- $E C M_{O A}=0,1185 \mathrm{~g} / \mathrm{l}$

- $E C M_{O H}=0,1381 \mathrm{~g} / \mathrm{l}$

- $E C M_{O L}=0,1582 g / l$

observándose que los valores son muy próximos entre sí, siendo el ECM del observador híbrido mejor que el observador Luenberger, debido a la incorporación del observador asintótico.

\subsection{Modelo de fermentación - Sistema multifrecuencial}

En esta sección aplicaremos los conceptos desarrollados en el capítulo 4 para mostrar la operación del observador híbrido multifrecuencial, compuesto 


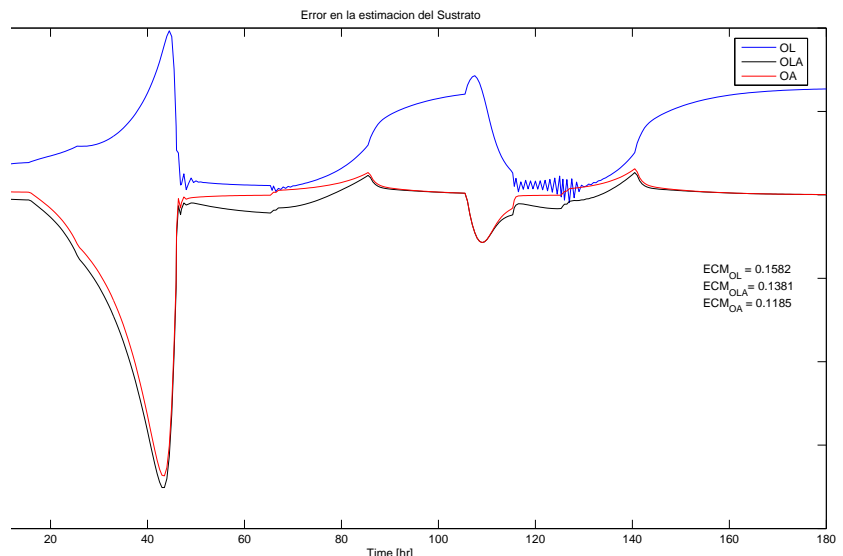

Figura 5.10. Evolución del error absoluto ante la presencia de variaciones en $\mu_{\max }$. Error del observador Luenberger extendido empleando LMI $(O L)$. Error del observador híbrido empleando LMI $(O L A)$. Error del observador asintótico $(O A)$

durante el periodo de tiempo en que se cuenta con las medidas continuas de un observador Luenberger extendido y un observador asintótico, y al disponer de las medidas esporádicas se realiza la corrección de la estimación. Consideremos el siguiente sistema, Picó [2002]:

$$
\left[\begin{array}{c}
\dot{X} \\
\dot{S} \\
\dot{E}
\end{array}\right]=\left[\begin{array}{c}
1 \\
-k_{s} \\
k_{e}
\end{array}\right] \varphi(\xi)-D\left[\begin{array}{c}
X \\
S \\
E
\end{array}\right]+\left[\begin{array}{c}
0 \\
D S_{i n} \\
0
\end{array}\right]
$$

donde:

$$
\varphi(\xi)=\frac{\mu_{m} S}{K_{S 1}+S} X
$$

y los valores de los coeficientes son : $k_{s}=6,2, k_{e}=3, K_{S 1}=0,043, \mu_{m}=$ 0,34. La figura 5.11 muestra los datos disponibles del modelo, empleando un sensor de biomasa desarrollado en la UPV y las medidas de sustrato y etanol realizadas fuera de línea y en forma esporádica.

La partición correspondiente es:

$$
\begin{aligned}
& \xi_{1}=X \quad u_{1}=0 \quad K_{1}=1 \\
& \xi_{2}=[S, E]^{T} u_{2}=\left[D S_{i n}, 0\right]^{T} K_{2}=\left[-k_{s}, k_{e}\right]^{T}
\end{aligned}
$$

Si definimos la variable auxiliar $Z \in \mathbb{R}^{n-q}$ como:

$$
\begin{aligned}
Z & =A_{1} \xi_{1}+A_{2} \xi_{2} \\
{\left[\begin{array}{c}
z_{1} \\
z_{2}
\end{array}\right] } & =\left[\begin{array}{c}
k_{s} \\
-k_{e}
\end{array}\right] X+[1]\left[\begin{array}{c}
S \\
E
\end{array}\right]
\end{aligned}
$$



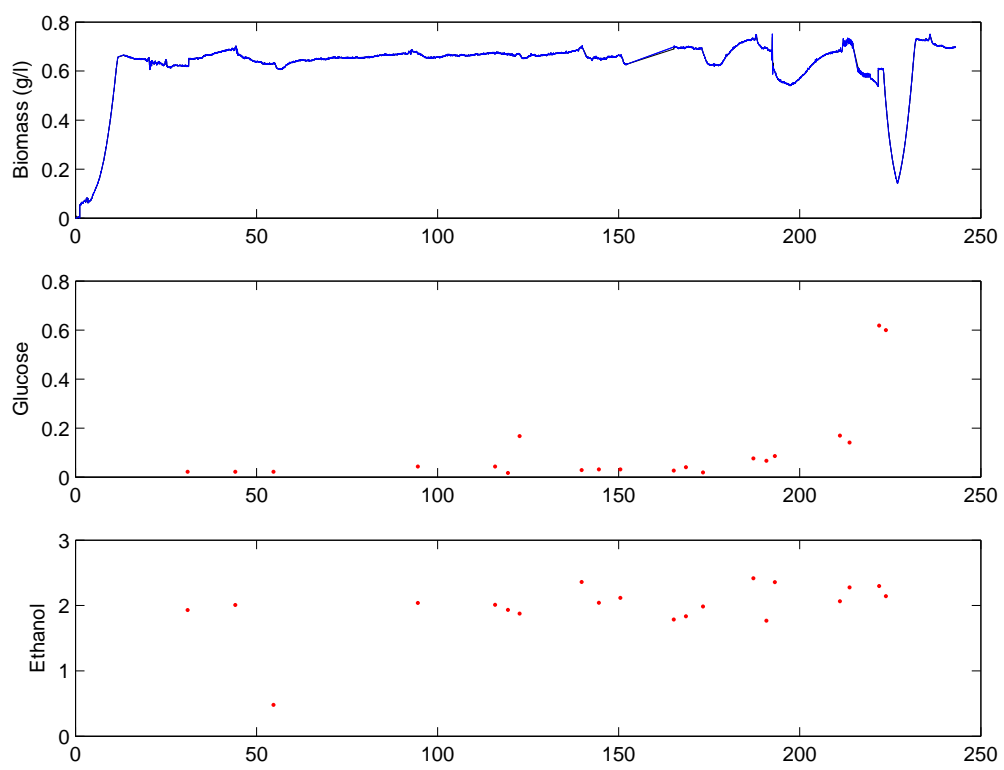

Figura 5.11. Información disponibles del modelo

Entonces el modelo se redefine como:

$$
\left\{\begin{array}{l}
\dot{\xi}_{1}=K_{1} \varphi\left(\xi_{1}, Z\right)-D \xi_{1} \\
\dot{Z}=-D Z+u_{2} \\
\xi_{2}=Z-A_{1} \xi_{1}
\end{array}\right.
$$

donde

$$
\varphi\left(\xi_{1}, Z\right)=\frac{\mu_{m}\left(z_{1}-k_{s} X\right)}{K_{S 1}+z_{1}-k_{s} X} X
$$

\subsubsection{Descripción del observador híbrido multifrecuencial}

El observador híbrido para el modelo definido en la ecuación 5.45 es:

$$
\left\{\begin{array}{l}
\dot{\hat{\xi}}_{1}=K_{1} \varphi\left(\hat{\xi_{1}}, \hat{Z}\right)-D \hat{\xi_{1}}+\gamma_{1}(y-\hat{y}) \\
\dot{\hat{Z}}=-D \hat{Z}+u_{2}+\gamma_{z} \delta(y-\hat{y}) \\
\hat{\xi}_{2}=\hat{Z}-A_{1}\left(\delta \hat{\xi}_{1}+(1-\delta) y\right)
\end{array}\right.
$$

A continuación se analizará el comportamiento del observador para cada momento, es decir, cuando se dispone de medidas continuas y cuando se cuenta con su valor medido fuera de línea (medidas esporádicas) definiendo el conjunto de ecuaciones de la dinámica del error para cada uno de los casos, 
ajustando el parámetro $\delta=1$ para realizar la sintonización.

Medidas continuas En este caso disponemos de las medidas en línea tal que $y=\xi_{1}$ y $\hat{y}=\hat{\xi}_{1}$

$$
\left\{\begin{array}{l}
\dot{e}_{1}=K_{1}\left\{\varphi\left(\xi_{1}, Z\right)-\varphi\left(\hat{\xi}_{1}, \hat{Z}\right)\right\}-D e_{1}-\gamma_{1} e_{1} \\
\dot{e}_{z}=-D e_{z}-\gamma_{z} e_{1}
\end{array}\right.
$$

donde $e_{1}=\xi_{1}-\hat{\xi}_{1}$ y $e_{z}=Z-\hat{Z}$.

Medidas esporádicas En este instante del tiempo, se cuenta con la medida de todos los estados del sistema, tal que $y=\xi$ y $\hat{y}=\hat{\xi}$, y la partición correspondiente será

$$
\left\{\begin{array}{l}
\dot{e}_{1}=K_{1}\left\{\varphi\left(\xi_{1}, Z\right)-\varphi\left(\hat{\xi_{1}}, \hat{Z}\right)\right\}-D e_{1}-\gamma_{1} e_{1} \\
\dot{e}_{z}=-D e_{z}-\gamma_{z} e_{z}
\end{array}\right.
$$

Aplicamos el Teorema del Valor Medio Diferencia (DMVT) para su resolución tendremos que:

$$
\begin{aligned}
\varphi\left(\xi_{1}, Z\right)-\varphi\left(\hat{\xi_{1}}, \hat{Z}\right)= & \left(\sum_{i, j=1}^{m, q} \epsilon_{m}(i) \epsilon_{q}^{T}(j) \frac{\partial \varphi_{i}\left(\xi_{1}^{*}, Z^{*}\right)}{\partial \xi_{1 j}}\right) e_{1}+ \\
& +\left(\sum_{i, j=1}^{m, n-q} \epsilon_{1}^{T}(i) \epsilon_{q}^{T}(j) \frac{\partial \varphi_{i}\left(\xi_{1}^{*}, Z^{*}\right)}{\partial Z_{j}}\right) e_{z}
\end{aligned}
$$

donde $m=1, q=1$ y $n-q=2$, entonces:

$$
\begin{aligned}
h_{11} & =\frac{\partial \varphi\left(\xi_{1}^{*}, Z^{*}\right)}{\partial \xi_{1}} \\
h_{z} & =\left[\begin{array}{ll}
\frac{\partial \varphi\left(\xi_{1}^{*}, Z^{*}\right)}{\partial Z_{1}} & \frac{\partial \varphi\left(\xi_{1}^{*}, Z^{*}\right)}{\partial Z_{2}}
\end{array}\right] \\
\mathcal{H}_{1} & =\epsilon_{1}(1) \epsilon_{2}^{T}(1) h_{11} \\
\mathcal{H}_{z} & =\epsilon_{1}(1) \epsilon_{2}^{T}(1) h_{z 1}+\epsilon_{1}(1) \epsilon_{2}^{T}(2) h_{z 2} \\
e_{z} & =\left[\begin{array}{ll}
e_{z 1} & e_{z 2}
\end{array}\right]^{T}
\end{aligned}
$$

la base canónica se define como: $\epsilon_{1}(1)=[1], \epsilon_{2}^{T}(1)=\left[\begin{array}{ll}1 & 0\end{array}\right]^{T}$ y $\epsilon_{2}^{T}(2)=$ $\left[\begin{array}{ll}0 & 1\end{array}\right]^{T}$ y los límites del vector $h(t)$ calculados con los límites de los estados $\underline{X}, \bar{X}, \underline{S}, \bar{S}$ у $\underline{P}, \bar{P}:$

$$
\begin{aligned}
& \alpha_{1} \in\left(\underline{h}_{11}, \bar{h}_{11}\right)=(-0,2585,0,3399) \\
& \alpha_{z} \in\left(\underline{h}_{z}, \bar{h}_{z}\right)=\left(\left[\begin{array}{ll}
0 & 0
\end{array}\right],\left[\begin{array}{ll}
0,0520 & 0
\end{array}\right]\right)
\end{aligned}
$$

Observador híbrido - medidas continuas El error de observación entonces se define como:

$$
\left(\mathcal{A}\left(\alpha_{1}, \alpha_{z}\right)-\mathcal{G}_{1}\right) \epsilon=\left[\begin{array}{cc}
\mathcal{H}_{1}\left(\alpha_{1}\right)-D I_{1} & \mathcal{H}_{z}\left(\alpha_{z}\right) \\
0 & -D I_{2}
\end{array}\right]-\left[\begin{array}{ccc}
\gamma_{1} & 0 & 0 \\
\gamma_{z 1} & 0 & 0 \\
\gamma_{z 2} & 0 & 0
\end{array}\right]\left[\begin{array}{c}
e_{1} \\
e_{z 1} \\
e_{z 2}
\end{array}\right]
$$


Resolviendo el LMI con Matlab:

$$
\left\{\begin{array}{l}
\mathcal{A}^{T}\left(\alpha_{1}, \alpha_{z}\right) P_{1}-R_{1}+P_{1} \mathcal{A}\left(\alpha_{1}, \alpha_{z}\right)-R_{1}^{T}<0 \\
P_{1}>0
\end{array}\right.
$$

Es factible, en $t_{\min }=-0,013623$ y la ganancia correspondiente es:

$$
\mathcal{G}_{1}=\left[\begin{array}{ccc}
0,9637 & 0 & 0 \\
0,0524 & 0 & 0 \\
0 & 0 & 0
\end{array}\right]
$$

Observador híbrido - medidas esporádicas El error de observación entonces se define como:

$$
\left(\mathcal{A}\left(\alpha_{1}, \alpha_{z}\right)-\mathcal{G}_{2}\right) \epsilon=\left[\begin{array}{rr}
\mathcal{H}_{1}\left(\alpha_{1}\right)-D I_{1} & \mathcal{H}_{z}\left(\alpha_{z}\right) \\
0 & -D I_{2}
\end{array}\right]-\left[\begin{array}{ccc}
\gamma_{1} & 0 & 0 \\
0 & \gamma_{z 1} & 0 \\
0 & 0 & \gamma_{z 2}
\end{array}\right]\left[\begin{array}{c}
e_{1} \\
e_{z 1} \\
e_{z 2}
\end{array}\right]
$$

Resolviendo el LMI con Matlab:

$$
\left\{\begin{array}{l}
\mathcal{A}^{T}\left(\alpha_{1}, \alpha_{z}\right) P_{2}-R_{2}+P_{2} \mathcal{A}\left(\alpha_{1}, \alpha_{z}\right)-R_{2}^{T}<0 \\
P_{2}>0
\end{array}\right.
$$

Es factible, en $t_{\min }=-0,90648$ y la ganancia es:

$$
\mathcal{G}_{2}=\left[\begin{array}{ccc}
1,138 & 0 & 0 \\
0 & 1,4721 & 0 \\
0 & 0 & 1,4713
\end{array}\right]
$$

\subsubsection{Resultados}

La aplicación de estas ganancias al observador dan lugar a los resultados mostrados en la figura 5.12. Se observa que el observador híbrido(línea negra) se ajusta a los valores del sustrato y etanol cuando estos están disponibles. Mientras no se cuente con esta información, sigue la trayectoria del observador asintótico (línea azul).

Con la finalidad de poder comparar ambos esquemas y observar el comportamiento durante la presencia del valor real de la glucosa y el etanol, la figura 5.13 muestra estos instantes, donde se observa que el observador asintótico presenta una mayor dispersión de sus datos respecto al observador híbrido empleando LMI. 

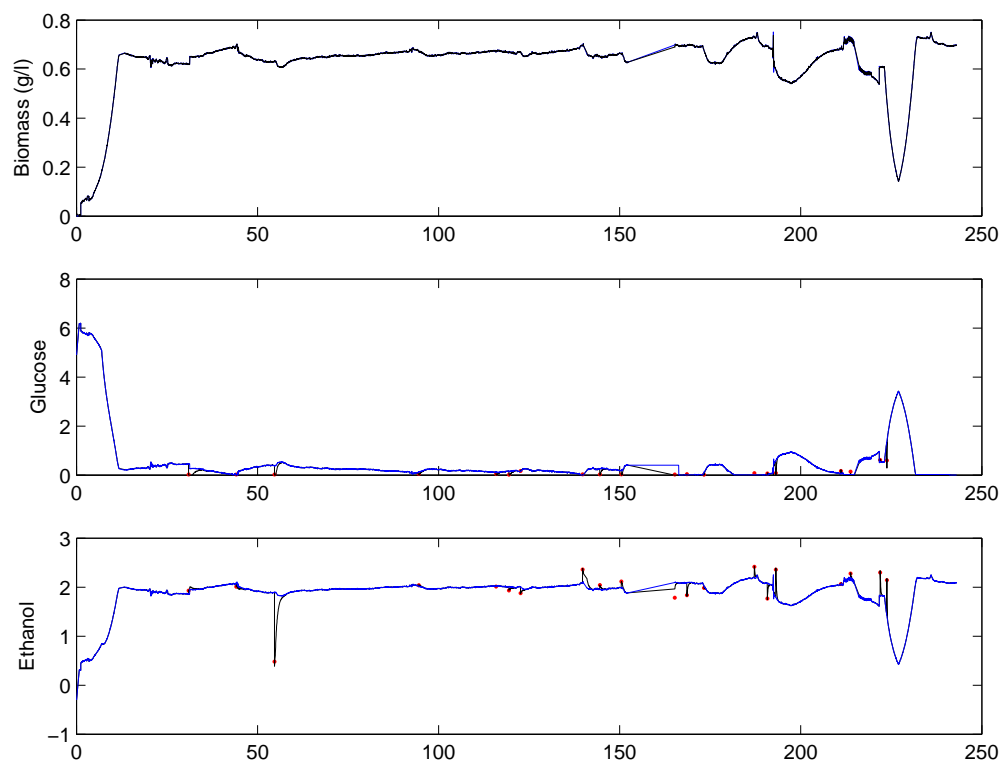

Figura 5.12. Estimación de Sustrato y Etanol empleando Observador Asintótico (línea azul) y un Observador Híbrido (línea negra)
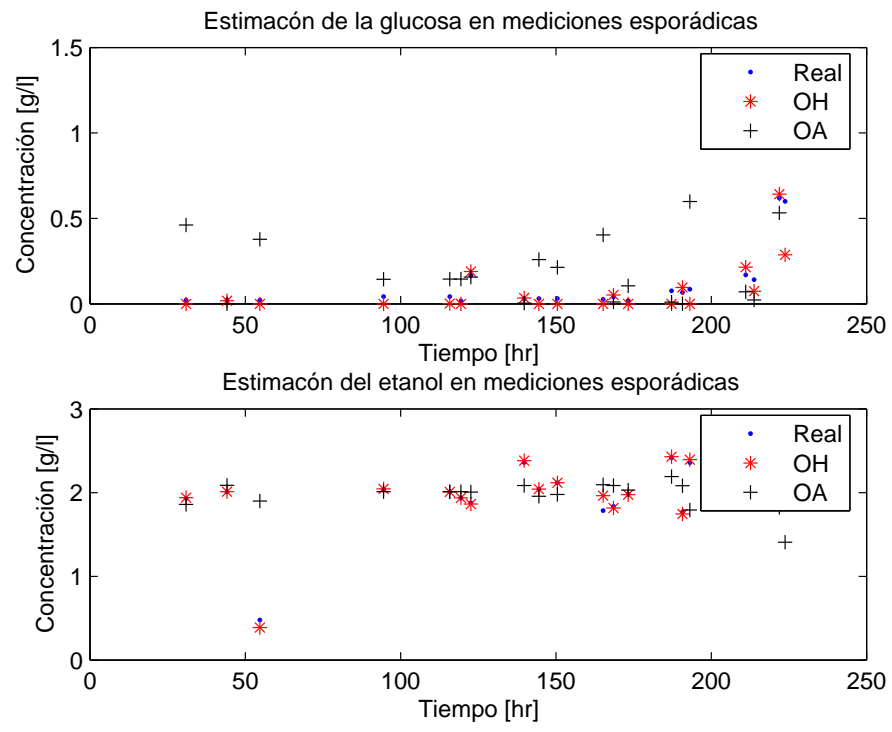

Figura 5.13. Valores de la medida y de la estimación en instantes esporádicos , (.) Medida real (+) Observador Asintótico, $(*)$ Observador Híbrido 
Si por precisión se entiende el promedio del grado de correspondencia entre pares individuales de valores medidos y observador, entonces empleamos el error cuadrático medio (ECM), el cuál proporciona la medida de las diferencias en promedio entre los valores medidos y observador:

\begin{tabular}{c|cc} 
Estado Estimado & Observador Asintótico & Observador Híbrido \\
\hline $\mathrm{S}$ & 0.0762 & 0.0063 \\
$\mathrm{E}$ & 0.1782 & 0.0023
\end{tabular}

Entonces podemos ver que el observador híbrido presenta valores menores al observador asintótico, indicando con ello que existe una mayor cercanía entre los valores individuales observados y los medidos del sustrato y etanol.

\subsection{Discusión}

Se han presentado dos tipos de modelos matemáticos para aplicar las variantes del observador para bioprocesos empleando LMI: observador Luenberger, observador híbrido y observador híbrido multifrecuencial. Estos casos se compararon con el observador asintótico mostrando contar con una mejor velocidad de convergencia y una mejor estimación, empleando para ello el error cuadrático medio (ECM).

Sin embargo, debido a que la matriz $\mathcal{A}(h)$ se determina en base a las Jacobianas del modelo de las tasas de reacción, esta dependencia provoca que exista cierta divergencia si se simulan cambios en los parámetros del modelo. Este inconveniente se ve reducido en el observador híbrido, el cuál aprovecha las ventajas del Luenberger y del asintótico, mejorando la respuesta del observador respecto al solo Luenberger ante este escenario de errores en el modelado. 



\section{Conclusiones y Trabajos futuros}

\subsection{Conclusiones}

El desarrollo de estimadores de estados es una área de gran desarrollo en el ámbito de la automatización y control debido a la falta de sensores que realicen la medida en línea de alguna variable de importancia para el esquema de control.

Esta tesis aborda el desarrollo de un observador de estado para bioprocesos basado en el modelo general para observadores teniendo como principal característica que la matriz de ganancia es determinada empleando el procedimiento de desigualdades matriciales lineales (LMI). En este sentido, se han cubierto el objetivo general y los objetivos particulares planteadas inicialmente.

Se ha propuesto, desarrollado y analizado tres esquemas del observador para bioprocesos empleando LMI:

Observador Luenberger. Este es el esquema fundamental del trabajo de tesis y se desarrolló en el capítulo 3, presentando como principales ventajas el considerar las incertidumbres en el modelo que permiten definir un politopo que se basa en los límites de operación de los estados del sistema.

Observador híbrido. Esta es una extensión del observador Luenberger anterior, ya que se le asocia con un observador asintótico de lazo cerrado, mejorando con ello la divergencia presentada ante variaciones del modelo fuera de los límites establecidos. La transición entre ambos algoritmos es determinado por un parámetros cuyo sintonización permite hacer uso de uno u otro esquema de acuerdo a la magnitud del error.

Observador híbrido multifrecuencial. Siendo este observador una extensión del observador híbrido, ambos analizados y desarrollados en el capítulo 4, aplicando el concepto a un sistema multifrecuencial, en el cuál se disponen de medidas continuas y medidas esporádicas, teniendo una mejor convergencia en su comparación con un observador asintótico con el modelo dinámico presentado.

En general, el observador propuesto presenta un mejor comportamiento también al compararse con un filtro Kalman extendido, ya que este último depende del modelo tangencial para la linealización, siendo entonces válido solamente localmente. Sin embargo, el desarrollo propuesto es válido en un 
politopo definido por los intervalos de operación, de manera tal que la definición de dicho politopo representa la principal tarea a realizar.

\subsection{Trabajos futuros}

El uso del procedimiento mediante LMI se ha extendido para aplicarse en todo aquel análisis que conlleve a una desigualdad y que se cumplan las condiciones de simetría en las matrices definidas.

En su aplicación en el diseño de observadores para bioprocesos, el presente trabajo establece un camino a seguir en este campo, quedando como posibles tareas futuras, extenderse para el desarrollo de:

- Estimadores basado en observador, que permita estimar el comportamiento de los parámetros del sistema basándose en el desarrollo del observador Luenberger empleando LMI y considerando los intervalos de operación de los parámetros para la definición del politopo.

- Observadores de alta ganancia, en el cuál se definan su ganancia empleando desigualdades matriciales y considerar el acotamiento del modelo dinámico general mediante los intervalos de operación.

- Analizar el comportamiento del algoritmo propuesto cuando el modelo del bioproceso no se conozca completamente, es decir que dicho modelo no sea perfecto y existan perturbaciones y ruidos en la medida.

- Analizar el comportamiento del parámetro $\delta$ del observador híbrido, empleando diversas estrategias que mejoren su convergencia en base a la magnitud del error en la medida, es decir que permita transitar de un algoritmo a otro si el error es "grande".

- Analizar otras alternativas del observador híbrido, por ejemplo el filtro Kalman-Asintótico y el observador de alta ganancia-asintótico, que contemplen la definición del politopo y la determinación de la ganancia mediante LMI.

- Analizar la robustez del algoritmo multifrecuencial para su aplicación a otros modelos de tasas de reacción, así como considerar los retrasos en la obtención de la medida. 


\section{Referencias}

T. Antczak. Mean value in inverxity analysis. Nonlinear Analysis, 60:14731484, 2005.

M. Arcak and P. Kokotović. Nonlinear observers: A circle criterion design. Proc. of the 38th Conf. on Decision and Control, pages 4872-4876, Dec. 1999.

M. Arnold and B.N. Datta. Single-input eigenvalue assignment algorithms: A close look. SIAM J. on Matrix Analysis and Applications, 19(2):444-467, 1998.

G.I. Bara, J. Daafouz, and F. Kratz. Advanced gain scheduling techniques for the design of parameter-dependent observers. Proc. of the 48th IEEE Conf. on Decision and Control, pages 3892-3897, Dec. 2001.

G. Bastin and D. Dochain. On-line estimation and adaptive control of bioreactors. Elsevier, Amsterdan, 1990.

G. Bastin and J.F. Van Impe. Nonlinear and adaptive control in biotechnology: A tutorial. European Journal of Control, 1(37):37-53, 1997.

O. Bernard, Z. Hadj-Sadok, and J.L. Gouzé. Observers for the biotechnological processes with unknown kinetics, application to wastewater treatment. Proc. on 39th IEEE Conference on Decision and Control, 2000.

$\mathrm{Ph}$. Bogaerts. A hybrid asymptotic-kalman observer for bioprocesses. Bioprocess Engineering, 20:249-255, 1999.

M. J. Brennan, S. Da-Silva, and V. Lopes. Design of a control system using linear matrix inequalities for the active vibration control of a plate. J. of Intelligent Material Systems and Structures, 17:81-93, Jan 2006.

B. Chachuat and O. Bernard. Design of probabilistic observers for massbalanced based bioprocess models. 2002 .

M. Chilali, P. Gahinet, and P. Apkarian. Robust pole placement in lmi regions. IEEE Tran. on Autom. Control, 44(12):2257-2270, Dec. 1999.

H.H. Choi and K.-S. Ro. Lmi-based sliding-mode observer design method. IEEE Proc. Control Theory Appl., 152(1):113-115, Jan. 2005.

A. Chéruy. Software sensors in bioprocess engineering. J. of Biotechnology, 52:193-199, 1997.

D.F. Coutinho, M. Curcio, J. Mladic, and A. Bazanella. Robust observer design for a class of nonlinear systems. Proc. of the 44th IEEE Conf. on 
Decision and Control, and the Europan Control Conf., pages 2640-2645, Dec. 2005

J.L. Crassidis and J.L. Junkins. Optimal Estimation of Dynamic Systems. Chapman \& Hall/CRC, 2004.

J.H. Lee D.G. Robertson and J.B. Rawlings. A moving horizon-based approach for least-squares estimation. AIChE Journal, 42, 1996.

D. Dochain. State observation and adaptive linearizing control for distributed parameter (bio)chemical reactors. Int. J. Adapt. Control Signal Process, 15:633-653, 2001.

D. Dochain. State and parameter estimation in chemical and biochemical processes:a tutorial. J. of Process Control, 13:801-818, 2003.

R. Dondo. Mesurement processing for state estimation and fault identification in batch fermentations. Brazilian Journal of Chemical Engeneering, 21(03), 2004.

M. Farza, H. Hammouri, S. Toman, and K. Busawon. Nonlinear observers for parameter estimation in bioprocesses. Chemical Engineering Science, 52(23):4251-4267, 1997.

B. Friedland. Control System Design-An introduction to state-space methods. McGraw-Hill International Editions, 2nd. edition edition, 1987.

J.P. Gauthier and G. Bornard. Observability for any $u(t)$ of a class of nonlinear systems. IEEE Transactions on Automatic Control, AC-26(4):922-926, 1981.

J.P. Gauthier, H. Hammouri, and S. Othman. A simple observer for nonlinear systems. applications to bioreactors. IEEE Transactions on Automatic Control, 37(6):875-880, 1992.

Hector Valdez-Gonzalez Gonzalo Valdez-Gonzalez and Juan Zolezzi. Método de estimación de estados basado en optimización aplicado a un motor de inducción. Rev. Fac. Ing. - Univ. Tarapacá, 14(01), 2006.

J.L. Gouzé and V. Lemesle. A bounded error observer with adjustable rate for a class of bioreactor models. Proc. of the European Control Conference, ECC 2001, 2001.

J.L. Gouzé, A. Rapaport, and M.Z. Hadj-Sadok. Interval observers for uncertain biological systems. Ecological Modelling, 133:45-56, 2000.

D.W. Gu and F.W. Poon. A robust state observer scheme. IEEE Trans. on Automatic Control, 46(12):1958-1963, 2001.

R.D. Gudi and S.L. Shah. Multirate estimation and monitoring of process variable in a bioreactor. Ecological Modelling, 133:45-56, 1993.

R.D. Gudi, S.L. Shah, and M.R. Gray. Multirate state and parameter estimation in a antibiotic fermentation with delayed measurments. Biotechnology and Bioengineering, 44:1271-1278, 1994.

T.M. Guerra. Linear matrix inequality review. Curso impartido en UPV, May 2006. 
D. Henrion, D. Arzelier, D. Peaucelle, and M. S̆ebek. An lmi condition for robust stability of polinomial matriz polytopes. Automatica, 37(3):461-468, Mar. 2001.

M.A. Henson. Biochemical reactor modeling and control. IEEE Control Systems Magazine, 26(4):54-62, Ago 2006.

W. Hines and D.C. Montgomery. Probabilidad y estadística para ingeniería. CECSA, 2a. edition, 2004.

A. Howell and J.K. Hedrick. Nonlinear observer design via convex optimization. Proc. of the American Control Conf., pages 2088-2093, May 2002.

$\mathrm{X}$. Hulhoven, A. V. Wouwer, and Ph. Bogaerts. Hybrid extended luenbergerasymptotic observer for bioprocess state estimation. Chemical Engineering Science, 61(21):7151-7160, 2006.

A. Johnson. The control of fed-batch fermentation processes-a survey. Automatica, 23(6):691-705, 1987.

O. Kammona, E.G. Chatzi, and C. Kiparissides. Recent developments in hardware sensors for the on-line monitoring of polymerization reactions. Rev. Macromol. Chen. Phys., C39(1):57-134, 1999.

R. King, J. Leifheit, and S. Freyer. Automatic identification of mathematical models of chemical and biochemical reaction systems. CHISA, 2002.

D.J. Kozub and J.F. MacGregor. State estimation for semi-batch polymerization reactors. Chemical Eng. Science, 42(5):1047-1062, 1992.

J. O. Trierweiler L. S.Ferreira, S. M. Marcon and A. R. Secchi. Development of a virtual analyzer software for bioprocesses. AIChe Annual meeting 2003, San Francisco, USA, 1, 2003.

V. Lemesle and J.L. Gouzé. Hybrid bounded error observers for uncertain bioreactor models. Bioprocess Biosystems, 27:311-318, 2005.

Chang-Hua Lien. Robust observer-based control of systems with state perturbations via lmi approach. IEEE Trans. on Automatic Control, 49(8): 1365-1370, 2004.

V. Lubenova, I. Rocha, and E.C. Ferreira. Estimation of multiple biomass growth rates and biomass concentration in a class of bioprocesses. Bioprocess Biosyst. Eng., 25:395-406, 2003.

Radhakrishnan Mahadevan and Francis J. Doyle III. A partial flatness approach to nonlinear moving horizon estimation. Proceeding of the 2004 American Control Conference Boston,Massachusetts June 30 - July 2, 2004.

R. Martínez-Guerra, S. Diop, R. Garrido, and A. Osorio-Mirón. On nonlinear system diagnosis via high-gain observers for bioreactors. Proc. of the IEEE Intern. Conference on Control Applications, pages 726-731, Sep. 5-7 2001a.

R. Martínez-Guerra, R. Garrido, and A. Osorio-Mirón. Parametric and state estimation by means of high-gain nonlinear observers: application to a bioreactor. Proc. of the American Control Conference, pages 3807-3808, Jun. 25-27 2001b. 
M.Soroush and S. Valluri. Nonlinear state estimation in the presence of multiple steady states. Ind. Eng. Chem. Res., 35:2645-2659, 1996.

R.K. Mutha, W.R. Cluett, and A. Penlidis. A new multirate-measurementbased estimator: emulsion copolymerization batch reactor case study. Ind. Eng. Chem. Res., American Chemical Society, 36:1036-1047, 1997.

X.Ñian, J. Wanf, W. Gui, J. Huang, and Z. Huang. A constant gain adaptive observer for speed and resistances identification. Industry Applications Conference, 41st. IAS Annual Meeting, 2:712-718, Oct. 2006.

O. Perez, W. Colmenares, P Vega, and M. Sutil. Desigualdades lineales matriciales en el diseño integrado de procesos.

J. Picó. Comunicación personal. 2002.

A. Rapaport and J.L. Gouzé. Parallelotopic and practical observers for nonlinear uncertain systems. Int. J. Control, 76(3):237-251, 2003.

J. A. Romero. Aportaciones al diseño de experimentos y la estimación de variables en biorreactores. Tesis doctoral, Universidad Politécnica de Valenica, Diciembre 2003.

S.A. Rusell, D.G. Robertson, J.H. Lee, and B.A. Ogunnaike. Model-based quality monitoring of batch and semi-batch process. J. of Process Control, 10:317-332, 2000.

Tor Steinar Schei and Tor Arne Johansen. Nonlinear model based/model predictive control with constraints and with/without nonlinear observer. SINTEF Electronics and Cybernetics, Automatic Control.

C. Scherer and S. Weiland. Disc course on linear matrix inequalities in control -2004/2005. Course, Delft University of Technology and Eindhoven University of Technology, 2004. URL http://www.cs.ele.tue.nl/SWeiland/lmi.html.

K. Schügerl. Progress in monitoring, modeling and control of bioprocesses during the last 20 years. J. of Biotechnology, 85:149-173, 2001.

K. Shimizu. A tutorial review on bioprocess systems engineering. Computers Chem. Eng, 20(6/7):915-941, 1996.

D.E. Simanek. Error analysis (non-calculus). Technical report, Lock Haven University, 1996. URL http://www.lhup.edu/ DESIMANEK/errors.htm.

M. Soroush and S. Tatiraju. Nonlinear state estimation in a polymerization reactor. Ind. Eng. Chem. Res., 36:2679-2690, 1997.

G. Stephanopoulos and K.Y. San. Studies on on-line bioreactor identification. Biotechnology and Bioengineering, XXVI:1176-1218, 1984.

N. Tali-Maamar and J.P. Babary. Influence of the sensor location on the practical observability of distributed parameter bioreactors. Proc. IEEE CONTROL '94, pages 255-260, Mar. 21-24 1994.

K. Tanaka, T. Ikeda, and H.O. Wang. Fuzzy regulators and fuzzy observers: Relaxed stability conditions and lmi-based designs. IEEE Trans. on Fuzzy Systems, 6(2):250-265, May 1998. 
S. Tatiraju, M. Soroush, and R. Mutharasan. Multirate nonlinear state and parameter estimation in a bioreactor. Proc. of the American Control Conference, 1998.

S. Tatiraju, M. Soroush, and R. Mutharasan. Multi-rate nonlinear state and parameter estimation in a bioreactor. Biotechnology and Bioengineering, 63(1):22-32, 1999a.

S. Tatiraju, M. Soroush, and B. A. Ogunnaike. Multirate nonlinear state estimation with application to a polymerization reactor. AIChE Journal, 45(4):769-780, Apr. 1999b.

A. Tornambe. Use of asymptotic observers having high-gains in the state and parameter estimation. Proc. of the 28th. Conference on Decision and Control, pages 1791-1794, Dec. 1989.

D. Del Vecchio and R.M. Murray. Local observability and its application to multiple measurement estimation. IEEE Transactions on Industrial Electronics, 38(6):491-496, 2003.

E. Walter and M. Keiffer. Interval analysis for garanteed nonlinear parameter estimation. 13th. IFAC Symposium on System Identification, 2003.

Y. Wang and E.D. Sontag. Orders of inputoutput diferential equations and state space dimensions.

G. Welch and G. Bishop. An introduction to the kalman filter. Technical Report TR 95-041, University of North Carolina at Chapel Hill-Department of Computer Science, March 112002.

N. Zambare and M. Soroush. Multirate control of a polymerization reactor: a comparative study. Proc. of the American Control Conference, pages 2553-2557, Jun 1999. San Diego, Cal.

N. Zambare, M. Sorush, and M. C. Grady. Multirate nonlinear state estimation in a polymerization reactor: a real-time study. Proc. of the Amreican Control Conference, pages 2701-2706, May 8-10 2002. Anchorage, AK.

A. Zemouche, M. Boutayeb, and G. Iulia-Bara. Observer design for nonlinear systems:an approach based on the differential mean value theorem. Proc. 44 IEEE CDC and ECC 2005, pages 6353-6358, Dec. 2005.

L.A. van der Polb G. van Stratena Z.I.T.A. Soons, J. Shia and A. J. B. van Boxtela. Biomass growth and kla estimation using online and offline measurements. CAB10 (Computer Applications in Biotechnology), Cancun, 2007, 2007. 